\title{
Pd-Catalyzed Intramolecular Cyclization via Direct C-H Addition to Nitriles: Skeletal Diverse Synthesis of Fused Polycyclic Indoles
}

Ting-Ting Wang, Lang Zhao, Yan-Jing Zhang, Wei-Wei Liao*

Department of Organic Chemistry, College of Chemistry, Jilin University, Changchun 130012, China

E-mail:wliao@jlu.edu.cn

Table of Contents

I. General Information

II. Preparation of Substrates

III. Reaction Condition Screening

IV. General Procedure and Experimental Details of Palladium-Catalyzed Intramolecular Cyclization and Proposed Mechanism

V. References

VI. Crystal Data and Structure Refinement

VII. ${ }^{1} \mathrm{H}$ and ${ }^{13} \mathrm{C}$ NMR Spectral Copies 


\section{General Information}

All reactions were carried out under inert atmospheric condition unless otherwise noted, and solvents were dried according to established procedures. Reactions were monitored by thin layer chromatography (TLC) visualizing with ultraviolet light (UV), $\mathrm{KMnO}_{4}$, p-anisaldehyde stain, and phosphomolybdic acid (PMA) stain; column chromatography purifications were carried out using silica gel. Proton nuclear magnetic resonance $\left({ }^{1} \mathrm{H}\right.$ NMR) spectra were recorded on a 300 or $500 \mathrm{MHz}$ spectrometer in $\mathrm{CDCl}_{3}$, and carbon nuclear magnetic resonance $\left({ }^{13} \mathrm{C}\right.$ NMR) spectra were recorded on $125 \mathrm{MHz}$ spectrometer in $\mathrm{CDCl}_{3}$ unless otherwise noted. Chemical shifts for protons are reported in parts per million downfield from tetramethylsilane (TMS) and are referenced to residual protium in the NMR solvent $\left(\mathrm{CHCl}_{3}=\delta 7.26 \mathrm{ppm}\right)$. Chemical shifts for carbon are reported in parts per million downfield from tetramethylsilane (TMS) and are referenced to the carbon resonances of the solvent residual peak $\left(\mathrm{CDCl}_{3}=\delta 77.16 \mathrm{ppm}\right)$. NMR data are presented as follows: chemical shift $(\delta \mathrm{ppm})$, multiplicity $(\mathrm{s}=$ singlet, $\mathrm{d}=$ doublet, $\mathrm{t}=$ triplet, $\mathrm{q}=$ quartet, $\mathrm{m}=$ multiplet, $\mathrm{br}=$ broad$)$, coupling constant in Hertz $(\mathrm{Hz})$, integration. Mass spectra were recorded on the Bruker MicrOTOF Q II.

\section{Preparation of Substrates}

Preparation of substrates 1a-1c, 1f-1k and 11:

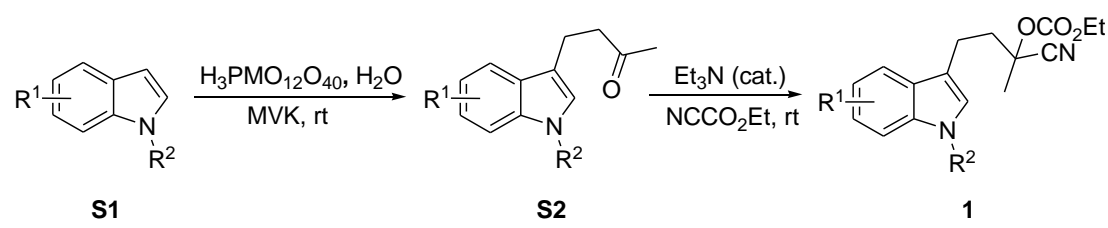

To a solution of $\mathrm{H}_{3} \mathrm{PMO}_{12} \mathrm{O}_{40}(0.55 \mathrm{~mol} \%)$ and $\mathbf{S 1}$ (1.0 equiv.) in water $(0.5 \mathrm{M})$, methyl vinyl ketone (MVK, 1.0 equiv.) was added in one portion. ${ }^{1}$ The mixture was kept at room temperature under vigorous stirring for $1 \mathrm{~h}$. Upon completion, water was added and the aqueous phase was extracted ethyl acetate and dried over $\mathrm{Na}_{2} \mathrm{SO}_{4}$. The solvent was removed under reduced pressure to give crude product, which was purified by column chromatography (silica gel, EtOAc/Petroleum ether) to give $\mathbf{S 2}$.

To a mixture of $\mathbf{S 2}$ (1.0 equiv.) and $\mathrm{Et}_{3} \mathrm{~N}(10 \mathrm{~mol} \%)$, ethyl carbonocyanidate (1.1 equiv.) was slowly added at rt. ${ }^{2}$ Upon completion, the reaction was concentrated in vacuum. The residue was purified by column chromatography (silica gel, EtOAc/Petroleum ether) to give product 1.

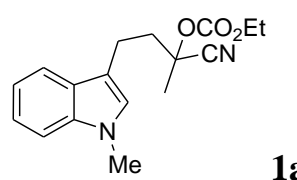

Colorless oil. ${ }^{1} \mathrm{H}$ NMR $\left(300 \mathrm{MHz}, \mathrm{CDCl}_{3}\right)$ d 7.61-7.57 (m, 1H), 7.31-7.28 (m, 1H), 7.26-7.20 (m, 1H), 7.15-7.08 (m, $1 \mathrm{H}), 6.87(\mathrm{~s}, 1 \mathrm{H}), 4.27(\mathrm{q}, J=7.1 \mathrm{~Hz}, 2 \mathrm{H}), 3.74(\mathrm{~s}, 3 \mathrm{H}), 3.11-2.93(\mathrm{~m}, 2 \mathrm{H}), 2.47-2.25(\mathrm{~m}, 2 \mathrm{H}), 1.85(\mathrm{~s}, 3 \mathrm{H}), 1.35$ $(\mathrm{t}, J=7.1 \mathrm{~Hz}, 3 \mathrm{H}) .{ }^{13} \mathrm{C} \mathrm{NMR}\left(125 \mathrm{MHz}, \mathrm{CDCl}_{3}\right) \delta 152.75,137.13,127.45,126.34,121.86,119.00,118.76,118.54$, $112.53,109.42,74.00,64.97,40.64,32.72,24.63,19.83$, 14.25. HRMS (ESI): calcd. for $\mathrm{C}_{17} \mathrm{H}_{21} \mathrm{~N}_{2} \mathrm{O}_{3}\left([\mathrm{M}+\mathrm{H}]^{+}\right)$: 301.1547 , found 301.1549 .

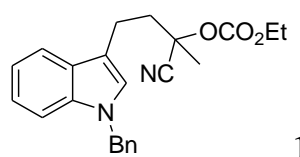


Yellow oil. ${ }^{1} \mathrm{H}$ NMR $\left(500 \mathrm{MHz}, \mathrm{CDCl}_{3}\right) \delta 7.61(\mathrm{~d}, J=7.9 \mathrm{~Hz}, 1 \mathrm{H}), 7.32-7.25(\mathrm{~m}, 4 \mathrm{H}), 7.18(\mathrm{t}, J=7.6 \mathrm{~Hz}, 1 \mathrm{H})$, 7.13-7.10 (m, 3H), $6.93(\mathrm{~s}, 1 \mathrm{H}), 5.26(\mathrm{~s}, 2 \mathrm{H}), 4.26(\mathrm{q}, J=7.1 \mathrm{~Hz}, 2 \mathrm{H}), 3.10-2.96(\mathrm{~m}, 2 \mathrm{H}), 2.46-2.39(\mathrm{~m}, 1 \mathrm{H}), 2.35$ $-2.29(\mathrm{~m}, 1 \mathrm{H}), 1.85(\mathrm{~s}, 3 \mathrm{H}), 1.34(\mathrm{t}, J=7.1 \mathrm{~Hz}, 3 \mathrm{H}) .{ }^{13} \mathrm{C} \mathrm{NMR}\left(125 \mathrm{MHz}, \mathrm{CDCl}_{3}\right) \delta 152.78,137.65,136.91,128.90$, $127.82,127.77,126.98,125.63,122.14,119.35,118.94,118.54,113.40,109.91,74.05,64.98,50.05,40.53,24.70$, 19.93, 14.28. HRMS (ESI): calcd. for $\mathrm{C}_{23} \mathrm{H}_{24} \mathrm{~N}_{2} \mathrm{O}_{3}\left([\mathrm{M}+\mathrm{H}]^{+}\right)$: 377.1860, found 377.1847.

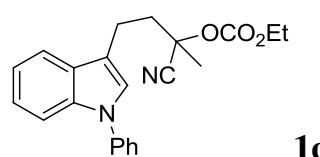

Colourless oil. ${ }^{1} \mathrm{H}$ NMR $\left(500 \mathrm{MHz}, \mathrm{CDCl}_{3}\right) \delta 7.65(\mathrm{~d}, J=7.7 \mathrm{~Hz}, 1 \mathrm{H}), 7.55(\mathrm{~d}, J=8.1 \mathrm{~Hz}, 1 \mathrm{H}), 7.53-7.44(\mathrm{~m}, 4 \mathrm{H})$, $7.33(\mathrm{t}, J=6.9 \mathrm{~Hz}, 1 \mathrm{H}), 7.27-7.15(\mathrm{~m}, 3 \mathrm{H}), 4.27(\mathrm{q}, J=7.1 \mathrm{~Hz}, 2 \mathrm{H}), 3.18-3.01(\mathrm{~m}, 2 \mathrm{H}), 2.54-2.44(\mathrm{~m}, 1 \mathrm{H})$, $2.40-2.34(\mathrm{~m}, 1 \mathrm{H}), 1.88(\mathrm{~s}, 3 \mathrm{H}), 1.35(\mathrm{t}, J=7.1 \mathrm{~Hz}, 3 \mathrm{H}) .{ }^{13} \mathrm{C} \mathrm{NMR}\left(125 \mathrm{MHz}, \mathrm{CDCl}_{3}\right) \delta 152.77,139.79,136.24$, $129.73,128.54,126.40,125.36,124.23,122.81,120.24,119.05,118.51,115.19,110.78,74.00,65.02,40.36,24.71$, 19.86, 14.27. HRMS (ESI): calcd. for $\mathrm{C}_{22} \mathrm{H}_{22} \mathrm{~N}_{2} \mathrm{O}_{3}\left([\mathrm{M}+\mathrm{H}]^{+}\right): 363.1703$ found 363.1708<smiles>CCOC1CCc2c(C)cccc2N1C</smiles>

1f

Yellow oil. ${ }^{1} \mathrm{H}$ NMR $\left(500 \mathrm{MHz}, \mathrm{CDCl}_{3}\right) \delta$ 7.14-7.09 (m, 2H), 6.86-6.84 (m, 2H), $4.28(\mathrm{q}, J=7.1 \mathrm{~Hz}, 2 \mathrm{H}), 3.72(\mathrm{~s}$, $3 \mathrm{H}), 3.26-3.09(\mathrm{~m}, 2 \mathrm{H}), 2.73(\mathrm{~s}, 3 \mathrm{H}), 2.45-2.36(\mathrm{~m}, 1 \mathrm{H}), 2.31-2.25(\mathrm{~m}, 1 \mathrm{H}), 1.87(\mathrm{~s}, 3 \mathrm{H}), 1.36(\mathrm{t}, J=7.1 \mathrm{~Hz}$, $3 \mathrm{H}) .{ }^{13} \mathrm{C}$ NMR $\left(125 \mathrm{MHz}, \mathrm{CDCl}_{3}\right) \delta 152.78,137.58,130.83,126.73,126.07,121.93,120.87,118.52,113.50$, 107.33, 73.90, 65.00, 42.34, 32.84, 24.79, 21.66, 20.40, 14.30. HRMS (ESI): calcd. for $\mathrm{C}_{18} \mathrm{H}_{22} \mathrm{~N}_{2} \mathrm{O}_{3}\left([\mathrm{M}+\mathrm{H}]^{+}\right)$: 315.1703 , found 315.1707 .

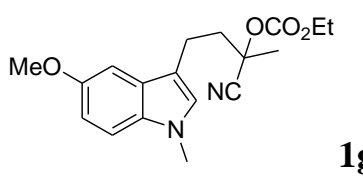

White solid (150 mg, 91\%), mp: 64.2-65.3 ${ }^{\circ} \mathrm{C} .{ }^{1} \mathrm{H}$ NMR (500 MHz, $\left.\mathrm{CDCl}_{3}\right) \delta 7.19(\mathrm{~d}, J=8.8 \mathrm{~Hz}, 1 \mathrm{H}), 7.03(\mathrm{~s}, 1 \mathrm{H})$, 6.91-6.89 (m, 1H), $6.84(\mathrm{~s}, 1 \mathrm{H}), 4.27(\mathrm{q}, J=7.1 \mathrm{~Hz}, 2 \mathrm{H}), 3.88(\mathrm{~s}, 3 \mathrm{H}), 3.71(\mathrm{~s}, 3 \mathrm{H}), 3.08-2.89(\mathrm{~m}, 2 \mathrm{H}), 2.46-$ $2.36(\mathrm{~m}, 1 \mathrm{H}), 2.33-2.27(\mathrm{~m}, 1 \mathrm{H}), 1.86(\mathrm{~s}, 3 \mathrm{H}), 1.35(\mathrm{t}, J=7.1 \mathrm{~Hz}, 3 \mathrm{H}) \cdot{ }^{13} \mathrm{C} \mathrm{NMR}\left(125 \mathrm{MHz}, \mathrm{CDCl}_{3}\right) \delta 153.91$, 152.78, 132.54, 127.74, 126.90, 118.59, 112.09, 112.03, 110.26, 100.59, 74.08, 65.01, 56.13, 40.47, 32.94, 24.68, 19.85, 14.29. HRMS (ESI): calcd. for $\mathrm{C}_{18} \mathrm{H}_{22} \mathrm{~N}_{2} \mathrm{O}_{4}\left([\mathrm{M}+\mathrm{H}]^{+}\right)$: 331.1652 , found 331.1656 .<smiles></smiles>

Yellow oil. ${ }^{1} \mathrm{H}$ NMR $\left(500 \mathrm{MHz}, \mathrm{CDCl}_{3}\right) \delta 7.64(\mathrm{~s}, 1 \mathrm{H}), 7.21(\mathrm{~d}, J=8.3 \mathrm{~Hz}, 1 \mathrm{H}), 7.06(\mathrm{~d}, J=8.6 \mathrm{~Hz}, 1 \mathrm{H}), 6.79$ (s, $1 \mathrm{H}), 4.23(\mathrm{q}, J=7.1 \mathrm{~Hz}, 2 \mathrm{H}), 3.61(\mathrm{~s}, 3 \mathrm{H}), 2.99-2.78(\mathrm{~m}, 2 \mathrm{H}), 2.35-2.27(\mathrm{~m}, 1 \mathrm{H}), 2.27-2.18(\mathrm{~m}, 1 \mathrm{H}), 1.80(\mathrm{~s}$, $3 \mathrm{H}), 1.32(\mathrm{t}, J=7.1 \mathrm{~Hz}, 3 \mathrm{H}) .{ }^{13} \mathrm{C}$ NMR $\left(125 \mathrm{MHz}, \mathrm{CDCl}_{3}\right) \delta 152.72,135.81,129.16,127.59,124.68,121.31$, $118.44,112.45,112.25,110.96,73.84,65.04,40.56,32.93,24.66,19.65,14.27$. HRMS (ESI): calcd. for $\mathrm{C}_{17} \mathrm{H}_{19} \mathrm{BrN}_{2} \mathrm{O}_{3}\left([\mathrm{M}+\mathrm{H}]^{+}\right): 379.0652$, found 379.0658 . 
$1 \mathrm{i}$

Yellow oil. ${ }^{1} \mathrm{H}$ NMR $\left(500 \mathrm{MHz}, \mathrm{CDCl}_{3}\right) \delta 7.53(\mathrm{~s}, 1 \mathrm{H}), 7.21-7.14(\mathrm{~m}, 2 \mathrm{H}), 6.89(\mathrm{~s}, 1 \mathrm{H}), 4.27(\mathrm{q}, J=7.1 \mathrm{~Hz}, 2 \mathrm{H})$, $3.72(\mathrm{~s}, 3 \mathrm{H}), 3.05-2.88(\mathrm{~m}, 2 \mathrm{H}), 2.40-2.34(\mathrm{~m}, 1 \mathrm{H}), 2.30-2.24(\mathrm{~m}, 1 \mathrm{H}), 1.85(\mathrm{~s}, 3 \mathrm{H}), 1.35(\mathrm{t}, J=7.1 \mathrm{~Hz}, 3 \mathrm{H}) .{ }^{13} \mathrm{C}$ $\operatorname{NMR}\left(125 \mathrm{MHz}, \mathrm{CDCl}_{3}\right) \delta 152.74,135.61,128.52,127.75,124.97,122.17,118.45,118.24,112.36,110.51,73.88$, 65.02, 40.58, 32.92, 24.68, 19.68, 14.26. HRMS (ESI): calcd. for $\mathrm{C}_{17} \mathrm{H}_{19} \mathrm{ClN}_{2} \mathrm{O}_{3}\left([\mathrm{M}+\mathrm{H}]^{+}\right)$: 335.1157 , found 335.1163.

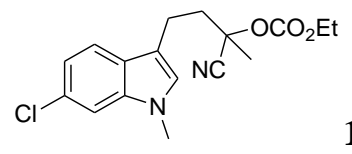

$\mathbf{1 j}$

Yellow oil. ${ }^{1} \mathrm{H}$ NMR $\left(500 \mathrm{MHz}, \mathrm{CDCl}_{3}\right) \delta 7.47(\mathrm{~d}, J=8.4 \mathrm{~Hz}, 1 \mathrm{H}), 7.27(\mathrm{~d}, J=1.5 \mathrm{~Hz}, 1 \mathrm{H}), 7.07$ (dd, $J=8.4,1.6$ $\mathrm{Hz}, 1 \mathrm{H}), 6.85(\mathrm{~s}, 1 \mathrm{H}), 4.27(\mathrm{q}, J=7.1 \mathrm{~Hz}, 2 \mathrm{H}), 3.69(\mathrm{~s}, 3 \mathrm{H}), 3.05-2.91(\mathrm{~m}, 2 \mathrm{H}), 2.41-2.35(\mathrm{~m}, 1 \mathrm{H}), 2.30-2.24(\mathrm{~m}$, $1 \mathrm{H}), 1.85(\mathrm{~s}, 3 \mathrm{H}), 1.35(\mathrm{t}, J=7.1 \mathrm{~Hz}, 3 \mathrm{H}) .{ }^{13} \mathrm{C} \mathrm{NMR}\left(125 \mathrm{MHz}, \mathrm{CDCl}_{3}\right) \delta 152.72,137.55,127.99,127.01,126.08$, 119.70, 119.68, 118.47, 112.90, 109.48, 73.92, 65.02, 40.55, 32.82, 24.66, 19.74, 14.26. HRMS (ESI): calcd. for $\mathrm{C}_{17} \mathrm{H}_{19} \mathrm{ClN}_{2} \mathrm{O}_{3}\left([\mathrm{M}+\mathrm{H}]^{+}\right): 335.1157$, found 335.1161 .

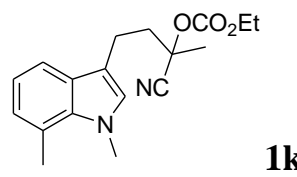

Yellow oil. ${ }^{1} \mathrm{H}$ NMR $\left(500 \mathrm{MHz}, \mathrm{CDCl}_{3}\right) \delta$ 7.44-7.42 (m, 1H), $7.04-6.96(\mathrm{~m}, 1 \mathrm{H}), 6.93(\mathrm{~d}, J=5.6 \mathrm{~Hz}, 1 \mathrm{H}), 6.78(\mathrm{~s}$, $1 \mathrm{H}), 4.34-4.25(\mathrm{~m}, 2 \mathrm{H}), 4.02(\mathrm{~s}, 3 \mathrm{H}), 3.09-2.90(\mathrm{~m}, 2 \mathrm{H}), 2.77(\mathrm{~s}, 3 \mathrm{H}), 2.46-2.35(\mathrm{~m}, 1 \mathrm{H}), 2.35-2.26(\mathrm{~m}, 1 \mathrm{H})$, $1.91-1.84(\mathrm{~m}, 3 \mathrm{H}), 1.42-1.34(\mathrm{~m}, 3 \mathrm{H}) .{ }^{13} \mathrm{C} \mathrm{NMR}\left(125 \mathrm{MHz}, \mathrm{CDCl}_{3}\right) \delta 152.77,135.88,128.55,128.03,124.54$, 121.52, 119.38, 118.56, 116.83, 112.25, 74.03, 64.98, 40.60, 36.65, 24.66, 19.82, 19.74, 14.28. HRMS (ESI): calcd. for $\mathrm{C}_{18} \mathrm{H}_{22} \mathrm{~N}_{2} \mathrm{O}_{3}\left([\mathrm{M}+\mathrm{H}]^{+}\right): 315.1703$, found 315.1709.

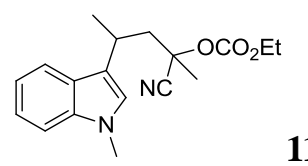

\section{1}

Yellow oil ( $\mathrm{dr}=1: 1$, unseparated diastereoisomers). The data for the diastereoisomers: ${ }^{1} \mathrm{H}$ NMR $(500 \mathrm{MHz}$, $\left.\mathrm{CDCl}_{3}\right) \delta 7.65(\mathrm{~d}, J=7.5 \mathrm{~Hz}, 1 \mathrm{H}), 7.64(\mathrm{~d}, J=8.0 \mathrm{~Hz}, 1 \mathrm{H}), 7.28(\mathrm{~d}, J=8.2 \mathrm{~Hz}, 2 \mathrm{H}), 7.24-7.20(\mathrm{~m}, 2 \mathrm{H}), 7.12-7.08$ $(\mathrm{m}, 2 \mathrm{H}), 6.87(\mathrm{~s}, 2 \mathrm{H}), 4.22-4.07(\mathrm{~m}, 4 \mathrm{H}), 3.73(\mathrm{~s}, 6 \mathrm{H}), 3.49-3.38(\mathrm{~m}, 2 \mathrm{H}), 2.61(\mathrm{dd}, J=14.6,6.3 \mathrm{~Hz}, 1 \mathrm{H}), 2.50$ $(\mathrm{dd}, J=14.3,7.1 \mathrm{~Hz}, 1 \mathrm{H}), 2.39-2.27(\mathrm{~m}, 2 \mathrm{H}), 1.76(\mathrm{~s}, 3 \mathrm{H}), 1.64(\mathrm{~s}, 3 \mathrm{H}), 1.50(\mathrm{dd}, J=6.9,4.6 \mathrm{~Hz}, 3 \mathrm{H}), 1.28(\mathrm{t}, J=$ $7.1 \mathrm{~Hz}, 3 \mathrm{H}) .{ }^{13} \mathrm{C}$ NMR $\left(125 \mathrm{MHz}, \mathrm{CDCl}_{3}\right) \delta 152.62,152.54,137.43,137.32,126.53,126.39,125.72,125.45$, $121.80,121.77,119.56,119.45,119.27,119.08,119.01,118.95,118.91,118.54,109.54,109.48,74.49,73.74$, 64.76, 46.55, 46.30, 32.76, 28.03, 26.93, 25.29, 22.83, 22.53, 14.22. HRMS (ESI): calcd. for $\mathrm{C}_{18} \mathrm{H}_{22} \mathrm{~N}_{2} \mathrm{O}_{3}\left([\mathrm{M}+\mathrm{H}]^{+}\right)$: 315.1703 found 315.1711 .

\section{Preparation of 1e:}




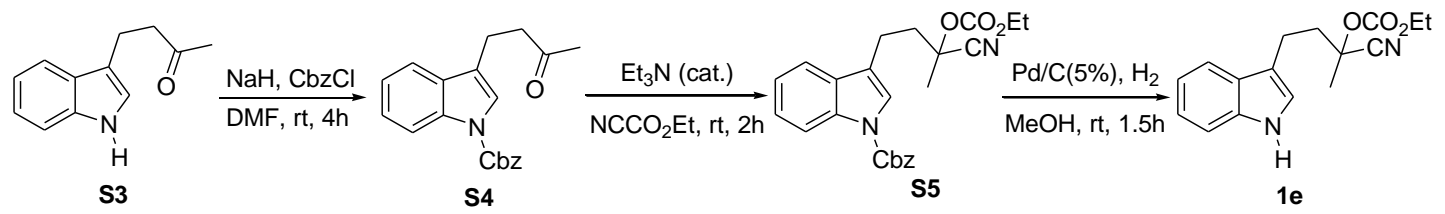

To a solution of $\mathbf{S 3}^{1}$ (1.0 equiv.) in dry DMF ( $\left.0.5 \mathrm{M}\right)$ was added NaH (1.2 equiv., $60 \%$ suspension in mineral oil) under argon at $0{ }^{\circ} \mathrm{C}$. After $30 \mathrm{~min}$, phenyl chloroformate (614 mg, 1.2 equiv.) was added and the mixture was stirred at rt. After $2 \mathrm{~h}$, water was added and the aqueous phase was extracted with EtOAc. The combined organic layers were dried over $\mathrm{Na}_{2} \mathrm{SO}_{4}$, filtered and the solvent was removed under reduced pressure. The residue was purified by column chromatography to afford $\mathbf{S 4}$.

Compound $\mathbf{S 5}$ was prepared from compound $\mathbf{S 4}$ according to the procedure for the synthesis of compound $\mathbf{1 a}$.

To a solution of $\mathbf{S 5}$ (1.0 equiv.) in $\mathrm{MeOH}(0.3 \mathrm{M})$, palladium on carbon $(5 \% \mathrm{Pd})$ was added. ${ }^{3}$ The reaction was stirred for 2 hours under a $\mathrm{H}_{2}$ atmosphere at rt. Then the reaction was diluted with $\mathrm{CH}_{2} \mathrm{Cl}_{2}$ and filtered through a short Celite ${ }^{\circledR}$ pad, and the filtrate was concentrated under reduced pressure. The residue was purified by column chromatography (silica gel, EtOAc/Petroleum ether) to give 1e.

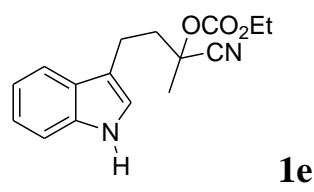

White solid, mp: $65.8-66 .{ }^{\circ} \mathrm{C} .{ }^{1} \mathrm{H}$ NMR $\left(500 \mathrm{MHz}, \mathrm{CDCl}_{3}\right) \delta 8.00(\mathrm{~s}, 1 \mathrm{H}), 7.61(\mathrm{~d}, J=7.9 \mathrm{~Hz}, 1 \mathrm{H}), 7.37(\mathrm{~d}, J=$ $8.1 \mathrm{~Hz}, 1 \mathrm{H}), 7.21(\mathrm{t}, J=7.2 \mathrm{~Hz}, 1 \mathrm{H}), 7.14(\mathrm{t}, J=7.8 \mathrm{~Hz}, 1 \mathrm{H}), 7.03(\mathrm{~s}, 1 \mathrm{H}), 4.27$ (q, $J=7.1 \mathrm{~Hz}, 2 \mathrm{H}), 3.14-2.96(\mathrm{~m}$, $2 \mathrm{H}), 2.48-2.40(\mathrm{~m}, 1 \mathrm{H}), 2.36-2.30(\mathrm{~m}, 1 \mathrm{H}), 1.87(\mathrm{~s}, 1 \mathrm{H}), 1.35(\mathrm{t}, J=7.2 \mathrm{~Hz}, 3 \mathrm{H}) .{ }^{13} \mathrm{C}$ NMR $\left(125 \mathrm{MHz}, \mathrm{CDCl}_{3}\right) \delta$ 152.77, 136.40, 127.05, 122.29, 121.55, 119.53, 118.64, 118.57, 114.00, 111.38, 74.01, 65.01, 40.35, 24.62, 19.91, 14.25. HRMS (ESI): calcd. for $\mathrm{C}_{16} \mathrm{H}_{18} \mathrm{~N}_{2} \mathrm{O}_{3}\left([\mathrm{M}+\mathrm{H}]^{+}\right)$: 287.1390, found 287.1400.

\section{Preparation of 11:}

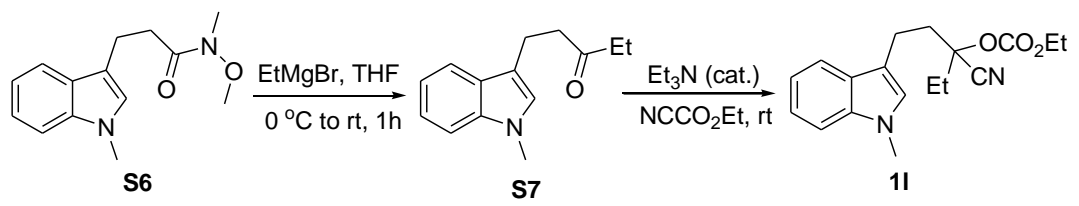

In a nitrogen atmosphere, to a solution of $\mathbf{S 6}^{4}$ (1.0 equiv.) in dry $\operatorname{THF}(0.2 M)$ was added $\operatorname{EtMgBr}(1.1$ equiv., 1.1 $M$ in THF) dropwise at $0{ }^{\circ} \mathrm{C}$. Then, the reaction mixture was stirred at $\mathrm{rt}$ for $2 \mathrm{~h}$. After completion, the reaction was quenched with saturated ammonium chloride solution at $0{ }^{\circ} \mathrm{C}$ and the mixture was extracted with DCM. The combined organic phases were washed with brine before being dried over $\mathrm{Na}_{2} \mathrm{SO}_{4}$ and concentrated in vacuo. The residue was purified by column chromatography (silica gel, EtOAc/Petroleum ether) to give $\mathbf{S} 7$.

Compound $\mathbf{1 l}$ was prepared from compound $\mathbf{S 7}$ according to the procedure for the synthesis of compound $\mathbf{1 a}$.<smiles>CCOC(=O)CCc1cn(C)c2ccccc12</smiles>

Colorless oil. ${ }^{1} \mathrm{H}$ NMR $\left(500 \mathrm{MHz}, \mathrm{CDCl}_{3}\right) \delta 7.58(\mathrm{~d}, J=7.9 \mathrm{~Hz}, 1 \mathrm{H}), 7.28(\mathrm{~d}, J=8.2 \mathrm{~Hz}, 1 \mathrm{H}), 7.22(\mathrm{t}, J=7.5 \mathrm{~Hz}$, $1 \mathrm{H}), 7.11(\mathrm{t}, J=7.4 \mathrm{~Hz}, 1 \mathrm{H}), 6.86(\mathrm{~s}, 1 \mathrm{H}), 4.24(\mathrm{q}, J=7.1 \mathrm{~Hz}, 2 \mathrm{H}), 3.73(\mathrm{~s}, 3 \mathrm{H}), 3.06-2.88(\mathrm{~m}, 2 \mathrm{H}), 2.50-2.32$ 
$(\mathrm{m}, 2 \mathrm{H}), 2.28-2.07(\mathrm{~m}, 2 \mathrm{H}), 1.33(\mathrm{t}, J=7.1 \mathrm{~Hz}, 3 \mathrm{H}), 1.12(\mathrm{t}, J=7.4 \mathrm{~Hz}, 3 \mathrm{H}) \cdot{ }^{13} \mathrm{C}$ NMR $\left(125 \mathrm{MHz}, \mathrm{CDCl}_{3}\right) \delta$ $152.73,137.18,127.49,126.34,121.87,119.03,118.76,117.97,112.68,109.41,78.27,64.90,37.16,32.70,30.09$, 19.62, 14.25, 8.21. HRMS (ESI): calcd. for $\mathrm{C}_{18} \mathrm{H}_{22} \mathrm{~N}_{2} \mathrm{O}_{3}\left([\mathrm{M}+\mathrm{H}]^{+}\right): 315.1703$, found 315.1714.

\section{Preparation of 1m-10:}

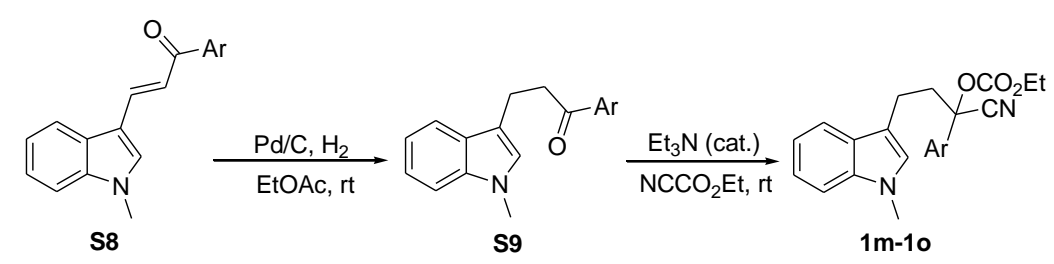

The $\mathbf{S 8}^{5}$ (1.0 equiv.) was dissolved in EtOAc $(0.2 \mathrm{M})$, palladium on carbon (5\% Pd) was added. The reaction was stirred for 1.5 hours under a $\mathrm{H}_{2}$ atmosphere. Then the reaction was diluted with $\mathrm{CH}_{2} \mathrm{Cl}_{2}$ and filtered through a short Celite ${ }^{\circledR}$ pad, and the filtrate was concentrated under reduced pressure. The residue was purified by column chromatography (silica gel, EtOAc/Petroleum ether) to give S9.

Compounds 1m-1o were prepared from S9 according to the procedure for the synthesis of compound 1a.<smiles>CCOC1(OCC)CCc2c(n(C)c3ccccc23)N1</smiles>

Colorless oil. ${ }^{1} \mathrm{H}$ NMR $\left(500 \mathrm{MHz}, \mathrm{CDCl}_{3}\right) \delta 7.58(\mathrm{~d}, J=7.2 \mathrm{~Hz}, 2 \mathrm{H}), 7.47(\mathrm{~d}, J=7.9 \mathrm{~Hz}, 1 \mathrm{H}), 7.43-7.36(\mathrm{~m}, 3 \mathrm{H})$, $7.24(\mathrm{~d}, J=8.2 \mathrm{~Hz}, 1 \mathrm{H}), 7.20-7.17(\mathrm{~m}, 1 \mathrm{H}), 7.07$ (t, $J=7.4 \mathrm{~Hz}, 1 \mathrm{H}), 6.77(\mathrm{~s}, 1 \mathrm{H}), 4.27-4.07$ (m, 2H), $3.67(\mathrm{~s}, 3 \mathrm{H})$, 3.10-3.04 (m, 1H), $2.88-2.76(\mathrm{~m}, 1 \mathrm{H}), 2.68-2.55(\mathrm{~m}, 1 \mathrm{H}), 2.50-2.37(\mathrm{~m}, 1 \mathrm{H}), 1.27(\mathrm{t}, J=7.1 \mathrm{~Hz}, 3 \mathrm{H}) .{ }^{13} \mathrm{C}$ $\operatorname{NMR}\left(125 \mathrm{MHz}, \mathrm{CDCl}_{3}\right) \delta 152.43,137.11,136.94,129.49,129.09,127.44,126.28,124.88,121.80,118.94,118.69$, 117.30, 112.41, 109.34, 79.35, 65.15, 43.55, 32.61, 20.14, 14.16. HRMS (ESI): calcd. for $\mathrm{C}_{22} \mathrm{H}_{22} \mathrm{~N}_{2} \mathrm{O}_{3}\left([\mathrm{M}+\mathrm{H}]^{+}\right)$: 363.1703 , found 363.1698 .<smiles></smiles>

Yellow oil. ${ }^{1} \mathrm{H}$ NMR $\left(500 \mathrm{MHz}, \mathrm{CDCl}_{3}\right) \delta 7.62-7.34(\mathrm{~m}, 5 \mathrm{H}), 7.32-7.18(\mathrm{~m}, 2 \mathrm{H}), 7.09$ (t, $\left.J=6.9 \mathrm{~Hz}, 1 \mathrm{H}\right), 6.80$ (s, 1H), 4.27-4.13 (m, 2H), $3.71(\mathrm{~s}, 3 \mathrm{H}), 3.07(\mathrm{t}, J=11.4 \mathrm{~Hz}, 1 \mathrm{H}), 2.82(\mathrm{t}, J=11.1 \mathrm{~Hz}, 1 \mathrm{H}), 2.62(\mathrm{t}, J=10.9 \mathrm{~Hz}$, $1 \mathrm{H}), 2.42(\mathrm{t}, J=11.0 \mathrm{~Hz}, 1 \mathrm{H}), 1.30(\mathrm{t}, J=6.8 \mathrm{~Hz}, 3 \mathrm{H}) .{ }^{13} \mathrm{C} \mathrm{NMR}\left(125 \mathrm{MHz}, \mathrm{CDCl}_{3}\right) \delta 152.40,137.09,135.63$, 135.50, 129.37, 127.36, 126.47, 126.37, 121.90, 119.03, 118.68, 116.97, 112.15, 109.44, 78.78, 65.40, 43.37, 32.74, 20.17, 14.22. HRMS (ESI): calcd. for $\mathrm{C}_{22} \mathrm{H}_{21} \mathrm{ClN}_{2} \mathrm{O}_{3}\left([\mathrm{M}+\mathrm{H}]^{+}\right)$: 397.1313, found 397.1312.

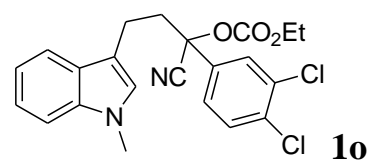

Yellow oil. ${ }^{1} \mathrm{H}$ NMR $\left(500 \mathrm{MHz}, \mathrm{CDCl}_{3}\right) \delta 7.64(\mathrm{~d}, J=2.2 \mathrm{~Hz}, 1 \mathrm{H}), 7.50-7.45(\mathrm{~m}, 2 \mathrm{H}), 7.40(\mathrm{dd}, J=8.5,2.2 \mathrm{~Hz}$, 1H), $7.28-7.24(\mathrm{~m}, 1 \mathrm{H}), 7.21(\mathrm{t}, J=7.6 \mathrm{~Hz}, 1 \mathrm{H}), 7.13-7.06(\mathrm{~m}, 1 \mathrm{H}), 6.80(\mathrm{~s}, 1 \mathrm{H}), 4.28-4.15(\mathrm{~m}, 2 \mathrm{H}), 3.71$ (s, $3 \mathrm{H}), 3.13-3.03(\mathrm{~m}, 1 \mathrm{H}), 2.90-2.81(\mathrm{~m}, 1 \mathrm{H}), 2.64-2.55(\mathrm{~m}, 1 \mathrm{H}), 2.48-2.38(\mathrm{~m}, 1 \mathrm{H}), 1.31(\mathrm{t}, J=7.2 \mathrm{~Hz}, 3 \mathrm{H})$. ${ }^{13} \mathrm{C}$ NMR $\left(125 \mathrm{MHz}, \mathrm{CDCl}_{3}\right) \delta 152.33,137.12,133.97,133.57,131.06,127.30,127.18,126.45,124.44,121.96$, $119.10,118.65,116.54,111.89,109.46,78.20,65.59,43.18,32.74,20.22,14.21$. HRMS (ESI): calcd. for 
$\mathrm{C}_{22} \mathrm{H}_{20} \mathrm{Cl}_{2} \mathrm{~N}_{2} \mathrm{O}_{3}\left([\mathrm{M}+\mathrm{H}]^{+}\right): 431.0924$, found 431.0919.

Compounds 1q-1s were prepared according to the procedure for the synthesis of compounds 1m-1n. Compound 1t was prepared according to the procedure for the synthesis of compound $\mathbf{1 e .}$

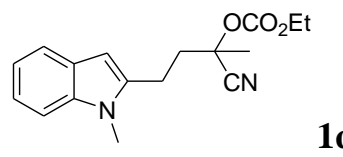

$1 q$

Yellow oil. ${ }^{1} \mathrm{H}$ NMR $\left(500 \mathrm{MHz}, \mathrm{CDCl}_{3}\right) \delta 7.52(\mathrm{~d}, J=7.8 \mathrm{~Hz}, 1 \mathrm{H}), 7.25(\mathrm{~d}, J=8.2 \mathrm{~Hz}, 1 \mathrm{H}), 7.17(\mathrm{t}, J=7.6 \mathrm{~Hz}$, $1 \mathrm{H}), 7.07(\mathrm{t}, J=7.4 \mathrm{~Hz}, 1 \mathrm{H}), 6.25(\mathrm{~s}, 1 \mathrm{H}), 4.25(\mathrm{q}, J=7.1 \mathrm{~Hz}, 2 \mathrm{H}), 3.64(\mathrm{~s}, 3 \mathrm{H}), 3.07-2.93(\mathrm{~m}, 2 \mathrm{H}), 2.45-2.39(\mathrm{~m}$, 1H), 2.34-2.28 (m, 1H), $1.86(\mathrm{~s}, 3 \mathrm{H}), 1.33(\mathrm{t}, J=7.1 \mathrm{~Hz}, 3 \mathrm{H}) .{ }^{13} \mathrm{C} \mathrm{NMR}\left(125 \mathrm{MHz}, \mathrm{CDCl}_{3}\right) \delta 152.58,138.12$, 137.46, 127.64, 121.12, 120.00, 119.53, 118.19, 108.94, 99.05, 73.52, 65.08, 38.75, 29.52, 24.62, 21.54, 14.17. HRMS (ESI): calcd. for $\mathrm{C}_{17} \mathrm{H}_{20} \mathrm{~N}_{2} \mathrm{O}_{3}\left([\mathrm{M}+\mathrm{H}]^{+}\right): 301.1547$, found 301.1540 .

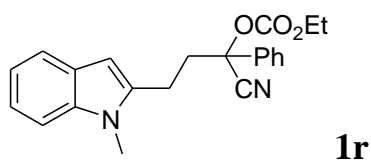

Yellow oil. ${ }^{1} \mathrm{H}$ NMR $\left(500 \mathrm{MHz}, \mathrm{CDCl}_{3}\right) \delta 7.61-7.58(\mathrm{~m}, 2 \mathrm{H}), 7.52-7.41(\mathrm{~m}, 4 \mathrm{H}), 7.27-7.24(\mathrm{~m}, 1 \mathrm{H}), 7.16(\mathrm{t}, J=$ $7.6 \mathrm{~Hz}, 1 \mathrm{H}), 7.06(\mathrm{t}, J=7.4 \mathrm{~Hz}, 1 \mathrm{H}), 6.22(\mathrm{~s}, 1 \mathrm{H}), 4.26-4.15(\mathrm{~m}, 2 \mathrm{H}), 3.63(\mathrm{~s}, 3 \mathrm{H}), 3.15-3.06(\mathrm{~m}, 1 \mathrm{H}), 2.89-$ $2.81(\mathrm{~m}, 1 \mathrm{H}), 2.74-2.65(\mathrm{~m}, 1 \mathrm{H}), 2.52-2.44(\mathrm{~m}, 1 \mathrm{H}), 1.30(\mathrm{t}, J=7.1 \mathrm{~Hz}, 3 \mathrm{H}) .{ }^{13} \mathrm{C} \mathrm{NMR}\left(125 \mathrm{MHz}, \mathrm{CDCl}_{3}\right) \delta$ $152.32,138.03,137.51,136.58,129.84,129.33,127.70,124.85,121.20,120.06,119.60,117.08,108.97,99.12$, 78.96, 65.40, 41.70, 29.57, 22.01, 14.20. HRMS (ESI): calcd. for $\mathrm{C}_{22} \mathrm{H}_{22} \mathrm{~N}_{2} \mathrm{O}_{3}\left([\mathrm{M}+\mathrm{H}]^{+}\right): 363.1703$, found 363.1699.

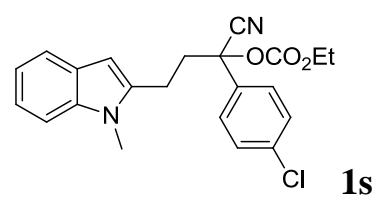

Yellow oil. ${ }^{1} \mathrm{H}$ NMR $\left(500 \mathrm{MHz}, \mathrm{CDCl}_{3}\right) \delta$ 7.59-7.46 (m, 3H), $7.46-7.40(\mathrm{~m}, 2 \mathrm{H}), 7.24(\mathrm{~s}, 1 \mathrm{H}), 7.16(\mathrm{t}, J=7.6 \mathrm{~Hz}$, $1 \mathrm{H}), 7.06(\mathrm{t}, J=7.4 \mathrm{~Hz}, 1 \mathrm{H}), 6.21(\mathrm{~s}, 1 \mathrm{H}), 4.34-4.12(\mathrm{~m}, 2 \mathrm{H}), 3.63(\mathrm{~s}, 3 \mathrm{H}), 3.17-3.01(\mathrm{~m}, 1 \mathrm{H}), 2.91-2.75(\mathrm{~m}$, $1 \mathrm{H}), 2.74-2.61(\mathrm{~m}, 1 \mathrm{H}), 2.50-2.40(\mathrm{~m}, 1 \mathrm{H}), 1.30(\mathrm{t}, J=7.1 \mathrm{~Hz}, 3 \mathrm{H}) .{ }^{13} \mathrm{C} \mathrm{NMR}\left(125 \mathrm{MHz}, \mathrm{CDCl}_{3}\right) \delta 152.24$, 137.73, 137.52, 135.96, 135.15, 129.59, 127.66, 126.40, 121.28, 120.08, 119.65, 116.72, 108.98, 99.18, 78.36, 65.56, 41.50, 29.59, 21.97, 14.19. HRMS (ESI): calcd. for $\mathrm{C}_{21} \mathrm{H}_{19} \mathrm{ClN}_{2} \mathrm{O}_{3}\left([\mathrm{M}+\mathrm{H}]^{+}\right)$: 397.1313, found 397.1318.

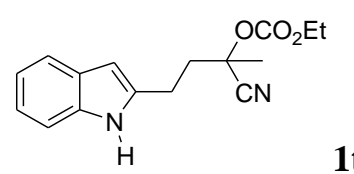

Colourless oil. ${ }^{1} \mathrm{H}$ NMR (500 MHz, CDCl3) $\delta 7.97$ (brs, $\left.1 \mathrm{H}\right), 7.53(\mathrm{~d}, J=7.7 \mathrm{~Hz}, 1 \mathrm{H}), 7.31(\mathrm{~d}, J=8.1 \mathrm{~Hz}, 1 \mathrm{H})$, $7.14(\mathrm{t}, J=7.6 \mathrm{~Hz}, 1 \mathrm{H}), 7.08(\mathrm{t}, J=7.4 \mathrm{~Hz}, 1 \mathrm{H}), 6.28(\mathrm{~s}, 1 \mathrm{H}), 4.26(\mathrm{q}, J=7.1 \mathrm{~Hz}, 2 \mathrm{H}), 3.14-2.96(\mathrm{~m}, 2 \mathrm{H}), 2.50-$ $2.41(\mathrm{~m}, 1 \mathrm{H}), 2.40-2.32(\mathrm{~m}, 1 \mathrm{H}), 1.86(\mathrm{~s}, 3 \mathrm{H}), 1.33(\mathrm{t}, J=7.1 \mathrm{~Hz}, 3 \mathrm{H}) .{ }^{13} \mathrm{C} \mathrm{NMR}\left(125 \mathrm{MHz}, \mathrm{CDCl}_{3}\right) \delta 152.62$, 136.79, 136.12, 128.66, 121.59, 120.06, 119.93, 118.27, 110.65, 100.12, 73.64, 65.16, 39.38, 24.65, 23.02, 14.18. HRMS (ESI): calcd. for $\mathrm{C}_{16} \mathrm{H}_{18} \mathrm{~N}_{2} \mathrm{O}_{3}\left([\mathrm{M}+\mathrm{H}]^{+}\right): 287.1390$, found 287.1385 . 


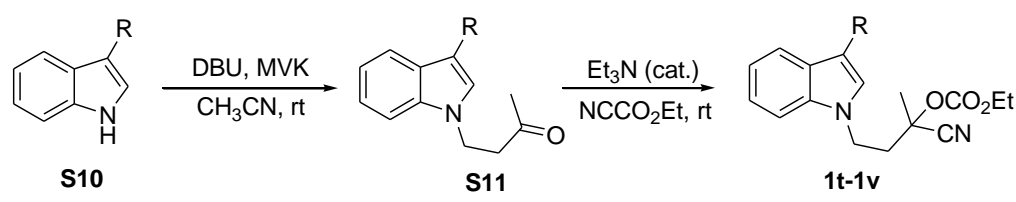

To a solution of $\mathbf{S 1 0}$ (1.0 equiv.) and methyl vinyl ketone (1.5 equiv., $\mathrm{MVK}$ ) in $\mathrm{CH}_{3} \mathrm{CN}$ (2 M) was added DBU (50 mol\%) at rt. Upon completion, the mixture was concentrated under reduced pressure. The residue was purified by column chromatography (silica gel, EtOAc/Petroleum ether) to afford S11. ${ }^{6}$

Compounds $\mathbf{1 t}-\mathbf{1 v}$ were prepared according to the procedure for the synthesis of compound $\mathbf{1 a}$ from $\mathbf{S 2}$.

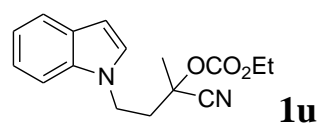

Colorless oil. ${ }^{1} \mathrm{H}$ NMR $\left(500 \mathrm{MHz}, \mathrm{CDCl}_{3}\right) \delta 7.67-7.60(\mathrm{~m}, 1 \mathrm{H}), 7.38(\mathrm{~d}, J=8.1 \mathrm{~Hz}, 1 \mathrm{H}), 7.28-7.21(\mathrm{~m}, 1 \mathrm{H})$, $7.17-7.12(\mathrm{~m}, 1 \mathrm{H}), 7.10(\mathrm{~d}, J=3.0 \mathrm{~Hz}, 1 \mathrm{H}), 6.53(\mathrm{~d}, J=3.0 \mathrm{~Hz}, 1 \mathrm{H}), 4.50-4.36(\mathrm{~m}, 2 \mathrm{H}), 4.31-4.24(\mathrm{~m}, 2 \mathrm{H})$, $2.55-2.47(\mathrm{~m}, 1 \mathrm{H}), 2.46-2.38(\mathrm{~m}, 1 \mathrm{H}), 1.78(\mathrm{~s}, 3 \mathrm{H}), 1.41-1.32(\mathrm{~m}, 3 \mathrm{H}) .{ }^{13} \mathrm{C} \mathrm{NMR}\left(125 \mathrm{MHz}, \mathrm{CDCl}_{3}\right) \delta 152.49$, 135.72, 128.91, 127.58, 122.06, 121.33, 119.85, 117.86, 109.12, 102.21, 72.33, 65.26, 41.54, 39.87, 24.72, 14.22. HRMS (ESI): calcd. for $\mathrm{C}_{16} \mathrm{H}_{18} \mathrm{~N}_{2} \mathrm{O}_{3}\left([\mathrm{M}+\mathrm{H}]^{+}\right): 287.1390$, found 287.1391 .

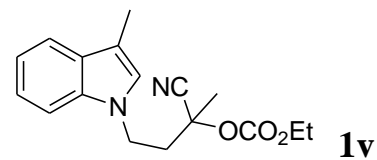

Yellow oil. ${ }^{1} \mathrm{H}$ NMR $\left(500 \mathrm{MHz}, \mathrm{CDCl}_{3}\right) \delta 7.55(\mathrm{~d}, J=7.7 \mathrm{~Hz}, 1 \mathrm{H}), 7.29(\mathrm{~d}, J=8.1 \mathrm{~Hz}, 1 \mathrm{H}), 7.21(\mathrm{t}, J=7.5 \mathrm{~Hz}$, $1 \mathrm{H}), 7.10(\mathrm{t}, J=7.3 \mathrm{~Hz}, 1 \mathrm{H}), 6.82(\mathrm{~s}, 1 \mathrm{H}), 4.36-4.28(\mathrm{~m}, 2 \mathrm{H}), 4.23(\mathrm{q}, J=7.1 \mathrm{~Hz}, 2 \mathrm{H}), 2.47-2.37(\mathrm{~m}, 1 \mathrm{H})$, 2.36-2.31 (m, 1H), $2.30(\mathrm{~s}, 3 \mathrm{H}), 1.72(\mathrm{~s}, 3 \mathrm{H}), 1.33(\mathrm{t}, J=7.1 \mathrm{~Hz}, 3 \mathrm{H}) .{ }^{13} \mathrm{C}$ NMR $\left(125 \mathrm{MHz}, \mathrm{CDCl}_{3}\right) \delta 152.43$, 135.93, 129.04, 125.13, 121.88, 119.28, 119.03, 117.86, 111.22, 108.88, 72.28, 65.15, 41.10, 39.76, 24.56, 14.15, 9.60. HRMS (ESI): calcd. for $\mathrm{C}_{17} \mathrm{H}_{20} \mathrm{~N}_{2} \mathrm{O}_{3}\left([\mathrm{M}+\mathrm{H}]^{+}\right)$: 301.1547, found 301.1558 .

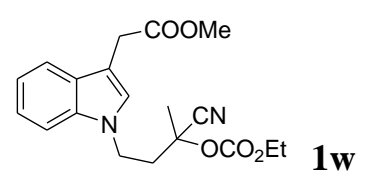

Yellow oil. ${ }^{1} \mathrm{H}$ NMR $\left(500 \mathrm{MHz}, \mathrm{CDCl}_{3}\right) \delta 7.60(\mathrm{~d}, J=7.9 \mathrm{~Hz}, 1 \mathrm{H}), 7.35(\mathrm{~d}, J=8.2 \mathrm{~Hz}, 1 \mathrm{H}), 7.27-7.22(\mathrm{~m}, 1 \mathrm{H})$, $7.14(\mathrm{t}, J=7.4 \mathrm{~Hz}, 1 \mathrm{H}), 7.09(\mathrm{~s}, 1 \mathrm{H}), 4.42-4.37(\mathrm{~m}, 2 \mathrm{H}), 4.27$ (q, $J=7.1 \mathrm{~Hz}, 2 \mathrm{H}), 3.76(\mathrm{~s}, 2 \mathrm{H}), 3.70(\mathrm{~s}, 3 \mathrm{H})$, 2.54-2.45 (m, 1H), $2.44-2.37(\mathrm{~m}, 1 \mathrm{H}), 1.79(\mathrm{~s}, 3 \mathrm{H}), 1.36(\mathrm{t}, J=7.1 \mathrm{~Hz}, 3 \mathrm{H}) .{ }^{13} \mathrm{C} \mathrm{NMR}\left(125 \mathrm{MHz}, \mathrm{CDCl}_{3}\right) \delta$ $172.46,152.50,135.92,128.10,126.45,122.38,119.79,119.42,117.84,109.22,108.06,72.25,65.30,52.12,41.44$, 39.98, 31.09, 24.74, 14.24. HRMS (ESI): calcd. for $\left([\mathrm{M}+\mathrm{H}]^{+}\right)$: 359.1601, found 359.1603.

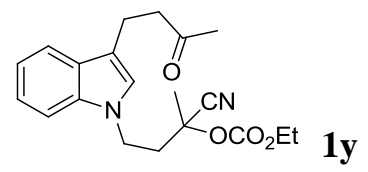

Colourless oil. ${ }^{1} \mathrm{H}$ NMR $\left(500 \mathrm{MHz}, \mathrm{CDCl}_{3}\right) \delta 7.57(\mathrm{~d}, J=7.8 \mathrm{~Hz}, 1 \mathrm{H}), 7.32(\mathrm{~d}, J=8.1 \mathrm{~Hz}, 1 \mathrm{H}), 7.27-7.20(\mathrm{~m}$, $1 \mathrm{H}), 7.12(\mathrm{t}, J=7.4 \mathrm{~Hz}, 1 \mathrm{H}), 6.88(\mathrm{~s}, 1 \mathrm{H}), 4.41-4.31(\mathrm{~m}, 2 \mathrm{H}), 4.26(\mathrm{q}, J=7.1 \mathrm{~Hz}, 2 \mathrm{H}), 3.02(\mathrm{t}, J=7.3 \mathrm{~Hz}, 2 \mathrm{H})$, $2.82(\mathrm{t}, J=7.3 \mathrm{~Hz}, 2 \mathrm{H}), 2.51-2.43(\mathrm{~m}, 1 \mathrm{H}), 2.42-2.33(\mathrm{~m}, 1 \mathrm{H}), 2.14(\mathrm{~s}, 3 \mathrm{H}), 1.77(\mathrm{~s}, 3 \mathrm{H}), 1.35(\mathrm{t}, J=7.1 \mathrm{~Hz}, 3 \mathrm{H})$. 
${ }^{13} \mathrm{C}$ NMR $\left(125 \mathrm{MHz}, \mathrm{CDCl}_{3}\right) \delta 208.61,152.47,136.05,128.04,124.99,122.14,119.31,119.19,117.82,114.90$, 109.14, 72.25, 65.23, 44.14, 41.26, 39.87, 30.13, 24.70, 19.19, 14.21. HRMS (ESI): calcd. for $\mathrm{C}_{20} \mathrm{H}_{24} \mathrm{~N}_{2} \mathrm{O} 4\left([\mathrm{M}+\mathrm{H}]^{+}\right): 357.1800$, found 357.1804

\section{Preparation of 1x:}

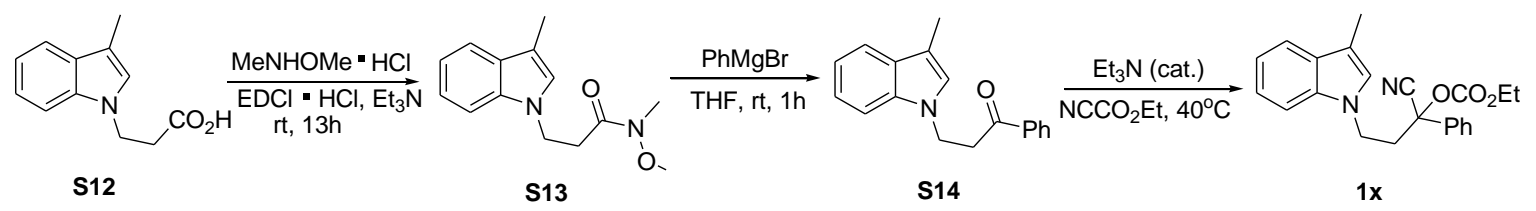

To a solution of $\mathbf{S 1 2}$ (1.0 equiv.), $\mathrm{Et}_{3} \mathrm{~N}$ (2.8 equiv.) and $\mathrm{N}, \mathrm{O}$-dimethylhydroxyamine hydrochloride (1.1 equiv.) in dichloromethane $(0.2 \mathrm{M})$ was added 1-ethyl-3-(3-dimethyllaminopropyl) hydrochloride (1.2 equiv.). ${ }^{4}$ The mixture was stirred for $13 \mathrm{~h}$ at $\mathrm{rt}$ before being quenched with water. The aqueous phase extracted with EtOAc. The combined organic phases were washed with $\mathrm{HCl}(1 \mathrm{M})$ and brine before being dried over $\mathrm{Na}_{2} \mathrm{SO}_{4}$ and concentrated in vacuo. The residue was purified by column chromatography (silica gel, EtOAc/Petroleum ether) to afford S13.

To a solution of $\mathbf{S 1 3}$ (1.0 equiv.3.0 mmo1) in dry THF $(0.5 \mathrm{M})$ was added $\mathrm{PhMgBr}$ (1.5 equiv.) at $0{ }^{\circ} \mathrm{C}$ under nitrogen atmosphere. The reaction mixture was stirred at $\mathrm{rt}$ for $1 \mathrm{~h}$. Upon completion, the reaction was quenched with saturated ammonium chloride solution at $0{ }^{\circ} \mathrm{C}$ and the mixture was extracted with DCM. The combined organic phases were washed with brine before being dried $\left(\mathrm{Na}_{2} \mathrm{SO}_{4}\right)$ and concentrated in vacuo. Purification by column chromatography (silica gel, EtOAc/Petroleum ether) to afford the ketone S14.

Compounds 1x were prepared from S14 according to the procedure for the synthesis of compound $\mathbf{1 a}$.<smiles>CCOC(=O)c1ccccc1CC(C)(C)C(=O)OCC</smiles>

1x

Colorless oil. ${ }^{1} \mathrm{H}$ NMR (500 MHz, $\left.\mathrm{CDCl}_{3}\right) \delta$ 7.56-7.50 (m, 3H), $7.46-7.38(\mathrm{~m}, 3 \mathrm{H}), 7.22-7.16(\mathrm{~m}, 2 \mathrm{H}), 7.13-$ $7.05(\mathrm{~m}, 1 \mathrm{H}), 6.79(\mathrm{~s}, 1 \mathrm{H}), 4.44-4.35(\mathrm{~m}, 1 \mathrm{H}), 4.31-4.08(\mathrm{~m}, 3 \mathrm{H}), 2.73-2.65(\mathrm{~m}, 1 \mathrm{H}), 2.53-2.45(\mathrm{~m}, 1 \mathrm{H}), 2.27(\mathrm{~s}$, $3 \mathrm{H}), 1.29(\mathrm{t}, J=7.1 \mathrm{~Hz}, 3 \mathrm{H}) .{ }^{13} \mathrm{C} \mathrm{NMR}\left(125 \mathrm{MHz}, \mathrm{CDCl}_{3}\right) \delta 152.19,136.15,135.99,129.91,129.34,129.10$, $125.12,124.69,121.92,119.33,119.07,116.79,111.36,108.85,77.51,65.49,42.72,41.52,14.19,9.64$. HRMS (ESI): calcd. for $\mathrm{C}_{22} \mathrm{H}_{22} \mathrm{~N}_{2} \mathrm{O}_{3}\left([\mathrm{M}+\mathrm{H}]^{+}\right)$: 363.1703 , found 363.1700 .

Preparation of 3a-3b, 5a-5b, 7a-7b:

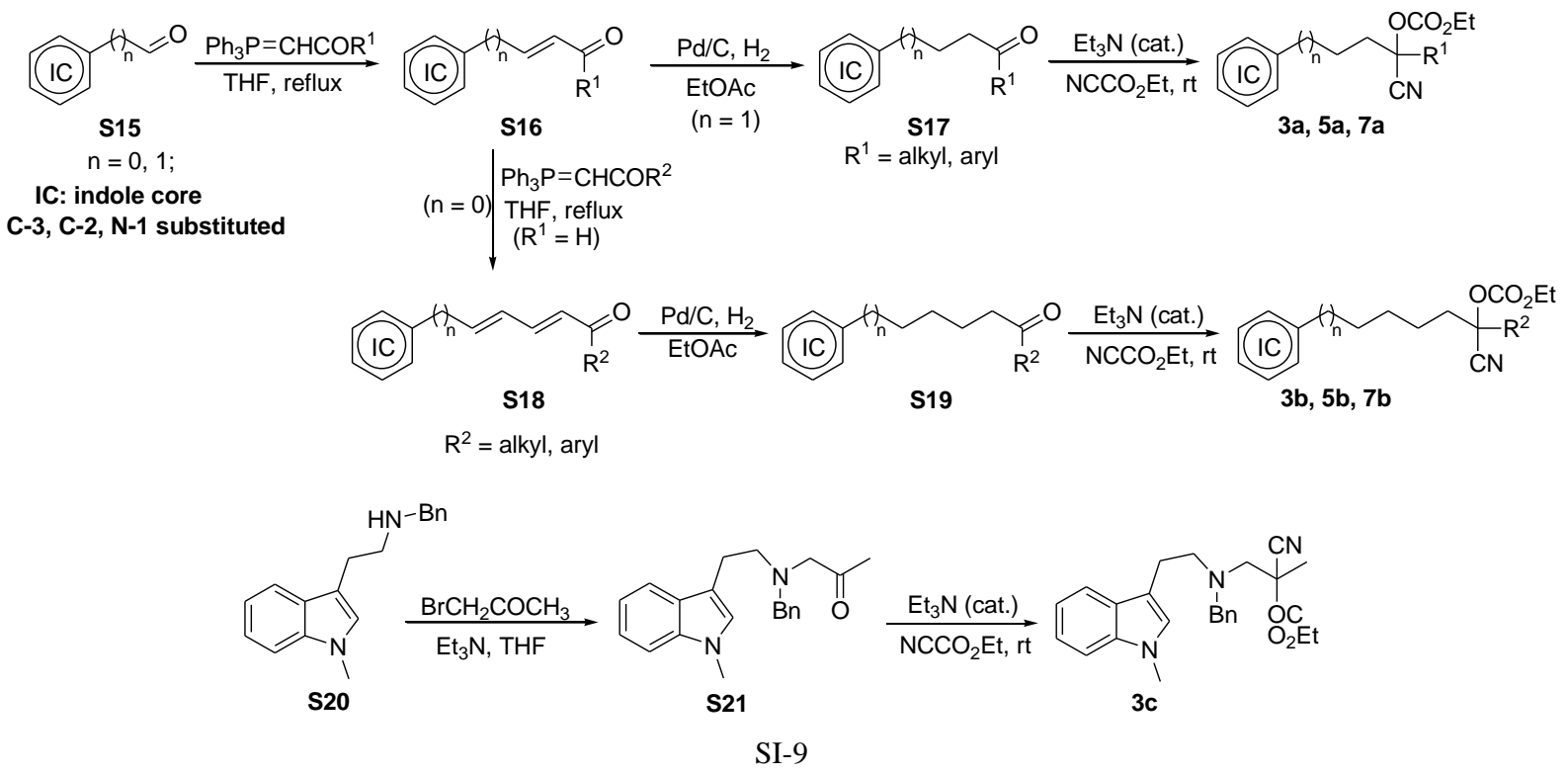


Corresponding $\mathbf{S 1 5}$ (1.0 equiv.) was dissolved in dry THF $(0.4 M)$ and $\mathrm{Ph}_{3} \mathrm{P}=\mathrm{CHCOR}^{1}$ (1.0 equiv.) was added. The mixture was heated to reflux and stirring was maintained until the reaction was completed. The solvent was evaporated in vacuo and the residue was purified by column chromatography (silica gel, EtOAc/Petroleum ether) to give S16. $^{7}$

S16 (1.0 equiv., $\mathrm{R}^{1}=\mathrm{Me}$ or $\mathrm{Ph}$ ) was dissolved in EtOAc $(0.2 \mathrm{M})$, palladium on carbon $(5 \% \mathrm{Pd})$ was added and the reaction was stirred for 2 hours under a $\mathrm{H}_{2}$ atmosphere. Then the reaction was diluted with $\mathrm{CH}_{2} \mathrm{Cl}_{2}$ and filtered through a short Celite ${ }^{\circledR}$ pad, and the filtrate was concentrated under reduced pressure, The residue was purified by column chromatography (silica gel, EtOAc/Petroleum ether) to give S17.

S18 was prepared for according to the procedure for the synthesis of compound S16 from S15, while S19 was prepared for according to the procedure for the synthesis of compound S17 from S16.

Compounds 3a-3b, 5a-5b and $\mathbf{7 a - 7 b}$ were prepared from S17 and S19 according to the procedure for the synthesis of compound 1a. Compound 3c was prepared from S21. 1-Bromopropan-2-one (1.5eq) was added to a solution of $\mathbf{S 2 0}$ (1eq) and $\mathrm{Et}_{3} \mathrm{~N}$ (2eq) in THF (0.4M). The reaction mixture was stirred for $6 \mathrm{~h}$, filtered, and concentrated in vacuo. The residue was purified by column chromatography (Silica gel, EtOAc/petroleum ether) to give $\mathbf{S 2 1}$.

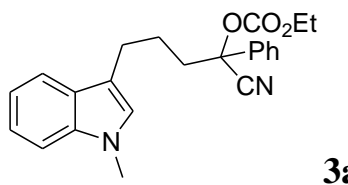

Colorless oil. ${ }^{1} \mathrm{H}$ NMR $\left(500 \mathrm{MHz}, \mathrm{CDCl}_{3}\right) \delta 7.38(\mathrm{~d}, J=6.4 \mathrm{~Hz}, 3 \mathrm{H}), 7.27(\mathrm{~d}, J=7.7 \mathrm{~Hz}, 3 \mathrm{H}), 7.15(\mathrm{~d}, J=8.0 \mathrm{~Hz}$, 1H), $7.12-7.06(\mathrm{~m}, 1 \mathrm{H}), 6.98-6.95(\mathrm{~m}, 1 \mathrm{H}), 6.64(\mathrm{~s}, 1 \mathrm{H}), 4.12-3.95(\mathrm{~m}, 2 \mathrm{H}), 3.56(\mathrm{~s}, 3 \mathrm{H}), 2.69-2.60(\mathrm{~m}, 2 \mathrm{H})$, 2.28-2.23 (m, 1H), 2.07-2.02 (m, 1H), 1.97-1.87 (m, 1H), 1.72-1.60 (m, 1H), $1.13(\mathrm{t}, J=7.1 \mathrm{~Hz}, 3 \mathrm{H}) .{ }^{13} \mathrm{C}$ NMR $\left(125 \mathrm{MHz}, \mathrm{CDCl}_{3}\right) \delta 152.29,137.05,136.94,129.33,128.95,127.75,126.22,124.81,121.55,118.84,118.71$, 117.32, 113.61, 109.18, 79.54, 64.99, 42.25, 32.53, 24.72, 24.35, 14.07. HRMS (ESI): calcd. for $\mathrm{C}_{23} \mathrm{H}_{24} \mathrm{~N}_{2} \mathrm{O}_{3}$ $\left([\mathrm{M}+\mathrm{H}]^{+}\right): 377.1860$, found 377.1859 .

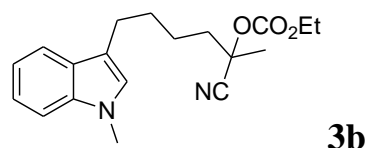

Yellow oil. ${ }^{1} \mathrm{H}$ NMR $\left(500 \mathrm{MHz}, \mathrm{CDCl}_{3}\right) \delta 7.56(\mathrm{~d}, J=7.9 \mathrm{~Hz}, 1 \mathrm{H}), 7.28(\mathrm{~d}, J=8.2 \mathrm{~Hz}, 1 \mathrm{H}), 7.25-7.17(\mathrm{~m}, 1 \mathrm{H})$, $7.09(\mathrm{t}, J=7.4 \mathrm{~Hz}, 1 \mathrm{H}), 6.82(\mathrm{~s}, 1 \mathrm{H}), 4.24(\mathrm{q}, J=7.1 \mathrm{~Hz}, 2 \mathrm{H}), 3.72(\mathrm{~s}, 3 \mathrm{H}), 2.77(\mathrm{t}, J=7.3 \mathrm{~Hz}, 2 \mathrm{H}), 2.10-2.04(\mathrm{~m}$, $1 \mathrm{H}), 1.97-1.91(\mathrm{~m}, 1 \mathrm{H}), 1.80-1.72(\mathrm{~m}, 2 \mathrm{H}), 1.75(\mathrm{~s}, 3 \mathrm{H}), 1.71-1.65(\mathrm{~m}, 1 \mathrm{H}), 1.64-1.55(\mathrm{~m}, 1 \mathrm{H}), 1.32(\mathrm{t}, J=7.1$ $\mathrm{Hz}, 3 \mathrm{H}) .{ }^{13} \mathrm{C} \mathrm{NMR}\left(125 \mathrm{MHz}, \mathrm{CDCl}_{3}\right) \delta 152.68,137.11,127.81,126.23,121.55,118.99,118.63,114.59,109.26$, $74.20,64.88,39.57,32.65,29.96,24.86,24.51,23.77,14.23$. HRMS (ESI): calcd. for $\mathrm{C}_{19} \mathrm{H}_{24} \mathrm{~N}_{2} \mathrm{O}_{3}\left([\mathrm{M}+\mathrm{H}]^{+}\right)$: 329.1860 , found 329.1867 .

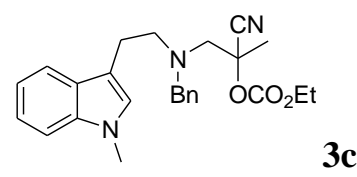

Colourless oil, ${ }^{1} \mathrm{H}$ NMR $\left(500 \mathrm{MHz}, \mathrm{CDCl}_{3}\right) \delta$ 7.43-7.36 (m, 3H), $7.32(\mathrm{t}, J=7.4 \mathrm{~Hz}, 2 \mathrm{H}), 7.28-7.22(\mathrm{~m}, 2 \mathrm{H}), 7.17$ (t, $J=7.6 \mathrm{~Hz}, 1 \mathrm{H}), 7.03(\mathrm{t}, J=7.4 \mathrm{~Hz}, 1 \mathrm{H}), 6.76(\mathrm{~s}, 1 \mathrm{H}), 4.22(\mathrm{q}, J=7.1 \mathrm{~Hz}, 2 \mathrm{H}), 3.99(\mathrm{~d}, J=13.8 \mathrm{~Hz}, 1 \mathrm{H}), 3.87$ 
$(\mathrm{d}, J=13.8 \mathrm{~Hz}, 1 \mathrm{H}), 3.68(\mathrm{~s}, 3 \mathrm{H}), 3.23(\mathrm{~d}, J=14.4 \mathrm{~Hz}, 1 \mathrm{H}), 3.09(\mathrm{~d}, J=14.4 \mathrm{~Hz}, 1 \mathrm{H}), 3.00-2.89(\mathrm{~m}, 4 \mathrm{H}), 1.71(\mathrm{~s}$, $3 \mathrm{H}), 1.29(\mathrm{t}, J=7.1 \mathrm{~Hz}, 3 \mathrm{H}) .{ }^{13} \mathrm{C}$ NMR $\left(125 \mathrm{MHz}, \mathrm{CDCl}_{3}\right) \delta 152.76,138.92,137.03,129.18,128.49,127.93$, $127.38,126.59,121.59,118.98,118.84,118.74,112.34,109.24,75.38,64.94,61.14,60.04,56.05,32.66,22.95$, 22.88, 14.26. HRMS (ESI): calcd. for $\mathrm{C}_{25} \mathrm{H}_{29} \mathrm{~N}_{3} \mathrm{O}_{3}\left([\mathrm{M}+\mathrm{H}]^{+}\right)$: 420.2282, found 420.2284.

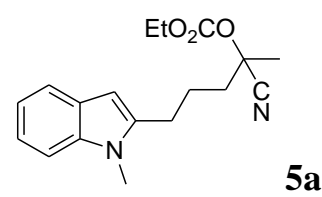

Colourless oil. ${ }^{1} \mathrm{H}$ NMR $\left(500 \mathrm{MHz}, \mathrm{CDCl}_{3}\right) \delta 7.54(\mathrm{~d}, J=7.6 \mathrm{~Hz}, 1 \mathrm{H}), 7.26(\mathrm{~d}, J=8.1 \mathrm{~Hz}, 1 \mathrm{H}), 7.16(\mathrm{t}, J=7.4 \mathrm{~Hz}$, 1H), $7.07(\mathrm{t}, J=7.1 \mathrm{~Hz}, 1 \mathrm{H}), 6.27(\mathrm{~s}, 1 \mathrm{H}), 4.24(\mathrm{q}, J=7.0 \mathrm{~Hz}, 2 \mathrm{H}), 3.64(\mathrm{~s}, 3 \mathrm{H}), 2.88-2.75(\mathrm{~m}, 2 \mathrm{H}), 2.18-2.08$ $(\mathrm{m}, 1 \mathrm{H}), 2.07-1.89(\mathrm{~m}, 3 \mathrm{H}), 1.78(\mathrm{~s}, 3 \mathrm{H}), 1.32(\mathrm{t}, J=7.0 \mathrm{~Hz}, 3 \mathrm{H}) .{ }^{13} \mathrm{C} \mathrm{NMR}\left(125 \mathrm{MHz}, \mathrm{CDCl}_{3}\right) \delta 152.64,139.43$, 137.51, 127.80, 120.90, 119.93, 119.45, 118.42, 108.92, 99.31, 73.92, 64.94, 39.28, 29.54, 26.17, 24.56, 22.76, 14.20. HRMS (ESI): calcd. for $\mathrm{C}_{18} \mathrm{H}_{22} \mathrm{~N}_{2} \mathrm{O}_{3}\left([\mathrm{M}+\mathrm{H}]^{+}\right)$: 315.1703 , found 315.1708 .

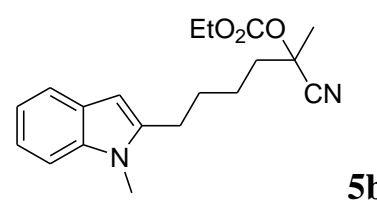

Colorless oil. ${ }^{1} \mathrm{H}$ NMR (500 MHz, $\left.\mathrm{CDCl}_{3}\right) \delta 7.53(\mathrm{~d}, J=7.8 \mathrm{~Hz}, 1 \mathrm{H}), 7.27(\mathrm{~d}, J=8.1 \mathrm{~Hz}, 1 \mathrm{H}), 7.19-7.13(\mathrm{~m}, 1 \mathrm{H})$, $7.09-7.05(\mathrm{~m}, 1 \mathrm{H}), 6.25(\mathrm{~s}, 1 \mathrm{H}), 4.25(\mathrm{q}, J=7.1 \mathrm{~Hz}, 2 \mathrm{H}), 3.67(\mathrm{~s}, 3 \mathrm{H}), 2.80-2.76(\mathrm{~m}, 2 \mathrm{H}), 2.15-2.06(\mathrm{~m}, 1 \mathrm{H})$, 2.02-1.95 (m, 1H), $1.79(\mathrm{~s}, 3 \mathrm{H}), 1.84-1.62(\mathrm{~m}, 4 \mathrm{H}), 1.33(\mathrm{t}, J=7.1 \mathrm{~Hz}, 3 \mathrm{H}) .{ }^{13} \mathrm{C}$ NMR $\left(125 \mathrm{MHz}, \mathrm{CDCl}_{3}\right) \delta$ 152.68, 140.44, 137.42, 127.87, 120.77, 119.90, 119.39, 118.54, 108.87, 98.91, 74.05, 64.98, 39.60, 29.55, 28.21, 26.67, 24.58, 23.79, 14.24. HRMS (ESI): calcd. for $\mathrm{C}_{19} \mathrm{H}_{24} \mathrm{~N}_{2} \mathrm{O}_{3}\left([\mathrm{M}+\mathrm{H}]^{+}\right)$: 329.1860, found 329.1867.

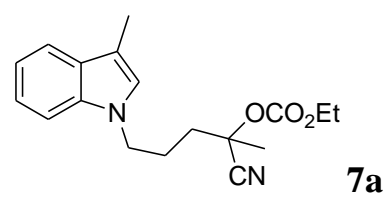

Yellow oil. ${ }^{1} \mathrm{H}$ NMR $\left(500 \mathrm{MHz}, \mathrm{CDCl}_{3}\right) \delta 7.57(\mathrm{~d}, J=7.7 \mathrm{~Hz}, 1 \mathrm{H}), 7.27(\mathrm{~d}, J=8.1 \mathrm{~Hz}, 1 \mathrm{H}), 7.20(\mathrm{t}, J=7.5 \mathrm{~Hz}$, $1 \mathrm{H}), 7.11(\mathrm{t}, J=7.3 \mathrm{~Hz}, 1 \mathrm{H}), 6.85(\mathrm{~s}, 1 \mathrm{H}), 4.23(\mathrm{q}, J=7.1 \mathrm{~Hz}, 2 \mathrm{H}), 4.18-4.07(\mathrm{~m}, 2 \mathrm{H}), 2.32(\mathrm{~s}, 3 \mathrm{H}), 2.20-2.02$ $(\mathrm{m}, 2 \mathrm{H}), 2.02-1.95(\mathrm{~m}, 1 \mathrm{H}), 1.91-1.82(\mathrm{~m}, 1 \mathrm{H}), 1.71(\mathrm{~s}, 3 \mathrm{H}), 1.31(\mathrm{t}, J=7.1 \mathrm{~Hz}, 3 \mathrm{H}) .{ }^{13} \mathrm{C}$ NMR $(125 \mathrm{MHz}$, $\left.\mathrm{CDCl}_{3}\right) \delta 152.56,136.30,128.90,125.15,121.73,119.27,118.84,118.26,110.95,109.03,73.68,65.06,45.16$, 37.23, 24.98, 24.51, 14.22, 9.72. HRMS (ESI): calcd. for $\mathrm{C}_{18} \mathrm{H}_{22} \mathrm{~N}_{2} \mathrm{O}_{3}\left([\mathrm{M}+\mathrm{H}]^{+}\right)$: 315.1703 , found 315.1704 .

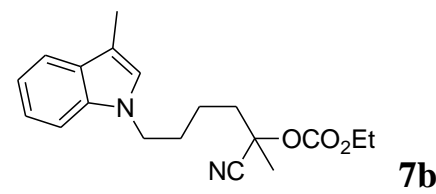

Yellow oil. ${ }^{1} \mathrm{H}$ NMR $\left(500 \mathrm{MHz}, \mathrm{CDCl}_{3}\right) \delta 7.56(\mathrm{~d}, J=7.9 \mathrm{~Hz}, 1 \mathrm{H}), 7.26(\mathrm{~d}, J=8.2 \mathrm{~Hz}, 1 \mathrm{H}), 7.21-7.17(\mathrm{~m}, 1 \mathrm{H})$, 7.12-7.16 (m, 1H), $6.84(\mathrm{~s}, 1 \mathrm{H}), 4.23(\mathrm{q}, J=7.1 \mathrm{~Hz}, 2 \mathrm{H}), 4.11-4.02(\mathrm{~m}, 2 \mathrm{H}), 2.31(\mathrm{~s}, 3 \mathrm{H}), 2.04-1.96(\mathrm{~m}, 1 \mathrm{H}), 1.93-$ $1.81(\mathrm{~m}, 3 \mathrm{H}), 1.71(\mathrm{~s}, 3 \mathrm{H}), 1.68-1.57(\mathrm{~m}, 1 \mathrm{H}), 1.57-1.48(\mathrm{~m}, 1 \mathrm{H}), 1.32(\mathrm{t}, J=7.1 \mathrm{~Hz}, 3 \mathrm{H}) .{ }^{13} \mathrm{C} \mathrm{NMR}(125 \mathrm{MHz}$, $\left.\mathrm{CDCl}_{3}\right) \delta 152.56,136.30,128.90,125.15,121.73,119.27,118.67,118.84,118.26,110.95,109.03,73.68,65.06$, 45.16, 37.23, 24.98, 24.51, 14.22, 9.72. HRMS (ESI): calcd. for $\mathrm{C}_{19} \mathrm{H}_{24} \mathrm{~N}_{2} \mathrm{O}_{3}\left([\mathrm{M}+\mathrm{H}]^{+}\right)$: 329.1860, found 329.1859. 


\section{Preparation of 9a-9j:}

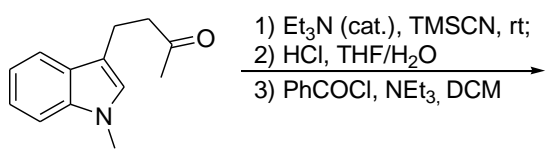

S2

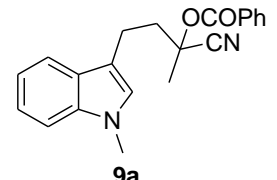

9a

To a mixture of $\mathbf{S 2}$ (1.0 equiv.) and $\mathrm{Et}_{3} \mathrm{~N}$ (10 mol\%), TMSCN (1.1 equiv.) was slowly added at $\mathrm{rt}^{2}{ }^{2}$ Upon completion, the reaction was concentrated in vacuum. The residue was treated with $\mathrm{THF}-\mathrm{HCl}$ ( $4 \mathrm{~N}$, aqueous solution) $(1 / 1)$, and the mixture was stirred at $\mathrm{rt}$. Upon completion, the aqueous phase extracted with EtOAc. The combined organic phases were washed with brine before being dried over $\mathrm{Na}_{2} \mathrm{SO}_{4}$ and concentrated in vacuo. The crude product was dissolved in $\mathrm{DCM}(M=0.2)$. To this solution, $\mathrm{PhCOCl}\left(1.2\right.$ equiv.) and $\mathrm{Et}_{3} \mathrm{~N}$ (2.0 equiv) were added at $0{ }^{\circ} \mathrm{C}$. Then, the reaction was allowed to warm to rt. Upon completion, the reaction was quenched with saturated ammonium chloride solution at $0{ }^{\circ} \mathrm{C}$ and the mixture was extracted with DCM. The combined organic phases were washed with brine before being dried $\left(\mathrm{Na}_{2} \mathrm{SO}_{4}\right)$ and concentrated in vacuo. Purification by column chromatography (silica gel, EtOAc/Petroleum ether) to afford compound $9 a$.

Compounds $9 \mathbf{b}-\mathbf{9 j}$ were prepared according to the procedure for the synthesis of compound $9 \mathbf{a}$.

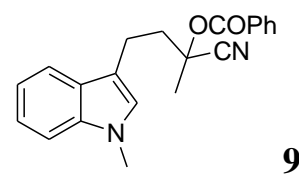

\section{9a}

Colourless oil. ${ }^{1} \mathrm{H}$ NMR $\left(500 \mathrm{MHz}, \mathrm{CDCl}_{3}\right) \delta 7.95(\mathrm{~d}, J=7.9 \mathrm{~Hz}, 2 \mathrm{H}), 7.63-7.58(\mathrm{~m}, 2 \mathrm{H}), 7.44(\mathrm{t}, J=7.7 \mathrm{~Hz}, 2 \mathrm{H})$, $7.31-7.23(\mathrm{~m}, 2 \mathrm{H}), 7.14(\mathrm{t}, J=7.4 \mathrm{~Hz}, 1 \mathrm{H}), 6.91(\mathrm{~s}, 1 \mathrm{H}), 3.72(\mathrm{~s}, 3 \mathrm{H}), 3.18-3.04(\mathrm{~m}, 2 \mathrm{H}), 3.11-3.04(\mathrm{~m}, 1 \mathrm{H})$, 2.61-2.55 (m, 1H), $2.47-2.41(\mathrm{~m}, 1 \mathrm{H}), 1.96(\mathrm{~s}, 3 \mathrm{H}) .{ }^{13} \mathrm{C} \mathrm{NMR}\left(125 \mathrm{MHz}, \mathrm{CDCl}_{3}\right) \delta 164.57,137.20,133.81$, 129.85, 129.18, 128.67, 127.49, 126.41, 121.90, 119.04, 118.84, 118.74, 112.66, 109.50, 72.37, 40.70, 32.70, 24.88, 20.09. HRMS (ESI): calcd. for $\mathrm{C}_{21} \mathrm{H}_{20} \mathrm{~N}_{2} \mathrm{O}_{2}\left([\mathrm{M}+\mathrm{H}]^{+}\right)$: 333.1598 , found 333.1600 .

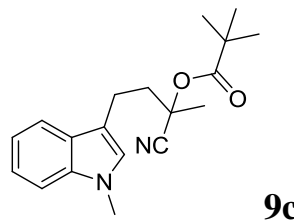

White solid, mp: 84.7-85.9 ${ }^{\circ} \mathrm{C} .{ }^{1} \mathrm{H}$ NMR $\left(500 \mathrm{MHz}, \mathrm{CDCl}_{3}\right) \delta 7.58(\mathrm{~d}, J=7.9 \mathrm{~Hz}, 1 \mathrm{H}), 7.31(\mathrm{~d}, J=8.2 \mathrm{~Hz}, 1 \mathrm{H})$, $7.28-7.21(\mathrm{~m}, 1 \mathrm{H}), 7.13(\mathrm{t}, J=7.8 \mathrm{~Hz}, 1 \mathrm{H}), 6.89(\mathrm{~s}, 1 \mathrm{H}), 3.75(\mathrm{~s}, 3 \mathrm{H}), 3.10-2.93(\mathrm{~m}, 2 \mathrm{H}), 3.06-2.94(\mathrm{~m}, 1 \mathrm{H})$, $2.31-2.25(\mathrm{~m}, 1 \mathrm{H}), 1.80(\mathrm{~s}, 3 \mathrm{H}), 1.25(\mathrm{~s}, 9 \mathrm{H}) .{ }^{13} \mathrm{C} \mathrm{NMR}\left(125 \mathrm{MHz}, \mathrm{CDCl}_{3}\right) \delta 176.50,137.19,127.49,126.35$, $121.91,119.04,118.93,118.68,112.71,109.49,71.42,40.87,39.30,32.74,27.06,24.56,19.86$. HRMS (ESI): calcd. for $\mathrm{C}_{19} \mathrm{H}_{24} \mathrm{~N}_{2} \mathrm{O}_{2}\left([\mathrm{M}+\mathrm{H}]^{+}\right): 313.1911$, found 313.1915.

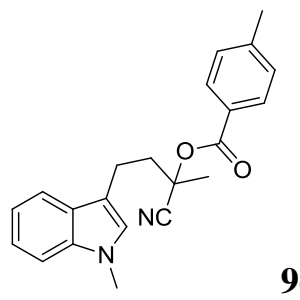


Yellow solid, mp: 205.6-206.6 ${ }^{\circ} \mathrm{C} .{ }^{1} \mathrm{H}$ NMR $\left(500 \mathrm{MHz}, \mathrm{CDCl}_{3}\right) \delta 7.85(\mathrm{~d}, J=8.0 \mathrm{~Hz}, 2 \mathrm{H}), 7.62(\mathrm{~d}, J=7.5 \mathrm{~Hz}, 1 \mathrm{H})$, $7.29(\mathrm{~d}, J=8.1 \mathrm{~Hz}, 1 \mathrm{H}), 7.26-7.23(\mathrm{~m}, 3 \mathrm{H}), 7.13(\mathrm{t}, J=7.4 \mathrm{~Hz}, 1 \mathrm{H}), 6.90(\mathrm{~s}, 1 \mathrm{H}), 3.72(\mathrm{~s}, 3 \mathrm{H}), 3.17-3.03(\mathrm{~m}$, $2 \mathrm{H}), 2.61-2.50(\mathrm{~m}, 1 \mathrm{H}), 2.48-2.36(\mathrm{~m}, 4 \mathrm{H}), 1.94(\mathrm{~s}, 3 \mathrm{H}) .{ }^{13} \mathrm{C} \mathrm{NMR}\left(125 \mathrm{MHz}, \mathrm{CDCl}_{3}\right) \delta 164.66,144.70,137.23$, $129.92,129.39,127.54,126.49,126.40,121.91,119.06,118.96,118.77,112.77,109.49,72.18,40.79,32.72,24.92$, 21.87, 20.08. HRMS (ESI): calcd. for $\mathrm{C}_{22} \mathrm{H}_{22} \mathrm{~N}_{2} \mathrm{O}_{2}\left([\mathrm{M}+\mathrm{H}]^{+}\right)$: 347.1754 found 347.1753.

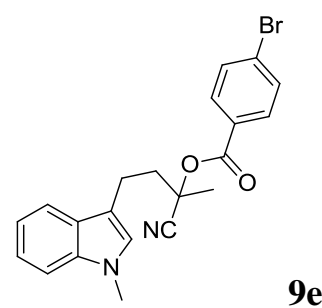

Yellow solid, mp: $140.9-150.6{ }^{\circ} \mathrm{C} .{ }^{1} \mathrm{H}$ NMR $\left(500 \mathrm{MHz}, \mathrm{CDCl}_{3}\right) \delta 7.75(\mathrm{~d}, J=8.4 \mathrm{~Hz}, 2 \mathrm{H}), 7.61(\mathrm{~d}, J=7.8 \mathrm{~Hz}, 1 \mathrm{H})$, $7.56(\mathrm{~d}, J=8.5 \mathrm{~Hz}, 2 \mathrm{H}), 7.32-7.21(\mathrm{~m}, 2 \mathrm{H}), 7.14(\mathrm{t}, J=7.1 \mathrm{~Hz}, 1 \mathrm{H}), 6.89(\mathrm{~s}, 1 \mathrm{H}), 3.71(\mathrm{~s}, 3 \mathrm{H}), 3.20-3.00(\mathrm{~m}$, $2 \mathrm{H}), 2.63-2.52(\mathrm{~m}, 1 \mathrm{H}), 2.48-2.39(\mathrm{~m}, 1 \mathrm{H}), 1.95(\mathrm{~s}, 3 \mathrm{H}) .{ }^{13} \mathrm{C} \mathrm{NMR}\left(125 \mathrm{MHz}, \mathrm{CDCl}_{3}\right) \delta 163.88,137.21,132.03$, 131.31, 129.07, 128.06, 127.47, 126.44, 121.97, 119.11, 118.73, 118.64, 112.55, 109.53, 72.77, 40.52, 32.73, 24.89, 20.17. HRMS (ESI): calcd. for $\mathrm{C}_{21} \mathrm{H}_{19} \mathrm{BrN}_{2} \mathrm{O}_{2}\left([\mathrm{M}+\mathrm{H}]^{+}\right)$: 411.0703, found 411.0700.

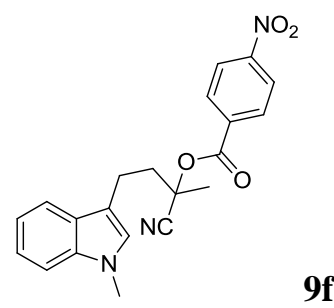

Colourless oil. ${ }^{1} \mathrm{H}$ NMR $\left(500 \mathrm{MHz}, \mathrm{CDCl}_{3}\right) \delta 8.23(\mathrm{~d}, J=8.4 \mathrm{~Hz}, 2 \mathrm{H}), 7.99(\mathrm{~d}, J=8.4 \mathrm{~Hz}, 2 \mathrm{H}), 7.61(\mathrm{~d}, J=7.7 \mathrm{~Hz}$, $1 \mathrm{H}), 7.28-7.23(\mathrm{~m}, 2 \mathrm{H}), 7.14(\mathrm{t}, J=6.8 \mathrm{~Hz}, 1 \mathrm{H}), 6.90(\mathrm{~s}, 1 \mathrm{H}), 3.69(\mathrm{~s}, 3 \mathrm{H}), 3.20-3.03(\mathrm{~m}, 2 \mathrm{H}), 2.67-2.57(\mathrm{~m}$, $1 \mathrm{H}), 2.53-2.43(\mathrm{~m}, 1 \mathrm{H}), 1.98(\mathrm{~s}, 3 \mathrm{H}) .{ }^{13} \mathrm{C} \mathrm{NMR}\left(125 \mathrm{MHz}, \mathrm{CDCl}_{3}\right) \delta 162.70,150.91,137.20,134.40,130.92$, 127.42, 126.50, 123.71, 122.03, 119.17, 118.69, 118.27, 112.36, 109.55, 73.60, 40.26, 32.72, 24.85, 20.25. HRMS (ESI): calcd. for $\mathrm{C}_{21} \mathrm{H}_{20} \mathrm{~N}_{3} \mathrm{O}_{4}\left([\mathrm{M}+\mathrm{H}]^{+}\right): 378.1448$ found 378.1446 .

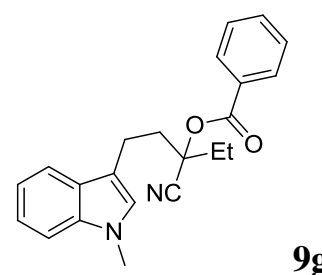

Colourless oil. ${ }^{1} \mathrm{H}$ NMR $\left(500 \mathrm{MHz}, \mathrm{CDCl}_{3}\right) \delta 7.96(\mathrm{~d}, J=7.6 \mathrm{~Hz}, 2 \mathrm{H}), 7.59(\mathrm{t}, J=7.3 \mathrm{~Hz}, 2 \mathrm{H}), 7.44(\mathrm{t}, J=7.5 \mathrm{~Hz}$, $2 \mathrm{H}), 7.30-7.19(\mathrm{~m}, 2 \mathrm{H}), 7.12(\mathrm{t}, J=7.0 \mathrm{~Hz}, 1 \mathrm{H}), 6.88(\mathrm{~s}, 1 \mathrm{H}), 3.69(\mathrm{~s}, 3 \mathrm{H}), 3.16-2.96(\mathrm{~m}, 2 \mathrm{H}), 2.64-2.54(\mathrm{~m}$, $1 \mathrm{H}), 2.53-2.42(\mathrm{~m}, 1 \mathrm{H}), 2.39-2.32(\mathrm{~m}, 1 \mathrm{H}), 2.28-2.21(\mathrm{~m} \mathrm{1H}), 1.17(\mathrm{t}, J=7.3 \mathrm{~Hz}, 3 \mathrm{H}) .{ }^{13} \mathrm{C} \mathrm{NMR}(125 \mathrm{MHz}$, $\left.\mathrm{CDCl}_{3}\right) \delta 164.51,137.16,133.78,129.82,129.22,128.68,127.49,126.39,121.86,119.03,118.73,118.31,112.73$, 109.46, 76.79, 37.19, 32.68, 30.26, 19.87, 8.36. HRMS (ESI): calcd. for $\mathrm{C}_{22} \mathrm{H}_{22} \mathrm{~N}_{2} \mathrm{O}_{2}\left([\mathrm{M}+\mathrm{H}]^{+}\right): 347.1754$, found 347.1757. 


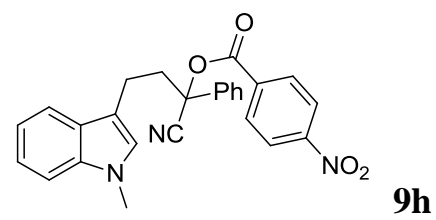

Yellow solid, mp: $145.4-146.3{ }^{\circ} \mathrm{C} .{ }^{1} \mathrm{H}$ NMR $\left(500 \mathrm{MHz}, \mathrm{CDCl}_{3}\right) \delta 8.20(\mathrm{~d}, J=8.7 \mathrm{~Hz}, 2 \mathrm{H}), 8.00(\mathrm{~d}, J=8.7 \mathrm{~Hz}, 2 \mathrm{H})$, $7.61(\mathrm{~d}, J=7.1 \mathrm{~Hz}, 2 \mathrm{H}), 7.51(\mathrm{~d}, J=7.8 \mathrm{~Hz}, 1 \mathrm{H}), 7.45-7.39(\mathrm{~m}, 3 \mathrm{H}), 7.29-7.19(\mathrm{~m}, 2 \mathrm{H}), 7.11(\mathrm{t}, J=7.2 \mathrm{~Hz}, 1 \mathrm{H})$, $6.86(\mathrm{~s}, 1 \mathrm{H}), 3.67(\mathrm{~s}, 3 \mathrm{H}), 3.24-3.10(\mathrm{~m}, 1 \mathrm{H}), 2.98-2.80(\mathrm{~m}, 2 \mathrm{H}), 2.68-2.56(\mathrm{~m}, 1 \mathrm{H}) .{ }^{13} \mathrm{C} \mathrm{NMR}(125 \mathrm{MHz}$, $\left.\mathrm{CDCl}_{3}\right) \delta 162.28,150.95,137.16,136.62,134.09,130.99,129.67,129.26,127.38,126.49,124.86,123.76,121.96$, 119.11, 118.62, 116.97, 112.27, 109.51, 78.55, 43.07, 32.68, 20.49. HRMS (ESI): calcd. for $\mathrm{C}_{26} \mathrm{H}_{21} \mathrm{~N}_{3} \mathrm{O}_{4}\left([\mathrm{M}+\mathrm{H}]^{+}\right)$: 440.1605 , found 440.1600 .

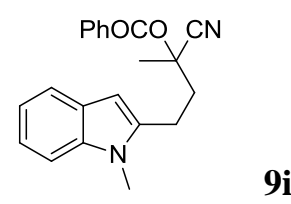

Colourless oil. ${ }^{1} \mathrm{H}$ NMR $\left(500 \mathrm{MHz}, \mathrm{CDCl}_{3}\right) \delta 7.97(\mathrm{~d}, J=7.3 \mathrm{~Hz}, 2 \mathrm{H}), 7.61(\mathrm{t}, J=7.4 \mathrm{~Hz}, 1 \mathrm{H}), 7.55(\mathrm{~d}, J=7.8 \mathrm{~Hz}$, $1 \mathrm{H}), 7.44(\mathrm{t}, J=7.8 \mathrm{~Hz}, 2 \mathrm{H}), 7.28(\mathrm{~d}, J=8.1 \mathrm{~Hz}, 1 \mathrm{H}), 7.19(\mathrm{t}, J=7.4 \mathrm{~Hz}, 1 \mathrm{H}), 7.09(\mathrm{t}, J=7.3 \mathrm{~Hz}, 1 \mathrm{H}), 6.33(\mathrm{~s}$, $1 \mathrm{H}), 3.72(\mathrm{~s}, 3 \mathrm{H}), 3.22-3.02(\mathrm{~m}, 2 \mathrm{H}), 2.66-2.60(\mathrm{~m}, 1 \mathrm{H}), 2.50-2.44(\mathrm{~m}, 1 \mathrm{H}), 1.99(\mathrm{~s}, 3 \mathrm{H}) .{ }^{13} \mathrm{C} \mathrm{NMR}(125 \mathrm{MHz}$, $\left.\mathrm{CDCl}_{3}\right) \delta 164.51,138.33,137.60,134.00,129.88,128.96,128.76,127.78,121.26,120.09,119.67,118.54,109.03$, 99.27, 72.03, 39.15, 29.65, 24.96, 21.85. HRMS (ESI): calcd. for $\mathrm{C}_{21} \mathrm{H}_{20} \mathrm{~N}_{2} \mathrm{O}_{2}\left([\mathrm{M}+\mathrm{H}]^{+}\right)$: 333.1598, found 333.1593.

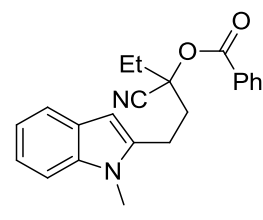

\section{9j}

Colourless oil. ${ }^{1} \mathrm{H}$ NMR $\left(500 \mathrm{MHz}, \mathrm{CDCl}_{3}\right) \delta 7.98(\mathrm{~d}, J=7.6 \mathrm{~Hz}, 2 \mathrm{H}), 7.60(\mathrm{t}, J=7.2 \mathrm{~Hz}, 1 \mathrm{H}), 7.53(\mathrm{~d}, J=7.7 \mathrm{~Hz}$, $1 \mathrm{H}), 7.44(\mathrm{t}, J=7.6 \mathrm{~Hz}, 2 \mathrm{H}), 7.25(\mathrm{t}, J=6.6 \mathrm{~Hz}, 1 \mathrm{H}), 7.17(\mathrm{t}, J=7.5 \mathrm{~Hz}, 1 \mathrm{H}), 7.07(\mathrm{t}, J=7.3 \mathrm{~Hz}, 1 \mathrm{H}), 6.30(\mathrm{~s}, 1 \mathrm{H})$, $3.68(\mathrm{~s}, 3 \mathrm{H}), 3.14-2.96(\mathrm{~m}, 2 \mathrm{H}), 2.72-2.57(\mathrm{~m}, 1 \mathrm{H}), 2.55-2.45(\mathrm{~m}, 1 \mathrm{H}), 2.40-2.33(\mathrm{~m}, 1 \mathrm{H}), 2.27(\mathrm{~m}, 1 \mathrm{H}), 2.30$ $-2.23(\mathrm{t}, J=7.3 \mathrm{~Hz}, 3 \mathrm{H}) .{ }^{13} \mathrm{C} \mathrm{NMR}\left(125 \mathrm{MHz}, \mathrm{CDCl}_{3}\right) \delta 164.49,138.40,137.59,129.86,128.98,128.78,127.75$, $121.23,120.07,119.65,118.01,109.01,99.28,76.43,35.75,30.39,29.65,21.73,8.35$. HRMS (ESI): calcd. for $\mathrm{C}_{22} \mathrm{H}_{22} \mathrm{~N}_{2} \mathrm{O}_{2}\left([\mathrm{M}+\mathrm{H}]^{+}\right): 347.1754$ found 347.1758 .

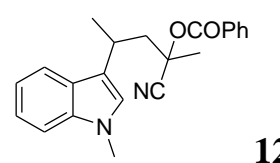

Colourless oil, $\mathrm{dr}=1 / 1\left(\mathbf{1 2 a} / \mathbf{1 2 b}=1 / 1\right.$, separated by column chromatography). The diastereoisomer 12a: ${ }^{1} \mathrm{H} \mathrm{NMR}$ $\left(500 \mathrm{MHz}, \mathrm{CDCl}_{3}\right) \delta 7.70(\mathrm{~d}, J=7.9 \mathrm{~Hz}, 1 \mathrm{H}), 7.47(\mathrm{t}, J=7.4 \mathrm{~Hz}, 1 \mathrm{H}), 7.40(\mathrm{~d}, J=7.4 \mathrm{~Hz}, 2 \mathrm{H}), 7.23(\mathrm{~m}, 4 \mathrm{H})$, 7.25-7.21 (m, 1H), 7.13-7.10 (s, 1H), $3.64-3.46(\mathrm{~m}, 1 \mathrm{H}), 3.55(\mathrm{~s}, 3 \mathrm{H}), 2.83(\mathrm{dd}, J=14.6,8.6 \mathrm{~Hz}, 1 \mathrm{H}), 2.31(\mathrm{dd}, J$ $=14.6,5.0 \mathrm{~Hz}, 1 \mathrm{H}), 1.87(\mathrm{~s}, 3 \mathrm{H}), 1.54(\mathrm{~d}, J=7.0 \mathrm{~Hz}, 3 \mathrm{H}) .{ }^{13} \mathrm{C} \mathrm{NMR}\left(125 \mathrm{MHz}, \mathrm{CDCl}_{3}\right) \delta 164.40,137.17,133.45$, 129.57, 129.02, 128.37, 126.38, 125.39, 121.67, 119.17, 119.05, 119.02, 118.86, 109.49, 72.43, 45.97, 32.45, 27.56, 25.10, 23.37. HRMS (ESI): calcd. for $\mathrm{C}_{22} \mathrm{H}_{22} \mathrm{~N}_{2} \mathrm{O}_{2}\left([\mathrm{M}+\mathrm{H}]^{+}\right): 347.1754$ found 347.1751. The diastereoisomer 12b: ${ }^{1} \mathrm{H}$ NMR $\left(500 \mathrm{MHz}, \mathrm{CDCl}_{3}\right) \delta 7.72(\mathrm{~d}, J=8.1 \mathrm{~Hz}, 2 \mathrm{H}), 7.66(\mathrm{~d}, J=7.8 \mathrm{~Hz}, 1 \mathrm{H}), 7.52(\mathrm{t}, J=7.4 \mathrm{~Hz}, 1 \mathrm{H}), 7.33(\mathrm{t}, J$ $=7.7 \mathrm{~Hz}, 2 \mathrm{H}), 7.21-7.14(\mathrm{~m}, 2 \mathrm{H}), 7.11-7.08(\mathrm{~m}, 1 \mathrm{H}), 6.83(\mathrm{~s}, 1 \mathrm{H}), 3.56(\mathrm{~s}, 3 \mathrm{H}), 3.56-3.49(\mathrm{~m}, 1 \mathrm{H}), 2.82-2.67(\mathrm{~m}$, 
1H), 2.46-2.42 (m, 1H), $1.80(\mathrm{~s}, 3 \mathrm{H}), 1.53(\mathrm{~d}, J=6.9 \mathrm{~Hz}, 3 \mathrm{H}) .{ }^{13} \mathrm{C} \mathrm{NMR}\left(125 \mathrm{MHz}, \mathrm{CDCl}_{3}\right) \delta$ 164.43, 137.18, 133.49 , 129.61, 129.04, 128.40, 126.40, 125.41, 121.71, 119.21, 119.08, 119.05, 118.89, 109.52, 72.46, 45.99, 32.50, 27.60, 25.14, 23.40. HRMS (ESI): calcd. for $\mathrm{C}_{22} \mathrm{H}_{22} \mathrm{~N}_{2} \mathrm{O}_{2}\left([\mathrm{M}+\mathrm{H}]^{+}\right): 347.1754$ found 347.1755. 


\section{Reaction Condition Screening}

Table S1. Screening of reaction conditions ${ }^{a}$

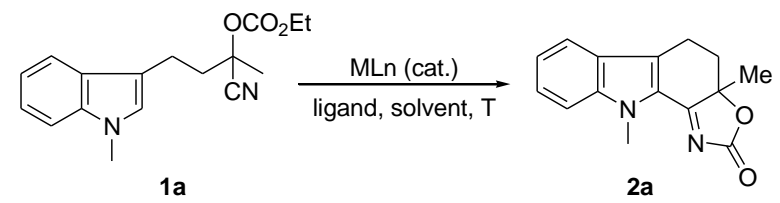

\begin{tabular}{|c|c|c|c|c|c|c|c|c|}
\hline Entry & Cat. & Ligand & additive & solvent & $\mathrm{T}\left({ }^{\circ} \mathrm{C}\right)$ & $\mathrm{t}(\mathrm{h})$ & Yield $(\%)^{b}$ & Con. $(\%)^{c}$ \\
\hline 1 & $\mathrm{Pd}(\mathrm{OAc})_{2}$ & bpy & D-CSA(1.5eq) & NMA & 120 & 24 & 21 & 99 \\
\hline 2 & $\mathrm{Pd}(\mathrm{OAc})_{2}$ & bpy & - & NMA & 120 & 24 & 75 & 85 \\
\hline 3 & $\mathrm{Pd}(\mathrm{OAc})_{2}$ & bpy & - & 1,4-dioxane & 120 & 24 & 34 & 48 \\
\hline 4 & $\mathrm{Pd}(\mathrm{OAc})_{2}$ & bpy & - & THF & 120 & 24 & 9 & 15 \\
\hline 5 & $\mathrm{Pd}(\mathrm{OAc})_{2}$ & bpy & - & DMF & 120 & 24 & 14 & 20 \\
\hline 6 & $\mathrm{Pd}(\mathrm{OAc})_{2}$ & bpy & - & DMSO & 120 & 24 & 51 & 73 \\
\hline 7 & $\mathrm{Pd}(\mathrm{OAc})_{2}$ & bpy & - & NMP & 120 & 24 & 26 & 40 \\
\hline 8 & $\mathrm{Pd}(\mathrm{OAc})_{2}$ & bpy & - & DMA & 120 & 24 & 25 & 28 \\
\hline 9 & $\operatorname{Pd}(\mathrm{TFA})_{2}$ & bpy & - & NMA & 120 & 24 & 38 & 83 \\
\hline 10 & $\mathrm{Pd}\left(\mathrm{Ph}_{3} \mathrm{P}\right)_{2} \mathrm{Cl}_{2}$ & bpy & - & NMA & 120 & 24 & 0 & 0 \\
\hline 11 & $\mathrm{Pd}(\mathrm{OAc})_{2}$ & phen & - & NMA & 120 & 24 & 78 & 100 \\
\hline 12 & $\mathrm{Pd}(\mathrm{OAc})_{2}$ & bpy & - & $\mathrm{NMA} / \mathrm{HOAc}^{d}$ & 120 & 2 & 99 & 100 \\
\hline 13 & $\mathrm{Pd}(\mathrm{OAc})_{2}$ & bpy & - & NMA/HOAc ${ }^{d}$ & 100 & 10 & 87 & 100 \\
\hline 14 & $\mathrm{Pd}(\mathrm{OAc})_{2}$ & bpy & - & NMA/HOAc ${ }^{d}$ & 80 & 24 & 88 & 90 \\
\hline $15^{e}$ & $\mathrm{Pd}(\mathrm{OAc})_{2}$ & bpy & - & NMA $/ \mathrm{HOAc}^{d}$ & 120 & 2 & 98 & 100 \\
\hline $16^{e}$ & $\mathrm{Pd}(\mathrm{OAc})_{2}$ & bpy & - & HOAc & 120 & 24 & 44 & 99 \\
\hline $17^{e}$ & $\mathrm{Pd}(\mathrm{OAc})_{2}$ & - & - & $\mathrm{NMA} / \mathrm{HOAc}{ }^{d}$ & 120 & 30 & 19 & 23 \\
\hline 18 & - & - & - & $\mathrm{NMA} / \mathrm{HOAc}^{d}$ & 120 & 24 & nd & - \\
\hline
\end{tabular}

${ }^{a}$ Reactions were carried out using $1 \mathrm{a}(0.2 \mathrm{mmol})$, catalyst $(10 \mathrm{~mol} \%)$, ligand $(12 \mathrm{~mol} \%)$ in solvent $(\mathrm{c}=0.4 \mathrm{M}) .{ }^{b}$ Isolated yields. ${ }^{c}$ based on recovered starting material 1a. ${ }^{d}$ NMA $/ \mathrm{HOAc}=3 / 1 .{ }^{e}$ Reaction was performed with $\mathrm{Pd}(\mathrm{OAc})_{2}(5 \mathrm{~mol} \%)$, ligand $(6 \mathrm{~mol} \%)$. bpy: 2,2'-bipyridine; phen: 1,10-phenanthroline; D-CSA: D-(+)-camphorsulfonic acid; DMA: N,N-dimethylacetamide; NMA: $\mathrm{N}$-methylacetamide.

Treatment of substrate 1a with $10 \mathrm{~mol} \%$ of $\mathrm{Pd}(\mathrm{OAc})_{2}, 12 \mathrm{~mol} \%$ of 2,2'-bipyridine (bpy) and 1.5 equiv of D-(+)-camphorsulfonic acid (D-CSA) in NMA which was applicable to intermolecular variation except for the addition of $\mathrm{H}_{2} \mathrm{O}$, led a tandem cyclization which gave 4,5-dihydro-3aH-oxazolo[4,5-a]carbazol-2(10H)-one 2a in very low yield after 24 hours (Table S1, entry 1). To our delight, a 75\% yield of the desired product $2 \mathbf{a}$ was obtained in the absence of D-CSA (Table S1, entry 2). Notably, neither intermolecular or intramolcular cases of indoles $(\mathrm{C} 2$ ) $\mathrm{C}-\mathrm{H}$ addition to nitriles has been reported previously. The structure of the product $\mathbf{2 a}$ was confirmed by X-ray analysis. The influence of solvent on the reaction efficiency was investigated, and NMA gave superior result (Table S1, entries 2-8). $\mathrm{Pd}(\mathrm{TFA})_{2}$ and $\mathrm{Pd}\left(\mathrm{Ph}_{3} \mathrm{P}\right)_{2} \mathrm{Cl}_{2}$ were found to be inferior to $\mathrm{Pd}(\mathrm{OAc})_{2}$ (Table S1, entries 2, 9-10). Employment of other bidentate nitrogen ligand such as 1,10-phenanthroline (Phen) afforded the comparable yield (Table S1, entries 2, 11). Gaunt and co-workers disclosed that the 2-indolyl-palladium complex from the transfer of metal species was favorable in acidic medium (HOAc) in a Heck-type reaction. ${ }^{8}$ Delightfully, the use of NMA with the addition of HOAc as a co-solvent led to a dramatic acceleration on this reaction and provided the desired product 2a in almost quantitative yield (Table S1, entry 12). The decreasing temperatures led to the sluggish reactions (Table $1 \mathrm{~S}$, entries 13-14). Reducing the loading of $\mathrm{Pd}(\mathrm{OAc})_{2}$ and ligand to $5 \mathrm{~mol} \%$ and 6 
mol\% respectively, did not affect the efficiency of this cyclization (Table S1, entry 15). However, when HOAc was used as a sole solvent, the reaction proceeded sluggishly (Table S1, entry 16). Further investigation verified the necessity of Pd-catalyst and ligand, in which the absence of ligand resulted in a very low conversion, while no reaction occurred in the absence of both catalyst and ligand (Table S1, entries 17-18).

\section{General Procedure and Experimental Details of Palladium-Catalyzed Intramolecular Cyclization}

1) General Procedure and Experimental Details for the Preparation of Products 2, 4, 6 and 8 (Table 1, and Scheme 2)

A mixture of substrate $1(3,5$ or $7,0.2 \mathrm{mmol}), \mathrm{Pd}(\mathrm{OAc})_{2}(5 \mathrm{~mol} \%)$ and 2,2'-bipyridine $(6 \mathrm{~mol} \%)$ in HOAc/NMA (v/v $=1 / 3,0.5 \mathrm{~mL})$ was stirred at $120{ }^{\circ} \mathrm{C}$. Upon completion, the mixture was cooled to room temperature, and then $\mathrm{NaHCO}_{3}$ was added until no $\mathrm{CO}_{2}$ bubbles were generated. The resulting mixture was extracted with DCM three times. The combined organic layers were dried over $\mathrm{Na}_{2} \mathrm{SO}_{4}$, filtered, and concentrated under reduced pressure. The residue was purified by flash column chromatography on a silica gel using petroleum ether/EtOAc (10:1 to 5:1) as the eluent to give the desired product $2(\mathbf{4}, \mathbf{6}$ or $\mathbf{8})$.

3a,10-Dimethyl-3a,4,5,10-tetrahydro-2H-oxazolo[4,5-a]carbazol-2-one

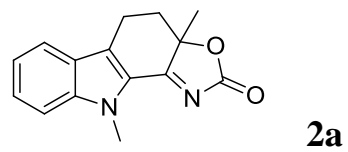

White solid (50.0 mg, 98\%), mp: 161.1-162.3 ${ }^{\circ} \mathrm{C} .{ }^{1} \mathrm{H}$ NMR (300 MHz, $\left.\mathrm{CDCl}_{3}\right) \delta 7.67-7.62(\mathrm{~m}, 1 \mathrm{H}), 7.53-7.43$ (m, 1H), $7.43-7.37(\mathrm{~m}, 1 \mathrm{H}), 7.24-7.17(\mathrm{~m}, 1 \mathrm{H}), 4.10(\mathrm{~s}, 3 \mathrm{H}), 3.30$ (ddd, $J=17.6,6.2,1.6 \mathrm{~Hz}, 1 \mathrm{H}), 3.07$ (ddd, $J$ $=17.1,11.5,5.2 \mathrm{~Hz}, 1 \mathrm{H}), 2.56(\mathrm{ddd}, J=12.5,5.2,1.6 \mathrm{~Hz}, 1 \mathrm{H}), 2.31-2.17(\mathrm{~m}, 1 \mathrm{H}), 1.62(\mathrm{~s}, 3 \mathrm{H}) .{ }^{13} \mathrm{C} \mathrm{NMR}(125$ $\left.\mathrm{MHz}, \mathrm{CDCl}_{3}\right) \delta 188.49,166.83,142.12,128.12,127.93,125.50,125.00,121.35,121.07,110.78,87.45,35.99$, 31.60, 23.09, 20.36. HRMS (ESI): calcd. for $\mathrm{C}_{15} \mathrm{H}_{15} \mathrm{~N}_{2} \mathrm{O}_{2}^{+}\left([\mathrm{M}+\mathrm{H}]^{+}\right)$: 255.1128, found: 255.1132 .

Gram scale: A mixture of substrate 1a $(1.32 \mathrm{~g}, 4.4 \mathrm{mmol}), \mathrm{Pd}(\mathrm{OAc})_{2}(5 \mathrm{~mol} \%)$ and 2,2'-bipyridine $(6 \mathrm{~mol} \%)$ in HOAc/NMA ( $/ / \mathrm{v}=1 / 3,11 \mathrm{~mL}$ ) was stirred at $120{ }^{\circ} \mathrm{C}$ for $4 \mathrm{~h}$. Then the mixture was cooled to room temperature, and then $\mathrm{NaHCO}_{3}$ was added. The resulting mixture was extracted with DCM three times. The combined organic layers were dried over $\mathrm{Na}_{2} \mathrm{SO}_{4}$, filtered, and concentrated under reduced pressure. The residue was purified by flash column chromatography on a silica gel using petroleum ether/EtOAc (10:1 to 5:1) as the eluent to give 2a in $95 \%$ yield (1.06 g).

\section{0-Benzyl-3a-methyl-3a,4,5,10-tetrahydro-2H-oxazolo[4,5-a]carbazol-2-one}

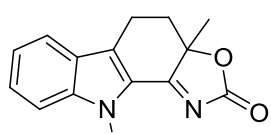

$\mathrm{Bn} \quad \mathbf{2 b}$

White solid (64.9 mg, 98\%), mp: 162.5-162.9 ${ }^{\circ} \mathrm{C} .{ }^{1} \mathrm{H}$ NMR (500 MHz, $\left.\mathrm{CDCl}_{3}\right) \delta 7.65(\mathrm{~d}, J=8.1 \mathrm{~Hz}, 1 \mathrm{H}), 7.43-$ $7.35(\mathrm{~m}, 2 \mathrm{H}), 7.27-7.16(\mathrm{~m}, 4 \mathrm{H}), 7.12(\mathrm{~d}, J=7.0 \mathrm{~Hz}, 2 \mathrm{H}), 5.97(\mathrm{~d}, J=16.1 \mathrm{~Hz}, 1 \mathrm{H}), 5.64(\mathrm{~d}, J=16.1 \mathrm{~Hz}, 1 \mathrm{H})$, $3.31(\mathrm{dd}, J=17.6,5.6 \mathrm{~Hz}, 1 \mathrm{H}), 3.08(\mathrm{ddd}, J=17.4,11.7,5.3 \mathrm{~Hz}, 1 \mathrm{H}), 2.56(\mathrm{dd}, J=12.5,4.7 \mathrm{~Hz}, 1 \mathrm{H}), 2.25(\mathrm{td}, J=$ 12.1, $6.2 \mathrm{~Hz}, 1 \mathrm{H}), 1.54$ (s, 3H). ${ }^{13} \mathrm{C}$ NMR $\left(125 \mathrm{MHz}, \mathrm{CDCl}_{3}\right) \delta 188.44,166.79,141.74,137.29,128.78,128.57$, $128.22,127.73,126.88,125.90,124.80,121.43,121.30,111.57,87.34,48.22,35.74,22.88,20.35$. HRMS (ESI): calcd. for $\mathrm{C}_{21} \mathrm{H}_{18} \mathrm{~N}_{2} \mathrm{O}_{2}\left([\mathrm{M}+\mathrm{H}]^{+}\right): 331.1441$, found: 331.1444 .

3a-Methyl-10-phenyl-4,5-dihydro-3aH-oxazolo[4,5-a]carbazol-2(10H)-one 


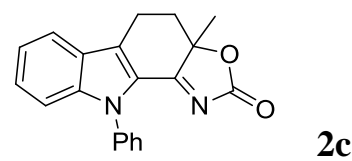

White solid (38.1 mg, 60\%), mp: 233.1-234.9 ${ }^{\circ} \mathrm{C} .{ }^{1} \mathrm{H}$ NMR $\left(500 \mathrm{MHz}, \mathrm{CDCl}_{3}\right) \delta 7.70(\mathrm{~d}, J=8.0 \mathrm{~Hz}, 1 \mathrm{H}), 7.53(\mathrm{t}, J$ $=7.4 \mathrm{~Hz}, 2 \mathrm{H}), 7.49-7.37(\mathrm{~m}, 4 \mathrm{H}), 7.34(\mathrm{~d}, J=8.4 \mathrm{~Hz}, 1 \mathrm{H}), 7.25(\mathrm{t}, J=7.1 \mathrm{~Hz}, 1 \mathrm{H}), 3.37(\mathrm{dd}, J=17.5,5.7 \mathrm{~Hz}$, $1 \mathrm{H}), 3.15-3.12(\mathrm{~m}, 1 \mathrm{H}), 2.59(\mathrm{dd}, J=12.4,4.7 \mathrm{~Hz}, 1 \mathrm{H}), 2.36-2.20(\mathrm{~m}, 1 \mathrm{H}), 1.65(\mathrm{~s}, 3 \mathrm{H}) .{ }^{13} \mathrm{C} \mathrm{NMR}(125 \mathrm{MHz}$, $\left.\mathrm{CDCl}_{3}\right) \delta 188.11,166.62,142.37,136.51,129.71,129.58,128.51,128.46,127.42,125.84,125.11,121.86,121.18$, 112.03, 87.20, 36.05, 22.72, 20.37. HRMS (ESI): calcd. for $\mathrm{C}_{20} \mathrm{H}_{16} \mathrm{~N}_{2} \mathrm{O}_{2}\left([\mathrm{M}+\mathrm{H}]^{+}\right): 317.1285$ found 317.1282 .

\section{3a-Methyl-3a,4,5,10-tetrahydro-2H-oxazolo[4,5-a]carbazol-2-one}

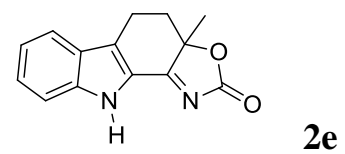

White solid (23.1 mg, 48\%), mp: 220.4-221.9 ${ }^{\circ} \mathrm{C} .{ }^{1} \mathrm{H}$ NMR $\left(500 \mathrm{MHz}, \mathrm{CDCl}_{3}\right) \delta 11.35(\mathrm{~s}, 1 \mathrm{H}), 7.88(\mathrm{~d}, J=8.5 \mathrm{~Hz}$, $1 \mathrm{H}), 7.62(\mathrm{~d}, J=8.1 \mathrm{~Hz}, 1 \mathrm{H}), 7.45(\mathrm{t}, J=7.5 \mathrm{~Hz}, 1 \mathrm{H}), 7.18(\mathrm{t}, J=7.5 \mathrm{~Hz}, 1 \mathrm{H}), 3.39-3.31(\mathrm{~m}, 1 \mathrm{H}), 3.19-3.08(\mathrm{~m}$, $1 \mathrm{H}), 2.67-2.58(\mathrm{~m}, 1 \mathrm{H}), 2.45-2.29(\mathrm{~m}, 1 \mathrm{H}), 1.69(\mathrm{~s}, 3 \mathrm{H}) .{ }^{13} \mathrm{C} \mathrm{NMR}\left(125 \mathrm{MHz}, \mathrm{CDCl}_{3}\right) \delta 188.17,166.38,141.79$, $130.50,128.87,126.00,123.59,121.37,120.80,114.45,87.29,36.54,23.37,20.59$. HRMS (ESI): calcd. for $\mathrm{C}_{14} \mathrm{H}_{12} \mathrm{~N}_{2} \mathrm{O}_{2}\left([\mathrm{M}+\mathrm{H}]^{+}\right): 241.0972$, found: 241.0977 .

\section{3a,6,10-Trimethyl-3a,4,5,10-tetrahydro-2H-oxazolo[4,5-a]carbazol-2-one}

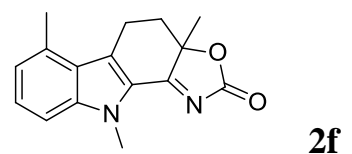

White solid (50.9 mg, 89\%), mp: $171.1-172.4{ }^{\circ} \mathrm{C} .{ }^{1} \mathrm{H}$ NMR $\left(500 \mathrm{MHz}, \mathrm{CDCl}_{3}\right) \delta 7.32(\mathrm{t}, J=7.7 \mathrm{~Hz}, 1 \mathrm{H}), 7.20(\mathrm{~d}, J$ $=8.5 \mathrm{~Hz}, 1 \mathrm{H}), 6.91(\mathrm{~d}, J=6.9 \mathrm{~Hz}, 1 \mathrm{H}), 4.07(\mathrm{~s}, 3 \mathrm{H}), 3.56(\mathrm{dd}, J=17.6,5.9 \mathrm{~Hz}, 1 \mathrm{H}), 3.33-3.22(\mathrm{~m}, 1 \mathrm{H}), 2.69(\mathrm{~s}$, $3 \mathrm{H}), 2.53(\mathrm{dd}, J=12.4,4.9 \mathrm{~Hz}, 1 \mathrm{H}), 2.21(\mathrm{td}, J=12.0,6.2 \mathrm{~Hz}, 1 \mathrm{H}), 1.60(\mathrm{~s}, 3 \mathrm{H}) .{ }^{13} \mathrm{C} \mathrm{NMR}\left(125 \mathrm{MHz} \mathrm{CDCl}_{3}\right) \delta$ $188.41,166.92,142.58,133.89,128.22,128.15,125.10,124.58,122.11,108.44,86.96,35.90,31.61,23.00,22.84$, 20.37. HRMS (ESI): calcd. for $\mathrm{C}_{16} \mathrm{H}_{16} \mathrm{~N}_{2} \mathrm{O}_{2}\left([\mathrm{M}+\mathrm{H}]^{+}\right)$: 269.1285, found 269.1288.

\section{7-Methoxy-3a,10-dimethyl-3a,4,5,10-tetrahydro-2H-oxazolo[4,5-a]carbazol-2-one}

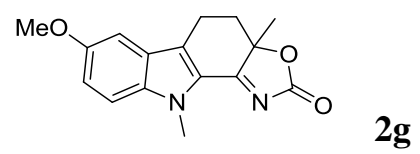

White solid (55.7 mg, 98\%), mp: $168.4-169.5{ }^{\circ} \mathrm{C} .{ }^{1} \mathrm{H}$ NMR $\left(500 \mathrm{MHz}, \mathrm{CDCl}_{3}\right) \delta 7.30(\mathrm{~d}, J=9.1 \mathrm{~Hz}, 1 \mathrm{H}), 7.14(\mathrm{dd}$, $J=9.1,2.4 \mathrm{~Hz}, 1 \mathrm{H}), 6.95(\mathrm{~d}, J=2.3 \mathrm{~Hz}, 1 \mathrm{H}), 4.07$ (s, 3H), 3.87 (s, 3H), 3.27-3.20 (m, 1H), 3.08-2.99 (m, 1H), 2.58-2.52 (m, 1H), 2.26-2.17 (m, 1H), $1.61(\mathrm{~s}, 3 \mathrm{H}) .{ }^{13} \mathrm{C} \mathrm{NMR}\left(125 \mathrm{MHz}, \mathrm{CDCl}_{3}\right) \delta 188.26,166.93,154.92,137.95$, $126.78,125.63,125.12,120.26,111.81,100.57,87.38,55.85,35.91,31.70,23.13,20.37$. HRMS (ESI): calcd. for $\mathrm{C}_{16} \mathrm{H}_{16} \mathrm{~N}_{2} \mathrm{O}_{3}\left([\mathrm{M}+\mathrm{H}]^{+}\right): 285.1234$, found 285.1225 .

\section{7-Bromo-3a,10-dimethyl-3a,4,5,10-tetrahydro-2H-oxazolo[4,5-a]carbazol-2-one}




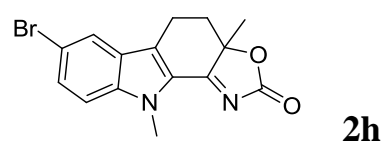

White solid (44.3 mg, 67\%), mp: 245.2 - $246.4{ }^{\circ} \mathrm{C} .{ }^{1} \mathrm{H}$ NMR $\left(500 \mathrm{MHz}, \mathrm{CDCl}_{3}\right) \delta 7.78(\mathrm{~s}, 1 \mathrm{H}), 7.53(\mathrm{~d}, J=8.9 \mathrm{~Hz}$, 1H), $7.30(\mathrm{~d}, J=8.9 \mathrm{~Hz}, 1 \mathrm{H}), 4.09$ (s, 3H), $3.23(\mathrm{dd}, J=17.5,5.9 \mathrm{~Hz}, 1 \mathrm{H}), 3.03$ (ddd, $J=17.2,11.7,5.1 \mathrm{~Hz}, 1 \mathrm{H})$, $2.57(\mathrm{dd}, J=12.5,5.0 \mathrm{~Hz}, 1 \mathrm{H}), 2.22(\mathrm{td}, J=12.1,6.1 \mathrm{~Hz}, 1 \mathrm{H}), 1.61(\mathrm{~s}, 3 \mathrm{H}) .{ }^{13} \mathrm{C} \mathrm{NMR}\left(125 \mathrm{MHz}, \mathrm{CDCl}_{3}\right) \delta 188.45$, $166.51,140.52,130.84,126.92,126.56,125.82,123.75,114.31,112.34,87.48,35.79,31.81,22.96,20.20$. HRMS (ESI): calcd. for $\mathrm{C}_{15} \mathrm{H}_{13} \mathrm{BrN}_{2} \mathrm{O}_{2}\left([\mathrm{M}+\mathrm{H}]^{+}\right)$: 333.0233, found 333.0229.

\section{7-Chloro-3a,10-dimethyl-3a,4,5,10-tetrahydro-2H-oxazolo[4,5-a]carbazol-2-one}

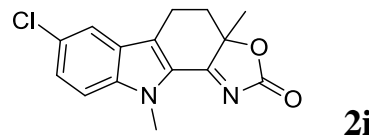

White solid (46.1 mg, 80\%), mp: 278.3-288.5 ${ }^{\circ} \mathrm{C} .{ }^{1} \mathrm{H}$ NMR (300 MHz, CDCl3) $\delta 7.61(\mathrm{~d}, J=1.7 \mathrm{~Hz}, 1 \mathrm{H}), 7.41(\mathrm{dd}$, $J=8.9,1.9 \mathrm{~Hz}, 1 \mathrm{H}), 7.34(\mathrm{~d}, J=8.9 \mathrm{~Hz}, 1 \mathrm{H}), 4.09(\mathrm{~s}, 3 \mathrm{H}), 3.30-3.16(\mathrm{~m}, 1 \mathrm{H}), 3.12-2.96(\mathrm{~m}, 1 \mathrm{H}), 2.62-2.51(\mathrm{~m}$, $1 \mathrm{H}), 2.28-2.15(\mathrm{~m}, 1 \mathrm{H}), 1.62(\mathrm{~s}, 3 \mathrm{H}) .{ }^{13} \mathrm{C}$ NMR $\left(125 \mathrm{MHz}, \mathrm{CDCl}_{3}\right) \delta 188.47,166.52,140.29,128.38,126.88$, 126.68, 126.23, 125.97, 120.50, 111.99, 87.47, 35.78, 31.81, 22.95, 20.19. HRMS (ESI): calcd. for $\mathrm{C}_{15} \mathrm{H}_{13} \mathrm{ClN}_{2} \mathrm{O}_{2}$ $\left([\mathrm{M}+\mathrm{H}]^{+}\right): 289.0738$, found 289.0740 .

8-Chloro-3a,10-dimethyl-3a,4,5,10-tetrahydro-2H-oxazolo[4,5-a]carbazol-2-one<smiles>Cn1c2c(c3ccc(Cl)cc31)CCC1(C)OC(=O)N=C21</smiles>

White solid (46.1 mg, 80\%), mp: 253.4-254.3 ${ }^{\circ} \mathrm{C} .{ }^{1} \mathrm{H}$ NMR $\left(500 \mathrm{MHz}, \mathrm{CDCl}_{3}\right) \delta 7.56(\mathrm{~d}, J=8.6 \mathrm{~Hz}, 1 \mathrm{H}), 7.40(\mathrm{~s}$, $1 \mathrm{H}), 7.16(\mathrm{~d}, J=8.6 \mathrm{~Hz}, 1 \mathrm{H}), 4.06(\mathrm{~s}, 3 \mathrm{H}), 3.26(\mathrm{dd}, J=17.5,5.9 \mathrm{~Hz}, 1 \mathrm{H}), 3.06(\mathrm{ddd}, J=17.3,11.8,5.2 \mathrm{~Hz}, 1 \mathrm{H})$, $2.56(\mathrm{dd}, J=12.5,5.1 \mathrm{~Hz}, 1 \mathrm{H}), 2.22(\mathrm{td}, J=12.1,6.1 \mathrm{~Hz}, 1 \mathrm{H}), 1.61(\mathrm{~s}, 3 \mathrm{H}) .{ }^{13} \mathrm{C} \mathrm{NMR}\left(125 \mathrm{MHz} \mathrm{CDCl}_{3}\right) \delta 188.26$, $166.59,142.28,134.23,127.81,125.64,124.04,122.34,122.19,110.70,87.36,35.83,31.75,23.02$, 20.27. HRMS (ESI): calcd. for $\mathrm{C}_{15} \mathrm{H}_{13} \mathrm{ClN}_{2} \mathrm{O}_{2}\left([\mathrm{M}+\mathrm{H}]^{+}\right): 289.0738$, found 289.0744 .

\section{3a,9,10-Trimethyl-3a,4,5,10-tetrahydro-2H-oxazolo[4,5-a]carbazol-2-one}

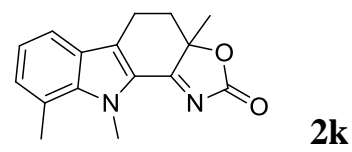

White solid (50.9 mg, 95\%), mp: 197.2-197.9 ${ }^{\circ} \mathrm{C} .{ }^{1} \mathrm{H}$ NMR $\left(500 \mathrm{MHz}, \mathrm{CDCl}_{3}\right) \delta 7.44(\mathrm{~d}, J=8.0 \mathrm{~Hz}, 1 \mathrm{H}), 7.14(\mathrm{~d}, J$ $=6.9 \mathrm{~Hz}, 1 \mathrm{H}), 7.04(\mathrm{t}, J=7.5 \mathrm{~Hz}, 1 \mathrm{H}), 4.38(\mathrm{~s}, 3 \mathrm{H}), 3.24(\mathrm{dd}, J=17.5,5.9 \mathrm{~Hz}, 1 \mathrm{H}), 3.03(\mathrm{ddd}, J=17.3,11.8,5.2$ $\mathrm{Hz}, 1 \mathrm{H}), 2.79(\mathrm{~s}, 3 \mathrm{H}), 2.54(\mathrm{dd}, J=12.4,4.9 \mathrm{~Hz}, 1 \mathrm{H}), 2.19(\mathrm{td}, J=12.1,6.1 \mathrm{~Hz}, 1 \mathrm{H}), 1.60(\mathrm{~s}, 3 \mathrm{H}) .{ }^{13} \mathrm{C} \mathrm{NMR}(125$ $\left.\mathrm{MHz}, \mathrm{CDCl}_{3}\right) \delta 188.45,166.75,141.35,130.44,127.99,126.38,125.35,122.92,121.16,119.20,87.57,35.77$, 34.68, 23.05, 20.14, 20.08. HRMS (ESI): calcd. for $\mathrm{C}_{16} \mathrm{H}_{16} \mathrm{~N}_{2} \mathrm{O}_{2}\left([\mathrm{M}+\mathrm{H}]^{+}\right)$: 269.1285, found 269.1287.

3a-Ethyl-10-methyl-3a,4,5,10-tetrahydro-2H-oxazolo[4,5-a]carbazol-2-one<smiles>CCC12CCc3c(n(C)c4ccccc34)C3=NC(=O)OC31CC2</smiles>

21

White solid (51.5 mg, 96\%), mp: $155.2-155.9{ }^{\circ} \mathrm{C} .{ }^{1} \mathrm{H}$ NMR $\left(500 \mathrm{MHz}, \mathrm{CDCl}_{3}\right) \delta 7.63(\mathrm{~d}, J=7.8 \mathrm{~Hz}, 1 \mathrm{H}), 7.49-$ 
$7.43(\mathrm{~m}, 1 \mathrm{H}), 7.39(\mathrm{~d}, J=8.2 \mathrm{~Hz}, 1 \mathrm{H}), 7.19(\mathrm{t}, J=7.1 \mathrm{~Hz}, 1 \mathrm{H}), 4.08(\mathrm{~s}, 3 \mathrm{H}), 3.31-3.19(\mathrm{~m}, 1 \mathrm{H}), 3.14-3.00(\mathrm{~m}$, $1 \mathrm{H}), 2.66-2.58(\mathrm{~m}, 1 \mathrm{H}), 2.25-2.15(\mathrm{~m}, 1 \mathrm{H}), 2.06-1.95(\mathrm{~m}, 1 \mathrm{H}), 1.93-1.80(\mathrm{~m}, 1 \mathrm{H}), 0.95(\mathrm{t}, J=6.9 \mathrm{~Hz}, 3 \mathrm{H})$. ${ }^{13} \mathrm{C}$ NMR $\left(125 \mathrm{MHz}, \mathrm{CDCl}_{3}\right) \delta 187.88,167.12,142.01,127.99,127.92,125.49,125.16,121.28,120.99,110.71$, 89.96, 33.99, 31.49, 28.62, 20.07, 7.84. HRMS (ESI): calcd. for $\mathrm{C}_{16} \mathrm{H}_{16} \mathrm{~N}_{2} \mathrm{O}_{2}\left([\mathrm{M}+\mathrm{H}]^{+}\right)$: 269.1285, found: 269.1286 .

\section{0-Methyl-3a-phenyl-3a,4,5,10-tetrahydro-2H-oxazolo[4,5-a]carbazol-2-one}

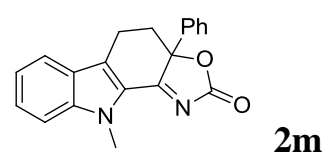

White solid (61.9 mg, 98\%), mp: 183.3-184.5 ${ }^{\circ} \mathrm{C} .{ }^{1} \mathrm{H}$ NMR (500 MHz, $\left.\mathrm{CDCl}_{3}\right) \delta 7.57(\mathrm{~d}, J=8.1 \mathrm{~Hz}, 1 \mathrm{H}), 7.51-$ $7.41(\mathrm{~m}, 2 \mathrm{H}), 7.38-7.34(\mathrm{~m}, 2 \mathrm{H}), 7.33-7.28(\mathrm{~m}, 3 \mathrm{H}), 7.17(\mathrm{t}, J=7.3 \mathrm{~Hz}, 1 \mathrm{H}), 4.20(\mathrm{~s}, 3 \mathrm{H}), 3.21(\mathrm{dd}, J=17.1,3.8$ $\mathrm{Hz}, 1 \mathrm{H}), 3.01(\mathrm{dd}, J=12.3,2.7 \mathrm{~Hz}, 1 \mathrm{H}), 2.72-2.58(\mathrm{~m}, 1 \mathrm{H}), 2.47(\mathrm{td}, J=12.1,5.2 \mathrm{~Hz}, 1 \mathrm{H}) .{ }^{13} \mathrm{C}$ NMR $(125 \mathrm{MHz}$, $\left.\mathrm{CDCl}_{3}\right) \delta 185.93,166.34,142.26,136.36,129.62,129.38,128.90,128.31,126.51,126.26,125.51,121.47,121.12$, 110.85, 90.61, 37.35, 31.76, 20.09. HRMS (ESI): calcd. for $\mathrm{C}_{20} \mathrm{H}_{16} \mathrm{~N}_{2} \mathrm{O}_{2}\left([\mathrm{M}+\mathrm{H}]^{+}\right)$: 317.1285, found 317.1291.

3a-(4-Chlorophenyl)-10-methyl-3a,4,5,10-tetrahydro-2H-oxazolo[4,5-a]carbazol-2-one

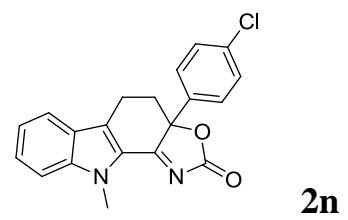

White solid (61.4 mg, 90\%), mp: 182.2-183.6 ${ }^{\circ} \mathrm{C} .{ }^{1} \mathrm{H}$ NMR (500 MHz, $\left.\mathrm{CDCl}_{3}\right) \delta 7.58(\mathrm{~d}, J=8.1 \mathrm{~Hz}, 1 \mathrm{H}), 7.51-$ $7.47(\mathrm{~m}, 1 \mathrm{H}), 7.44(\mathrm{~d}, J=8.5 \mathrm{~Hz}, 1 \mathrm{H}), 7.33-7.26(\mathrm{~m}, 4 \mathrm{H}), 7.19(\mathrm{t}, J=7.4 \mathrm{~Hz}, 1 \mathrm{H}), 4.21(\mathrm{~s}, 3 \mathrm{H}), 3.23(\mathrm{dd}, J=17.2$, $3.9 \mathrm{~Hz}, 1 \mathrm{H}), 2.94(\mathrm{dd}, J=12.4,2.9 \mathrm{~Hz}, 1 \mathrm{H}), 2.67-2.59(\mathrm{~m}, 1 \mathrm{H}), 2.53-2.46(\mathrm{~m}, 1 \mathrm{H}) .{ }^{13} \mathrm{C} \mathrm{NMR}\left(125 \mathrm{MHz}, \mathrm{CDCl}_{3}\right)$ $\delta 185.29,166.11,142.37,135.45,134.96,129.72,129.14,128.92,128.57,127.70,126.27,125.49,121.53,121.29$, 110.93, 89.96, 37.44, 31.84, 20.03. HRMS (ESI): calcd. for $\mathrm{C}_{20} \mathrm{H}_{15} \mathrm{ClN}_{2} \mathrm{O}_{2}\left([\mathrm{M}+\mathrm{H}]^{+}\right): 351.0895$, found 351.0887 .

\section{3a-(3, 4-Dichlorophenyl)-10-methyl-3a,4,5,10-tetrahydro-2H-oxazolo[4,5-a]carbazol-2-one}

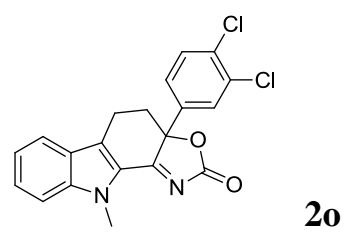

White solid (72.0 mg, 96\%), mp: 207.3-208.4 ${ }^{\circ} \mathrm{C} .{ }^{1} \mathrm{H}$ NMR $(500 \mathrm{MHz}, \mathrm{CDCl} 3) \delta 7.59(\mathrm{~d}, J=8.1 \mathrm{~Hz}, 1 \mathrm{H})$, 7.53-7.48 (m, 2H), 7.45 (d, $J=8.4 \mathrm{~Hz}, 1 \mathrm{H}), 7.37$ (d, $J=8.4 \mathrm{~Hz}, 1 \mathrm{H}), 7.22-7.14$ (m, 2H), 4.20 (s, 3H), 3.26 (dd, $J$ $=17.3,4.1 \mathrm{~Hz}, 1 \mathrm{H}), 2.92(\mathrm{dd}, J=12.5,2.9 \mathrm{~Hz}, 1 \mathrm{H}), 2.70-2.61(\mathrm{~m}, 1 \mathrm{H}), 2.54-2.40(\mathrm{~m}, 1 \mathrm{H}) .{ }^{13} \mathrm{C} \mathrm{NMR}(125 \mathrm{MHz}$, $\mathrm{CDCl} 3) \delta 184.52,165.81,142.47,136.55,133.79,133.34,130.76,129.94,128.78,128.48,125.95,125.47,125.43$, $121.55,121.37,110.96,89.30,37.32,31.84$, 19.94. HRMS (ESI): calcd. for $\mathrm{C}_{20} \mathrm{H}_{14} \mathrm{Cl}_{2} \mathrm{~N}_{2} \mathrm{O}_{2}\left([\mathrm{M}+\mathrm{H}]^{+}\right): 385.0505$, found 385.0501 .

\section{3a,6-Dimethyl-3a,4,5,6-tetrahydro-2H-oxazolo[5,4-c]carbazol-2-one}

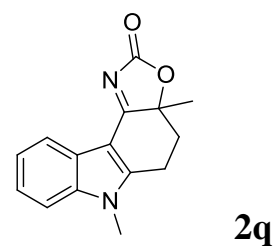


White solid (42.1 mg, 83\%), mp: 156.6-157.3 ${ }^{\circ} \mathrm{C} .{ }^{1} \mathrm{H}$ NMR (300 MHz, $\left.\mathrm{CDCl}_{3}\right) \delta 8.11-8.06(\mathrm{~m}, 1 \mathrm{H}), 7.37-7.31$ $(\mathrm{m}, 3 \mathrm{H}), 3.78(\mathrm{~s}, 3 \mathrm{H}), 3.20-3.00(\mathrm{~m}, 2 \mathrm{H}), 2.61-2.52(\mathrm{~m}, 1 \mathrm{H}), 2.27-2.17(\mathrm{~m}, 1 \mathrm{H}), 1.56(\mathrm{~s}, 3 \mathrm{H}) .{ }^{13} \mathrm{C} \mathrm{NMR}(125$ $\left.\mathrm{MHz}, \mathrm{CDCl}_{3}\right) \delta 191.38,168.01,149.42,139.03,124.22,123.71,123.37,121.32,110.13,105.19,84.94,33.86$, 30.58, 23.43, 21.31. HRMS (ESI): calcd. for $\mathrm{C}_{15} \mathrm{H}_{14} \mathrm{~N}_{2} \mathrm{O}_{2}\left([\mathrm{M}+\mathrm{H}]^{+}\right): 255.1128$, found 255.1121 .

\section{6-Methyl-3a-phenyl-3a,4,5,6-tetrahydro-2H-oxazolo[5,4-c]carbazol-2-one}<smiles>Cn1c2c(c3ccccc31)CC1OC(=O)N=C1c1ccccc1-2</smiles>

White solid (54.4 mg, 86\%), mp: 247.3-248.6 ${ }^{\circ} \mathrm{C} .{ }^{1} \mathrm{H}$ NMR $(500 \mathrm{MHz}, \mathrm{CDCl} 3) \delta 8.18(\mathrm{~d}, J=5.7 \mathrm{~Hz}, 1 \mathrm{H})$, 7.40-7.34 (m, 3H), $7.31(\mathrm{~d}, J=7.4 \mathrm{~Hz}, 2 \mathrm{H}), 7.29-7.22(\mathrm{~m}, 3 \mathrm{H}), 3.67(\mathrm{~s}, 3 \mathrm{H}), 3.10-2.95(\mathrm{~m}, 2 \mathrm{H}), 2.63-2.53(\mathrm{~m}, 1 \mathrm{H})$, 2.43-2.34 (m, 1H). ${ }^{13} \mathrm{C}$ NMR $\left(125 \mathrm{MHz}, \mathrm{CDCl}_{3}\right) \delta 188.66,167.59,150.66,139.14,136.82,129.26,128.85,126.42$, 124.44, 123.64, 123.54, 121.60, 110.26, 107.17, 88.36, 35.08, 30.61, 21.19. HRMS (ESI): calcd. for $\mathrm{C}_{20} \mathrm{H}_{16} \mathrm{~N}_{2} \mathrm{O}_{2}$ $\left([\mathrm{M}+\mathrm{H}]^{+}\right): 317.1285$, found 317.1288 .

\section{3a-(4-Chlorophenyl)-6-methyl-3a,4,5,6-tetrahydro-2H-oxazolo[5,4-c]carbazol-2-one}

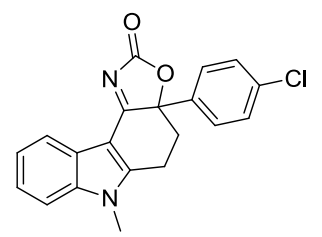

\section{$2 \mathrm{~s}$}

White solid (62.3 mg, 89\%), mp: 162.5-162.9 ${ }^{\circ} \mathrm{C} .{ }^{1} \mathrm{H}$ NMR (500 MHz, $\left.\mathrm{CDCl}_{3}\right) \delta 8.16$ (brs, 1H), 7.38 (brs, 3H), $7.32-7.14(\mathrm{~m}, 4 \mathrm{H}), 3.71(\mathrm{~s}, 3 \mathrm{H}), 3.15-3.02(\mathrm{~m}, 1 \mathrm{H}), 3.00-2.87(\mathrm{~m}, 1 \mathrm{H}), 2.64-2.52(\mathrm{~m}, 1 \mathrm{H}), 2.50-2.34(\mathrm{~m}$, 1H). ${ }^{13} \mathrm{C}$ NMR $\left(125 \mathrm{MHz}, \mathrm{CDCl}_{3}\right) \delta 188.04,167.32,150.51,139.18,135.45,135.28,129.07,127.92,124.64$, $123.81,123.47,121.66,110.33,107.08,87.66,35.13,30.69,21.13$. HRMS (ESI): calcd. for $\mathrm{C}_{20} \mathrm{H}_{15} \mathrm{ClN}_{2} \mathrm{O}_{2}$ $\left([\mathrm{M}+\mathrm{H}]^{+}\right): 351.0895$, found 351.0900 .

\section{3a-Methyl-4,5-dihydro-3aH-oxazolo[5,4-c]carbazol-2(6H)-one}

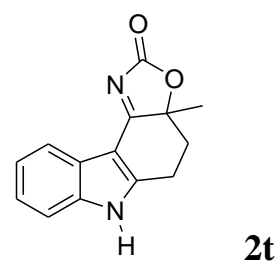

White solid (32.2 mg, 67\%), mp: 259.1-259.8 ${ }^{\circ} \mathrm{C} .{ }^{1} \mathrm{H}$ NMR (500 MHz, DMSO) $\delta 12.53(\mathrm{~s}, 1 \mathrm{H}), 7.82-7.77$ (m, 1H), $7.52-7.46(\mathrm{~m}, 1 \mathrm{H}), 7.31-7.23(\mathrm{~m}, 2 \mathrm{H}), 3.29-3.21(\mathrm{~m}, 1 \mathrm{H}), 3.18-3.12(\mathrm{~m}, 1 \mathrm{H}), 2.47(\mathrm{dd}, J=12.3,4.5 \mathrm{~Hz}$, $1 \mathrm{H}), 2.22-2.14(\mathrm{~m}, 1 \mathrm{H}), 1.51(\mathrm{~s}, 3 \mathrm{H}) .{ }^{13} \mathrm{C}$ NMR (125 MHz, DMSO) $\delta 191.60,167.07,151.45,137.72,123.69$, $123.65,122.43,120.03,112.38,104.33,84.88,34.08,23.26,21.63$. HRMS (ESI): calcd. for $\mathrm{C}_{14} \mathrm{H}_{12} \mathrm{~N}_{2} \mathrm{O}_{2}\left([\mathrm{M}+\mathrm{H}]^{+}\right)$: 241.0972 , found 241.0966 .

\section{3a-Methyl-4,5-dihydrooxazolo[4',5':3,4]pyrido[1,2-a]indol-2(3aH)-one}<smiles>CC12CCn3c(cc4ccccc43)C1=NC(=O)O2</smiles> 
White solid (28.8 mg, 60\%), mp: 179.4-180.2 ${ }^{\circ} \mathrm{C} .{ }^{1} \mathrm{H}$ NMR (500 MHz, DMSO) $\delta 7.80(\mathrm{~d}, J=8.0 \mathrm{~Hz}, 1 \mathrm{H}), 7.60(\mathrm{~d}$, $J=8.3 \mathrm{~Hz}, 1 \mathrm{H}), 7.51(\mathrm{~s}, 1 \mathrm{H}), 7.44(\mathrm{t}, J=7.5 \mathrm{~Hz}, 1 \mathrm{H}), 7.22(\mathrm{t}, J=7.4 \mathrm{~Hz}, 1 \mathrm{H}), 4.59(\mathrm{dd}, J=12.7,6.3 \mathrm{~Hz}, 1 \mathrm{H}), 4.31$ $(\operatorname{td}, J=12.5,4.2 \mathrm{~Hz}, 1 \mathrm{H}), 2.71(\mathrm{dd}, J=12.3,3.7 \mathrm{~Hz}, 1 \mathrm{H}), 2.48-2.40(\mathrm{~m}, 1 \mathrm{H}), 1.60(\mathrm{~s}, 3 \mathrm{H}) .{ }^{13} \mathrm{C} \mathrm{NMR}(125 \mathrm{MHz}$, DMSO) $\delta 189.56,165.77,138.93,127.57,126.44,125.69,123.02,121.67,111.54,107.33,85.11,40.29,32.32$, 21.37. HRMS (ESI): calcd. for $\mathrm{C}_{14} \mathrm{H}_{12} \mathrm{~N}_{2} \mathrm{O}_{2}\left([\mathrm{M}+\mathrm{H}]^{+}\right)$: 241.0972, found 241.0975.

\section{3a,11-Dimethyl-4,5-dihydrooxazolo[4',5':3,4]pyrido[1,2-a]indol-2(3aH)-one}

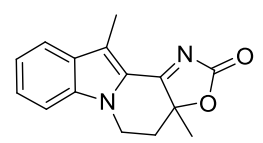

$2 \mathbf{v}$

White solid (48.8 mg, 96\%), mp: 270.8-271.4 ${ }^{\circ} \mathrm{C} .{ }^{1} \mathrm{H}$ NMR (500 MHz, $\left.\mathrm{CDCl}_{3}\right) \delta 7.72(\mathrm{~d}, J=8.1 \mathrm{~Hz}, 1 \mathrm{H}), 7.44(\mathrm{t}, J$ $=7.6 \mathrm{~Hz}, 1 \mathrm{H}), 7.34(\mathrm{~d}, J=8.4 \mathrm{~Hz}, 1 \mathrm{H}), 7.21(\mathrm{t}, J=7.5 \mathrm{~Hz}, 1 \mathrm{H}), 4.45(\mathrm{dd}, J=12.5,6.8 \mathrm{~Hz}, 1 \mathrm{H}), 4.22(\mathrm{td}, J=12.6$, $4.9 \mathrm{~Hz}, 1 \mathrm{H}), 2.73(\mathrm{~s}, 3 \mathrm{H}), 2.67(\mathrm{dd}, J=12.8,4.8 \mathrm{~Hz}, 1 \mathrm{H}), 2.40-2.31(\mathrm{~m}, 1 \mathrm{H}), 1.63(\mathrm{~s}, 3 \mathrm{H}) .{ }^{13} \mathrm{C} \mathrm{NMR}(125 \mathrm{MHz}$, $\left.\mathrm{CDCl}_{3}\right) \delta 188.94,166.85,139.08,128.61,127.60,123.44,123.07,121.83,121.26,110.30,84.78,40.21,33.05$, 22.22, 10.28. HRMS (ESI): calcd. for $\mathrm{C}_{15} \mathrm{H}_{14} \mathrm{~N}_{2} \mathrm{O}_{2}\left([\mathrm{M}+\mathrm{H}]^{+}\right)$: 255.1128 , found 255.1133 .

\section{Methyl 2-(3a-methyl-2-oxo-2,3a,4,5-tetrahydrooxazolo[4',5':3,4]pyrido[1,2-a]indol-11-yl) acetate}

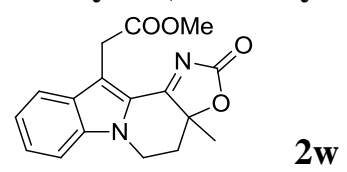

White solid (41.8 mg, 67\%), mp: 216.9-217.3 ${ }^{\circ} \mathrm{C} .{ }^{1} \mathrm{H}$ NMR (500 MHz, CDCl3) $\delta 7.72(\mathrm{~d}, J=8.0 \mathrm{~Hz}, 1 \mathrm{H}), 7.46$ (t, $J$ $=7.4 \mathrm{~Hz}, 1 \mathrm{H}), 7.37(\mathrm{~d}, J=8.2 \mathrm{~Hz}, 1 \mathrm{H}), 7.27-7.21(\mathrm{~m}, 1 \mathrm{H}), 4.49(\mathrm{dd}, J=12.1,6.6 \mathrm{~Hz}, 1 \mathrm{H}), 4.40(\mathrm{~d}, J=16.8 \mathrm{~Hz}$, $1 \mathrm{H}), 4.29-4.20(\mathrm{~m}, 2 \mathrm{H}), 3.71(\mathrm{~s}, 3 \mathrm{H}), 2.71-2.68(\mathrm{~m}, 1 \mathrm{H}), 2.44-2.34(\mathrm{~m}, 1 \mathrm{H}), 1.65(\mathrm{~s}, 3 \mathrm{H}) .{ }^{13} \mathrm{C} \mathrm{NMR}(125 \mathrm{MHz}$, $\left.\mathrm{CDCl}_{3}\right) \delta 188.91,170.96,166.55,138.92,128.14,127.57,123.86,122.03,121.84,117.78,110.53,84.83,52.37$, 40.36, 33.01, 30.44, 22.05. HRMS (ESI): calcd. for $\mathrm{C}_{17} \mathrm{H}_{16} \mathrm{~N}_{2} \mathrm{O}_{4}\left([\mathrm{M}+\mathrm{H}]^{+}\right)$: 313.1183 , found 313.1180.

\section{1-Methyl-3a-phenyl-4,5-dihydrooxazolo[4',5' :3,4]pyrido[1,2-a]indol-2(3aH)-one}

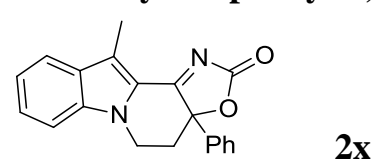

White solid (61.3 mg, 97\%), mp: 191.9-192.6 ${ }^{\circ} \mathrm{C} .{ }^{1} \mathrm{H} \mathrm{NMR}\left(500 \mathrm{MHz}, \mathrm{CDCl}_{3}\right) \delta 7.77(\mathrm{~d}, J=8.1 \mathrm{~Hz}, 1 \mathrm{H}), 7.41(\mathrm{t}, J$ $=7.6 \mathrm{~Hz}, 1 \mathrm{H}), 7.36-7.28(\mathrm{~m}, 3 \mathrm{H}), 7.25-7.19(\mathrm{~m}, 4 \mathrm{H}), 4.38(\mathrm{dd}, J=12.3,5.6 \mathrm{~Hz}, 1 \mathrm{H}), 3.72(\mathrm{td}, J=12.5,4.1 \mathrm{~Hz}$, $1 \mathrm{H}), 3.07(\mathrm{dd}, J=12.9,3.3 \mathrm{~Hz}, 1 \mathrm{H}), 2.86(\mathrm{~s}, 3 \mathrm{H}), 2.61(\mathrm{td}, J=12.8,6.0 \mathrm{~Hz}, 1 \mathrm{H}) .{ }^{13} \mathrm{C} \mathrm{NMR}\left(125 \mathrm{MHz}, \mathrm{CDCl}_{3}\right) \delta$ $186.38,166.45,139.23,135.50,129.72,129.27,128.69,127.68,126.33,124.47,123.25,121.99,121.34,110.40$, 88.45, 40.00, 34.34, 10.50. HRMS (ESI): calcd. for $\mathrm{C}_{20} \mathrm{H}_{16} \mathrm{~N}_{2} \mathrm{O}_{2}\left([\mathrm{M}+\mathrm{H}]^{+}\right)$: 317.1285 , found 317.1287.

\section{3a-Methyl-11-(3-oxobutyl)-4,5-dihydrooxazolo[4',5':3,4]pyrido[1,2-a]indol-2(3aH)-one}

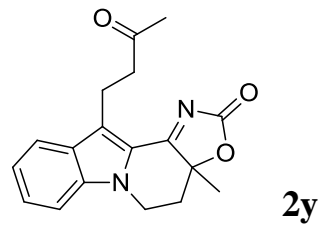

White solid (50.1 mg, 82\%), mp: 193.2-194.1 ${ }^{\circ} \mathrm{C} .{ }^{1} \mathrm{H}$ NMR (500 MHz, $\left.\mathrm{CDCl}_{3}\right) \delta 7.81(\mathrm{~d}, J=8.1 \mathrm{~Hz}, 1 \mathrm{H}), 7.45(\mathrm{t}, J$ $=7.6 \mathrm{~Hz}, 1 \mathrm{H}), 7.35(\mathrm{~d}, J=8.4 \mathrm{~Hz}, 1 \mathrm{H}), 7.23(\mathrm{t}, J=7.5 \mathrm{~Hz}, 1 \mathrm{H}), 4.50-4.45(\mathrm{~m}, 1 \mathrm{H}), 4.26-4.20(\mathrm{~m}, 1 \mathrm{H}), 3.53-$ 
$3.48(\mathrm{~m}, 1 \mathrm{H}), 3.40-3.32(\mathrm{~m}, 1 \mathrm{H}), 3.02-2.94(\mathrm{~m}, 1 \mathrm{H}), 2.90-2.82(\mathrm{~m}, 1 \mathrm{H}), 2.70(\mathrm{dd}, J=12.8,4.6 \mathrm{~Hz}, 1 \mathrm{H}), 2.42-$ $2.36(\mathrm{~m}, 1 \mathrm{H}), 2.14(\mathrm{~s}, 3 \mathrm{H}), 1.64(\mathrm{~s}, 3 \mathrm{H}) .{ }^{13} \mathrm{C} \mathrm{NMR}\left(125 \mathrm{MHz}, \mathrm{CDCl}_{3}\right) \delta 207.83,188.63,166.70,139.04,127.83$, $127.65,126.36,123.01,122.01,121.57,110.38,84.74,43.74,40.28,33.02,30.00,22.17,19.06$. HRMS (ESI): calcd. for $\mathrm{C}_{18} \mathrm{H}_{18} \mathrm{~N}_{2} \mathrm{O}_{3}\left([\mathrm{M}+\mathrm{H}]^{+}\right): 311.1390$, found 311.1393.

\section{1-Methyl-3a-phenyl-3a,4,5,6-tetrahydrooxazolo[5',4':6,7]cyclohepta[1,2-b]indol-2(11H)-one}

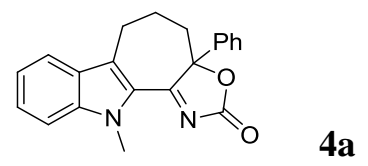

White solid (65.4 mg, 99\%), mp: 187.5-188.6 ${ }^{\circ} \mathrm{C} .{ }^{1} \mathrm{H}$ NMR (500 MHz, $\left.\mathrm{CDCl}_{3}\right) \delta 7.61(\mathrm{~d}, J=8.1 \mathrm{~Hz}, 1 \mathrm{H}), 7.51-$ $7.41(\mathrm{~m}, 2 \mathrm{H}), 7.31(\mathrm{~s}, 5 \mathrm{H}), 7.17(\mathrm{t}, J=7.3 \mathrm{~Hz}, 1 \mathrm{H}), 4.19(\mathrm{~s}, 3 \mathrm{H}), 2.97-3.03(\mathrm{~m}, 2 \mathrm{H}), 2.91-2.83(\mathrm{~m}, 1 \mathrm{H}), 2.32-$ $2.24(\mathrm{~m}, 1 \mathrm{H}), 2.02-1.93(\mathrm{~m}, 2 \mathrm{H}) .{ }^{13} \mathrm{C}$ NMR $\left(125 \mathrm{MHz}, \mathrm{CDCl}_{3}\right) \delta 188.10,165.79,141.39,137.49,129.29,129.23$, $129.03,127.89,127.16,126.85,126.64,121.00,120.77,110.80,93.87,34.95,32.85,24.21,21.42$. HRMS (ESI): calcd. for $\mathrm{C}_{21} \mathrm{H}_{18} \mathrm{~N}_{2} \mathrm{O}_{2}\left([\mathrm{M}+\mathrm{H}]^{+}\right): 331.1441$, found 331.1430 .

\section{3a,12-Dimethyl-3a,4,5,6,7,12-hexahydro-2H-oxazolo[5',4':7,8]cycloocta[1,2-b]indol-2-one}

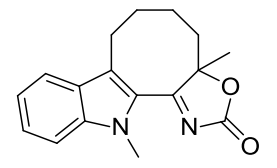

4b

White solid (38.4 mg, 68\%), mp: 196.2-197.4 ${ }^{\circ} \mathrm{C} .{ }^{1} \mathrm{H}$ NMR (500 MHz, $\left.\mathrm{CDCl}_{3}\right) \delta 7.70(\mathrm{~d}, J=8.1 \mathrm{~Hz}, 1 \mathrm{H}), 7.48-$ $7.37(\mathrm{~m}, 2 \mathrm{H}), 7.19(\mathrm{t}, J=7.3 \mathrm{~Hz}, 1 \mathrm{H}), 4.07(\mathrm{~s}, 3 \mathrm{H}), 3.38-3.31(\mathrm{~m}, 1 \mathrm{H}), 2.93-2.82(\mathrm{~m}, 1 \mathrm{H}), 2.48(\mathrm{dd}, J=15.6$, $8.2 \mathrm{~Hz}, 1 \mathrm{H}), 2.09(\mathrm{dd}, J=15.4,11.7 \mathrm{~Hz}, 1 \mathrm{H}), 1.95-1.85(\mathrm{~m}, 1 \mathrm{H}), 1.83-1.74(\mathrm{~m}, 1 \mathrm{H}), 1.70(\mathrm{~s}, 3 \mathrm{H}), 1.68-1.60$ $(\mathrm{m}, 1 \mathrm{H}), 1.58-1.46(\mathrm{~m}, 1 \mathrm{H}) .{ }^{13} \mathrm{C}$ NMR $\left(125 \mathrm{MHz}, \mathrm{CDCl}_{3}\right) \delta 190.60,165.77,140.82,127.28,126.91,126.14$, 120.62, 120.51, 110.69, 93.99, 34.84, 33.48, 27.28, 26.72, 22.49, 21.68. HRMS (ESI): calcd. for $\mathrm{C}_{17} \mathrm{H}_{18} \mathrm{~N}_{2} \mathrm{O}_{2}$ $\left([\mathrm{M}+\mathrm{H}]^{+}\right): 283.1441$, found 283.1447 .

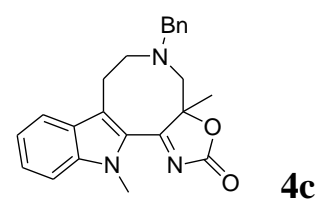

White solid (29.8 mg, 40\%), mp: 156.9-157.6 ${ }^{\circ} \mathrm{C} .{ }^{1} \mathrm{H}$ NMR $\left(500 \mathrm{MHz}, \mathrm{CDCl}_{3}\right) \delta 7.52(\mathrm{~d}, J=7.9 \mathrm{~Hz}, 1 \mathrm{H})$, 7.38-7.30 (m, 5H), 7.27-7.25 (m, 2H), $7.17(\mathrm{t}, J=7.4 \mathrm{~Hz}, 1 \mathrm{H}), 5.17(\mathrm{~d}, J=14.1 \mathrm{~Hz}, 1 \mathrm{H}), 4.27(\mathrm{~d}, J=14.2 \mathrm{~Hz}, 1 \mathrm{H})$, $4.14(\mathrm{~d}, J=13.6 \mathrm{~Hz}, 1 \mathrm{H}), 3.79(\mathrm{~d}, J=14.3 \mathrm{~Hz}, 1 \mathrm{H}), 3.67(\mathrm{~s}, 3 \mathrm{H}), 3.21-3.17(\mathrm{~m}, 1 \mathrm{H}), 2.89-2.85(\mathrm{~m}, 1 \mathrm{H}), 2.81-2.71$ $(\mathrm{m}, 1 \mathrm{H}), 2.70-2.59(\mathrm{~m}, 1 \mathrm{H}), 2.22(\mathrm{~s}, 3 \mathrm{H}) .{ }^{13} \mathrm{C} \mathrm{NMR}\left(125 \mathrm{MHz}, \mathrm{CDCl}_{3}\right) \delta 156.29,138.81,138.10,134.49,128.84$, $128.45,127.22$, 127.07, 123.29, 120.02, 118.80, 117.51, 109.99, 62.85, 30.90, 29.82, 11.94. HRMS (ESI): calcd. for $\mathrm{C}_{23} \mathrm{H}_{23} \mathrm{~N}_{3} \mathrm{O}_{2}\left([\mathrm{M}+\mathrm{H}]^{+}\right): 374.1863$, found 374.1868 .

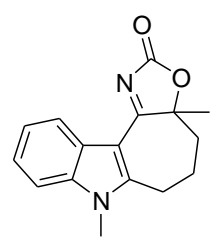

6a 
White solid (42.9 mg, 80\%), mp: 215.2-216.3 ${ }^{\circ} \mathrm{C} .{ }^{1} \mathrm{H}$ NMR (500 MHz, $\left.\mathrm{CDCl}_{3}\right) \delta 8.36(\mathrm{~d}, J=5.2 \mathrm{~Hz}, 1 \mathrm{H}), 7.34-$ $7.28(\mathrm{~m}, 3 \mathrm{H}), 3.77(\mathrm{~s}, 3 \mathrm{H}), 3.14-3.01(\mathrm{~m}, 2 \mathrm{H}), 2.37-2.31(\mathrm{~m}, 1 \mathrm{H}), 2.27-2.18(\mathrm{~m}, 1 \mathrm{H}), 2.16-2.10(\mathrm{~m}, 1 \mathrm{H}), 2.09$ $-2.00(\mathrm{~m}, 1 \mathrm{H}), 1.56(\mathrm{~s}, 3 \mathrm{H}) .{ }^{13} \mathrm{C}$ NMR $\left(125 \mathrm{MHz}, \mathrm{CDCl}_{3}\right) \delta 192.78,167.69,149.67,137.87,126.25,123.98$, 123.27, 122.46, 109.69, 105.88, 89.56, 35.29, 30.54, 28.12, 26.37, 22.65. HRMS (ESI): calcd. for $\mathrm{C}_{16} \mathrm{H}_{16} \mathrm{~N}_{2} \mathrm{O}_{2}$ $\left([\mathrm{M}+\mathrm{H}]^{+}\right): 269.1285$, found 269.1284 .

\section{3a,8-Dimethyl-3a,4,5,6,7,8-hexahydro-2H-oxazolo[4',5' :3,4]cycloocta[1,2-b]indol-2-one}<smiles></smiles>

6b

White solid (54.4 mg, 60\%), mp: 211.8-212.5 ${ }^{\circ} \mathrm{C} .{ }^{1} \mathrm{H}$ NMR (500 MHz, $\left.\mathrm{CDCl}_{3}\right) \delta 8.74-8.69(\mathrm{~m}, 1 \mathrm{H}), 7.38-7.29$ (m, 3H), $3.81(\mathrm{~s}, 3 \mathrm{H}), 3.23(\mathrm{dt}, J=15.7,3.8 \mathrm{~Hz}, 1 \mathrm{H}), 3.10-2.99(\mathrm{~m}, 1 \mathrm{H}), 2.49$ (dd, $J=15.6,9.0 \mathrm{~Hz}, 1 \mathrm{H}), 1.97$ (dd, $J=15.6,10.5 \mathrm{~Hz}, 1 \mathrm{H}), 1.94-1.86(\mathrm{~m}, 1 \mathrm{H}), 1.85-1.76(\mathrm{~m}, 1 \mathrm{H}), 1.74(\mathrm{~s}, 3 \mathrm{H}), 1.68-1.49(\mathrm{~m}, 2 \mathrm{H}) .{ }^{13} \mathrm{C} \mathrm{NMR}(125$ $\left.\mathrm{MHz}, \mathrm{CDCl}_{3}\right) \delta 191.63,167.36,147.71,137.49,127.16,123.91,123.88,123.57,109.82,107.05,92.25,36.05$, 30.45, 27.13, 26.90, 23.22, 22.90. HRMS (ESI): calcd. for $\mathrm{C}_{17} \mathrm{H}_{18} \mathrm{~N}_{2} \mathrm{O}_{2}\left([\mathrm{M}+\mathrm{H}]^{+}\right)$: 283.1441, found 283.1449.

\section{3a,12-Dimethyl-3a,4,5,6-tetrahydro-2H-oxazolo[4',5':3,4]azepino[1,2-a]indol-2-one}<smiles>Cc1c2n(c3ccccc13)C1=NC(=O)OC1(C)C2C</smiles>

$8 \mathbf{a}$

White solid (52.6 mg, 98\%), mp: 245.6-246.3 ${ }^{\circ} \mathrm{C} .{ }^{1} \mathrm{H}$ NMR (500 MHz, CDCl3) $\delta 7.70(\mathrm{~d}, J=8.0 \mathrm{~Hz}, 1 \mathrm{H}), 7.43(\mathrm{t}, J$ $=7.6 \mathrm{~Hz}, 1 \mathrm{H}), 7.37(\mathrm{~d}, J=8.4 \mathrm{~Hz}, 1 \mathrm{H}), 7.20(\mathrm{t}, J=7.4 \mathrm{~Hz}, 1 \mathrm{H}), 4.64-4.56(\mathrm{~m}, 1 \mathrm{H}), 3.75-3.66(\mathrm{~m}, 1 \mathrm{H}), 2.65(\mathrm{~s}, 3 \mathrm{H})$, 2.51-2.44 (m, 1H), 2.31-2.18 (m, 2H), 2.08-2.00 (m, 1H), $1.47(\mathrm{~s}, 3 \mathrm{H}) .{ }^{13} \mathrm{C}$ NMR $\left(125 \mathrm{MHz}, \mathrm{CDCl}_{3}\right) \delta 190.94$, $166.19,141.92$, 128.53, 128.12, 127.68, 127.22, 121.80, 121.09, 110.83, 91.02, 47.17, 36.34, 25.59, 23.71, 11.01. HRMS (ESI): calcd. for $\mathrm{C}_{16} \mathrm{H}_{16} \mathrm{~N}_{2} \mathrm{O}_{2}\left([\mathrm{M}+\mathrm{H}]^{+}\right)$: 269.1285, found 269.1288 .

\section{3a,13-Dimethyl-4,5,6,7-tetrahydrooxazolo[4',5' :3,4] azocino[1,2-a]indol-2(3aH)-one}

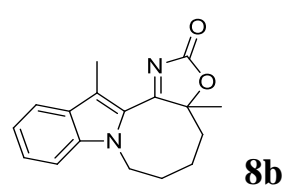

White solid (40.2 mg, 71\%), mp: 123.2-133.4 ${ }^{\circ} \mathrm{C} .{ }^{1} \mathrm{H}$ NMR (500 MHz, $\left.\mathrm{CDCl}_{3}\right) \delta 7.65(\mathrm{~d}, J=7.9 \mathrm{~Hz}, 1 \mathrm{H}), 7.39-$ $7.30(\mathrm{~m}, 2 \mathrm{H}), 7.20(\mathrm{t}, J=7.3 \mathrm{~Hz}, 1 \mathrm{H}), 4.34-4.21(\mathrm{~m}, 1 \mathrm{H}), 4.03-3.93(\mathrm{~m}, 1 \mathrm{H}), 2.40(\mathrm{~s}, 3 \mathrm{H}), 2.35-2.28(\mathrm{~m}, 1 \mathrm{H})$, 2.23-2.13 (m, 1H), 2.09-2.02 (m, 1H), $1.95-1.79(\mathrm{~m}, 2 \mathrm{H}), 1.57-1.47(\mathrm{~m}, 1 \mathrm{H}), 1.40(\mathrm{~s}, 3 \mathrm{H}) .{ }^{13} \mathrm{C}$ NMR $(125 \mathrm{MHz}$, $\left.\mathrm{CDCl}_{3}\right) \delta 194.82,164.97,139.61,128.28,127.14,125.22,120.85,120.51,117.91,109.92,93.86,43.89,33.46$, 27.56, 25.48, 19.01, 9.46. HRMS (ESI): calcd. for $\mathrm{C}_{17} \mathrm{H}_{18} \mathrm{~N}_{2} \mathrm{O}_{2}\left([\mathrm{M}+\mathrm{H}]^{+}\right)$: 283.1441, found 283.1457.

\section{2) General Procedure and Experimental Details for Synthesis of Carbazole Derivatives 10 (Table 2).}

A mixture of substrate $9(0.2 \mathrm{mmol}), \mathrm{Pd}(\mathrm{OAc})_{2}(5 \mathrm{~mol} \%)$ and 2,2'-bipyridine $(6 \mathrm{~mol} \%)$ in HOAc $(0.5 \mathrm{~mL})$ was stirred at $120{ }^{\circ} \mathrm{C}$. Upon completion, the mixture was cooled to room temperature, and then $\mathrm{NaHCO}_{3}$ was added until no $\mathrm{CO}_{2}$ bubbles were generated. The resulting mixture was extracted with DCM three times. The combined 
organic layers were dried over $\mathrm{Na}_{2} \mathrm{SO}_{4}$, filtered, and concentrated under reduced pressure. The residue was purified by flash column chromatography on a silica gel using petroleum ether/EtOAc (10:1 to 3:1) as the eluent to give the desired product $\mathbf{1 0}$.

\section{N-(2,9-Dimethyl-9H-carbazol-1-yl)benzamide}

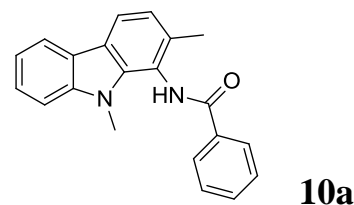

White solid (47.8 mg, 76\%), mp: 191.2-192.6 ${ }^{\circ} \mathrm{C} .{ }^{1} \mathrm{H}$ NMR (500 MHz, $\left.\mathrm{CDCl}_{3}\right) \delta 8.00$ (d, $\left.J=7.6 \mathrm{~Hz}, 1 \mathrm{H}\right), 7.83(\mathrm{~d}, J$ $=7.8 \mathrm{~Hz}, 1 \mathrm{H}), 7.79(\mathrm{~d}, J=7.5 \mathrm{~Hz}, 2 \mathrm{H}), 7.52(\mathrm{dd}, J=15.0,7.5 \mathrm{~Hz}, 2 \mathrm{H}), 7.41(\mathrm{t}, J=7.5 \mathrm{~Hz}, 3 \mathrm{H}), 7.22(\mathrm{t}, J=7.4 \mathrm{~Hz}$, $1 \mathrm{H}), 7.17(\mathrm{~d}, J=8.2 \mathrm{~Hz}, 1 \mathrm{H}), 6.99(\mathrm{~d}, J=7.8 \mathrm{~Hz}, 1 \mathrm{H}), 3.55(\mathrm{~s}, 3 \mathrm{H}), 2.22(\mathrm{~s}, 3 \mathrm{H}) .{ }^{13} \mathrm{C}$ NMR $\left(125 \mathrm{MHz}, \mathrm{CDCl}_{3}\right) \delta$ $167.47,141.72,137.61,134.66,133.94,132.08,128.86,127.40,125.58,123.39,122.67,121.55,119.81,119.53$, $119.19,118.59,108.78,30.59,18.29$. HRMS (ESI): calcd. for $\mathrm{C}_{21} \mathrm{H}_{18} \mathrm{~N}_{2} \mathrm{O}\left([\mathrm{M}+\mathrm{H}]^{+}\right)$: 315.1492 , found 315.1489.

\section{N-(2,9-Dimethyl-9H-carbazol-1-yl)pivalamide}

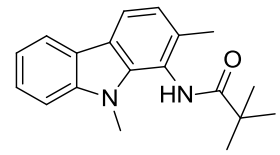

$10 \mathrm{c}$

White solid (44.1 mg, 75\%), mp: 274.5-275.8 ${ }^{\circ} \mathrm{C} .{ }^{1} \mathrm{H}$ NMR (500 MHz, DMSO) $\delta 9.33(\mathrm{~s}, 1 \mathrm{H}), 8.10(\mathrm{~d}, J=7.7 \mathrm{~Hz}$, 1H), $7.98(\mathrm{~d}, J=7.8 \mathrm{~Hz}, 1 \mathrm{H}), 7.53(\mathrm{~d}, J=8.2 \mathrm{~Hz}, 1 \mathrm{H}), 7.43(\mathrm{t}, J=7.5 \mathrm{~Hz}, 1 \mathrm{H}), 7.19(\mathrm{t}, J=7.3 \mathrm{~Hz}, 1 \mathrm{H}), 7.09(\mathrm{~d}, J$ $=7.8 \mathrm{~Hz}, 1 \mathrm{H}), 3.93(\mathrm{~s}, 3 \mathrm{H}), 2.28(\mathrm{~s}, 1 \mathrm{H}), 1.34(\mathrm{~s}, 9 \mathrm{H}) .{ }^{13} \mathrm{C}$ NMR $(125 \mathrm{MHz}, \mathrm{DMSO}) \delta 177.41,141.13,137.51$, 134.37, 125.48, 122.39, 121.91, 121.22, 120.64, 119.74, 118.90, 118.68, 109.02, 38.74, 30.89, 27.46, 17.92. HRMS (ESI): calcd. for $\mathrm{C}_{19} \mathrm{H}_{22} \mathrm{~N}_{2} \mathrm{O}\left([\mathrm{M}+\mathrm{H}]^{+}\right)$: 295.1805, found 295.1809.

\section{N-(2,9-Dimethyl-9H-carbazol-1-yl)-4-methylbenzamide}

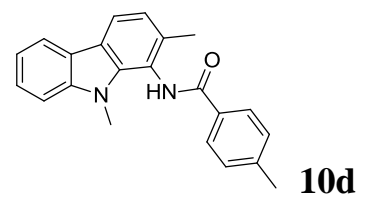

White solid (57.1 mg, 87\%), mp: 240.1-241.6 ${ }^{\circ} \mathrm{C} .{ }^{1} \mathrm{H}$ NMR (500 MHz, DMSO) $\delta 10.16$ (s, 1H), 8.13 (d, $J=7.7 \mathrm{~Hz}$, $1 \mathrm{H}), 8.04-8.00(\mathrm{~m}, 3 \mathrm{H}), 7.53(\mathrm{~d}, J=8.2 \mathrm{~Hz}, 1 \mathrm{H}), 7.44(\mathrm{t}, J=7.6 \mathrm{~Hz}, 1 \mathrm{H}), 7.39(\mathrm{~d}, J=7.7 \mathrm{~Hz}, 2 \mathrm{H}), 7.20(\mathrm{t}, J=7.3$ $\mathrm{Hz}, 1 \mathrm{H}), 7.15(\mathrm{~d}, J=7.8 \mathrm{~Hz}, 1 \mathrm{H}), 3.90(\mathrm{~s}, 3 \mathrm{H}), 2.42(\mathrm{~s}, 3 \mathrm{H}), 2.34(\mathrm{~s}, 3 \mathrm{H}) .{ }^{13} \mathrm{C}$ NMR $(125 \mathrm{MHz}, \mathrm{DMSO}) \delta 166.38$, $141.78,141.20,137.41,134.48,131.29,129.18,127.65,125.52,122.48,121.93,121.25,120.22,119.77,119.52$, 118.99, 118.94, 109.07, 30.61, 21.04, 17.96. HRMS (ESI): calcd. for $\mathrm{C}_{22} \mathrm{H}_{20} \mathrm{~N}_{2} \mathrm{O}\left([\mathrm{M}+\mathrm{H}]^{+}\right)$: 329.1648 found 329.1640.

\section{4-Bromo-N-(2,9-dimethyl-9H-carbazol-1-yl)benzamide}

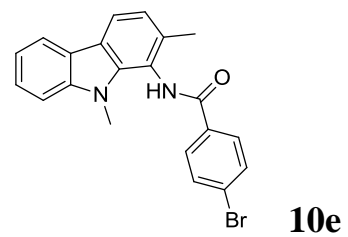

White solid (70.6 mg, 90\%), mp: 264.3-264.9 ${ }^{\circ} \mathrm{C} .{ }^{1} \mathrm{H}$ NMR (500 MHz, DMSO) $\delta 10.35$ (s, 1H), 8.13 (d, $J=7.7 \mathrm{~Hz}$, 
$1 \mathrm{H}), 8.06-8.03(\mathrm{~m}, 3 \mathrm{H}), 7.82(\mathrm{~d}, J=8.4 \mathrm{~Hz}, 2 \mathrm{H}), 7.54(\mathrm{~d}, J=8.2 \mathrm{~Hz}, 1 \mathrm{H}), 7.44(\mathrm{t}, J=7.5 \mathrm{~Hz}, 1 \mathrm{H}), 7.21(\mathrm{t}, J=7.3$ $\mathrm{Hz}, 1 \mathrm{H}), 7.16(\mathrm{~d}, J=7.9 \mathrm{~Hz}, 1 \mathrm{H}), 3.90(\mathrm{~s}, 1 \mathrm{H}), 2.35$ (s, 1H). ${ }^{13} \mathrm{C}$ NMR $(125 \mathrm{MHz}, \mathrm{DMSO}) \delta 165.64,141.22$, 137.28, 134.39, 133.19, 131.77, 129.77, 125.70, 125.61, 122.57, 121.91, 121.31, 119.83, 119.15, 119.08, 109.15, 30.67, 17.98. HRMS (ESI): calcd. for $\mathrm{C}_{21} \mathrm{H}_{17} \mathrm{BrN}_{2} \mathrm{O}\left([\mathrm{M}+\mathrm{H}]^{+}\right)$: 393.0597, found 393.0596.

\section{N-(2,9-Dimethyl-9H-carbazol-1-yl)-4-nitrobenzamide}

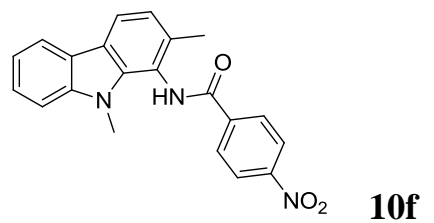

White solid (61.1 mg, 85\%), mp: 257.8-258.6 ${ }^{\circ} \mathrm{C} .{ }^{1} \mathrm{H}$ NMR (500 MHz, DMSO) $\delta 10.63$ (s, $\left.1 \mathrm{H}\right), 8.44(\mathrm{~d}, J=8.2 \mathrm{~Hz}$, 2H), $8.33(\mathrm{~d}, J=8.2 \mathrm{~Hz}, 2 \mathrm{H}), 8.14(\mathrm{~d}, J=7.5 \mathrm{~Hz}, 1 \mathrm{H}), 8.07(\mathrm{~d}, J=7.7 \mathrm{~Hz}, 1 \mathrm{H}), 7.55(\mathrm{~d}, J=8.0 \mathrm{~Hz}, 1 \mathrm{H}), 7.45(\mathrm{t}, J$ $=7.3 \mathrm{~Hz}, 1 \mathrm{H}), 7.28-7.13(\mathrm{~m}, 2 \mathrm{H}), 3.93(\mathrm{~s}, 3 \mathrm{H}), 2.37(\mathrm{~s}, 3 \mathrm{H}) .{ }^{13} \mathrm{C}$ NMR $(125 \mathrm{MHz}, \mathrm{DMSO}) \delta 165.01,149.43$, $141.23,139.70,137.14,134.29,129.16,125.66,123.93,122.66,121.88,121.35,119.85,119.46,119.31,119.13$, 109.17, 30.71, 17.97. HRMS (ESI): calcd. for $\mathrm{C}_{21} \mathrm{H}_{17} \mathrm{~N}_{3} \mathrm{O}_{3}\left([\mathrm{M}+\mathrm{H}]^{+}\right)$: 360.1343 , found 360.1346.

\section{N-(2-Ethyl-9-methyl-9H-carbazol-1-yl)benzamide}

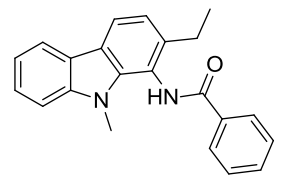

\section{$10 \mathrm{~g}$}

White solid (55.8 mg, 85\%), mp: 163.2-164.6 ${ }^{\circ} \mathrm{C} .{ }^{1} \mathrm{H}$ NMR (500 MHz, $\left.\mathrm{CDCl}_{3}\right) \delta 8.00(\mathrm{~d}, J=7.6 \mathrm{~Hz}, 1 \mathrm{H}), 7.88(\mathrm{~d}, J$ $=7.9 \mathrm{~Hz}, 1 \mathrm{H}), 7.79(\mathrm{~d}, J=7.4 \mathrm{~Hz}, 2 \mathrm{H}), 7.64(\mathrm{~s}, 1 \mathrm{H}), 7.51(\mathrm{t}, J=7.4 \mathrm{~Hz}, 1 \mathrm{H}), 7.44-7.35(\mathrm{~m}, 3 \mathrm{H}), 7.21(\mathrm{t}, J=7.4$ $\mathrm{Hz}, 1 \mathrm{H}), 7.15(\mathrm{~d}, J=8.2 \mathrm{~Hz}, 1 \mathrm{H}), 7.03(\mathrm{~d}, J=7.9 \mathrm{~Hz}, 1 \mathrm{H}), 3.55(\mathrm{~s}, 3 \mathrm{H}), 2.60$ (d, $J=7.0 \mathrm{~Hz}, 2 \mathrm{H}), 1.16(\mathrm{t}, J=7.6$ $\mathrm{Hz}, 3 \mathrm{H}) .{ }^{13} \mathrm{C}$ NMR $\left(125 \mathrm{MHz}, \mathrm{CDCl}_{3}\right) \delta 167.90,141.81,140.30,137.68,133.99,132.08,128.89,127.40,125.54$, 123.36, 122.67, 119.87, 119.83, 119.76, 119.14, 117.99, 108.73, 30.55, 24.89, 15.13. HRMS (ESI): calcd. for $\mathrm{C}_{22} \mathrm{H}_{20} \mathrm{~N}_{2} \mathrm{O}\left([\mathrm{M}+\mathrm{H}]^{+}\right): 329.1648$, found 329.1644 .

\section{N-(9-Methyl-2-phenyl-9H-carbazol-1-yl)-4-nitrobenzamide}

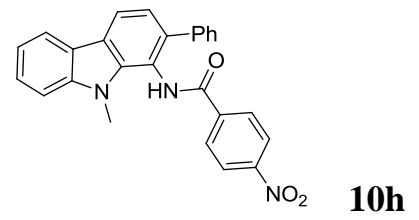

White solid (65.7 mg, 78\%), mp: 268.4-269.2 ${ }^{\circ} \mathrm{C} .{ }^{1} \mathrm{H}$ NMR (500 MHz, DMSO) $\delta 10.58(\mathrm{~s}, 1 \mathrm{H}), 8.35$ (d, $J=8.6 \mathrm{~Hz}$, $2 \mathrm{H}), 8.24(\mathrm{dd}, J=13.5,7.9 \mathrm{~Hz}, 2 \mathrm{H}), 8.04(\mathrm{~d}, J=8.6 \mathrm{~Hz}, 2 \mathrm{H}), 7.61(\mathrm{~d}, J=8.2 \mathrm{~Hz}, 1 \mathrm{H}), 7.54-7.46(\mathrm{~m}, 3 \mathrm{H}), 7.39(\mathrm{t}$, $J=7.5 \mathrm{~Hz}, 2 \mathrm{H}), 7.34-7.22$ (m, 3H), 3.95 (s, 3H). ${ }^{13} \mathrm{C}$ NMR (125MHz, DMSO) $\delta 165.90,149.30,141.64,139.80$, $139.75,139.05,137.22$, 129.06, 128.88, 128.00, 127.04, 126.23, 123.83, 121.62, 121.34, 120.21, 119.82, 119.41, 118.15, 109.34, 30.78. HRMS (ESI): calcd. for $\mathrm{C}_{26} \mathrm{H}_{19} \mathrm{~N}_{3} \mathrm{O}_{3}\left([\mathrm{M}+\mathrm{H}]^{+}\right)$: 422.1499 , found 422.1498 .

\section{3,9-Dimethyl-9H-carbazol-4-amine}

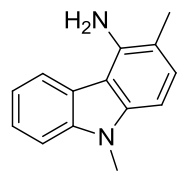


White solid (31.5 mg, 75\%), mp: 175.4-176.9 ${ }^{\circ} \mathrm{C} .{ }^{1} \mathrm{H}$ NMR $\left(500 \mathrm{MHz}, \mathrm{CDCl}_{3}\right) \delta 7.98(\mathrm{~d}, J=7.8 \mathrm{~Hz}, 1 \mathrm{H}), 7.42(\mathrm{t}, J$ $=7.6 \mathrm{~Hz}, 1 \mathrm{H}), 7.36(\mathrm{~d}, J=8.0 \mathrm{~Hz}, 1 \mathrm{H}), 7.24-7.18(\mathrm{~m}, 2 \mathrm{H}), 6.78(\mathrm{~d}, J=8.1 \mathrm{~Hz}, 1 \mathrm{H}), 4.32(\mathrm{brs}, 2 \mathrm{H}), 3.77(\mathrm{~s}, 1 \mathrm{H})$, $2.34(\mathrm{~s}, 3 \mathrm{H}) .{ }^{13} \mathrm{C}$ NMR $\left(125 \mathrm{MHz}, \mathrm{CDCl}_{3}\right) \delta 141.37,140.79,140.43,128.59,124.23,122.44,120.80,118.67$, 111.91, 109.96, 108.09, 98.53, 29.20, 16.83. HRMS (ESI): calcd. for $\mathrm{C}_{14} \mathrm{H}_{14} \mathrm{~N}_{2}\left([\mathrm{M}+\mathrm{H}]^{+}\right)$: 211.1230, found 211.1236.

\section{3-Ethyl-9-methyl-9H-carbazol-4-amine}

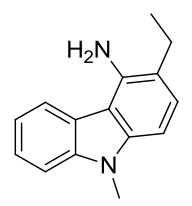

$10 \mathrm{j}$

White solid (53.2 mg, 81\%), mp: 170.7-171.9 ${ }^{\circ} \mathrm{C} .{ }^{1} \mathrm{H}$ NMR $\left(500 \mathrm{MHz}, \mathrm{CDCl}_{3}\right) \delta 7.97(\mathrm{~d}, J=7.7 \mathrm{~Hz}, 1 \mathrm{H}), 7.41(\mathrm{t}, J=7.5$ $\mathrm{Hz}, 1 \mathrm{H}), 7.34(\mathrm{~d}, J=8.0 \mathrm{~Hz}, 1 \mathrm{H}), 7.27-7.16(\mathrm{~m}, 2 \mathrm{H}), 6.80(\mathrm{~d}, J=8.1 \mathrm{~Hz}, 1 \mathrm{H}), 4.29$ (brs, 2H), 3.74 (s, 3H), 2.71-2.66 $(\mathrm{m}, 2 \mathrm{H}), 1.31(\mathrm{t}, J=7.5 \mathrm{~Hz}, 3 \mathrm{H}) .{ }^{13} \mathrm{C} \mathrm{NMR}\left(125 \mathrm{MHz}, \mathrm{CDCl}_{3}\right) \delta 141.20,140.83,139.92,126.96,124.22,122.57,120.84$, 118.67, 118.22, 110.25, 108.07, 98.78, 29.14, 23.83, 14.33. HRMS (ESI): calcd. for $\mathrm{C}_{15} \mathrm{H}_{16} \mathrm{~N}_{2}\left([\mathrm{M}+\mathrm{H}]^{+}\right): 225.1386$, found 225.1388 .

Compounds 13 and 14 were prepared according to the procedures for the synthesis of compound 2 and 10 (Scheme 3).

3a,5,10-Trimethyl-4,5-dihydro-3aH-oxazolo[4,5-a]carbazol-2(10H)-one

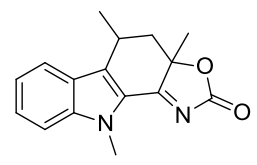

13

White solid (47 mg, 88\%, $d r=1: 1$, unseparated diastereoisomers). The data of diastereoisomers: ${ }^{1} \mathrm{H}$ NMR (500 $\left.\mathrm{MHz}, \mathrm{CDCl}_{3}\right) \delta 7.84(\mathrm{~d}, J=8.3 \mathrm{~Hz}, 1 \mathrm{H}), 7.68(\mathrm{~d}, J=8.1 \mathrm{~Hz}, 1 \mathrm{H}), 7.49-7.44(\mathrm{~m}, 2 \mathrm{H}), 7.43-7.39(\mathrm{~m}, 2 \mathrm{H}), 7.22-$ $7.15(\mathrm{~m}, 2 \mathrm{H}), 4.10(\mathrm{~s}, 3 \mathrm{H}), 4.09(\mathrm{~s}, 3 \mathrm{H}), 3.75-3.66(\mathrm{~m}, 1 \mathrm{H}), 3.55-3.45(\mathrm{~m}, 1 \mathrm{H}), 2.56(\mathrm{dd}, J=12.5,5.0 \mathrm{~Hz}, 1 \mathrm{H})$, $2.50(\mathrm{dd}, J=13.2,8.0 \mathrm{~Hz}, 1 \mathrm{H}), 2.32(\mathrm{~d}, J=13.3 \mathrm{~Hz}, 1 \mathrm{H}), 1.88(\mathrm{t}, J=11.6 \mathrm{~Hz}, 1 \mathrm{H}), 1.71(\mathrm{~s}, 3 \mathrm{H}), 1.68(\mathrm{~d}, J=7.6$ $\mathrm{Hz}, 3 \mathrm{H}), 1.61(\mathrm{~d}, J=6.8 \mathrm{~Hz}, 3 \mathrm{H}), 1.57(\mathrm{~s}, 3 \mathrm{H}) .{ }^{13} \mathrm{C} \mathrm{NMR}\left(125 \mathrm{MHz}, \mathrm{CDCl}_{3}\right) \delta 188.78,188.67,166.90,166.83$, $142.38,142.33,131.70,127.88,127.61,125.37,125.22$, 124.20, 124.05, 122.68, 121.65, 121.01, 120.88, 120.08, 110.96, 110.88, 87.24, 87.23, 44.30, 41.69, 31.46, 28.98, 27.99, 26.69, 23.72, 21.87, 21.38. HRMS (ESI): calcd. for $\mathrm{C}_{16} \mathrm{H}_{16} \mathrm{~N}_{2} \mathrm{O}_{2}\left([\mathrm{M}+\mathrm{H}]^{+}\right): 269.1285$ found 269.1282.

\section{N-(2,4,9-Trimethyl-9H-carbazol-1-yl)benzamide}

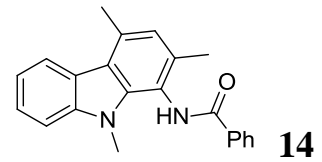

White solid (53 mg, 81\%), mp: 197.6-198.3 ${ }^{\circ} \mathrm{C} .{ }^{1} \mathrm{H}$ NMR (500 MHz, DMSO) $\delta 10.23$ (s, 1H), $8.18-8.06$ (m, 3H), 7.66-7.55 (m, 4H), $7.46(\mathrm{t}, J=7.6 \mathrm{~Hz}, 1 \mathrm{H}), 7.24(\mathrm{t}, J=7.4 \mathrm{~Hz}, 1 \mathrm{H}), 6.97(\mathrm{~s}, 1 \mathrm{H}), 3.94(\mathrm{~s}, 3 \mathrm{H}), 2.81(\mathrm{~s}, 3 \mathrm{H}), 2.33$ (s, $3 \mathrm{H}) .{ }^{13} \mathrm{C}$ NMR (125 MHz, DMSO) $\delta 166.55,141.20,137.32,134.19,134.05,131.73,131.23,128.66,127.63$, $124.91,122.96,122.48,121.72,120.66,119.08,117.90,108.86,30.66,20.32,17.80$. HRMS (ESI): calcd. for $\mathrm{C}_{22} \mathrm{H}_{20} \mathrm{~N}_{2} \mathrm{O}\left([\mathrm{M}+\mathrm{H}]^{+}\right): 329.1648$ found 329.1647 .

\section{3) Procedure for Synthesis of Compound (Scheme 4).}

To a solution of compound $\mathbf{2 y}(0.2 \mathrm{mmol})$ in DMSO $(2 \mathrm{~mL})$, L-proline $(20 \mathrm{~mol} \%)$ was added. The mixture 
was stirred at $\mathrm{rt}$ for $2 \mathrm{~h}$. Upon completion, brine was added. The resulting mixture was extracted with DCM three times. The combined organic layers were dried over $\mathrm{Na}_{2} \mathrm{SO}_{4}$, filtered, and concentrated under reduced pressure. The residue was purified by flash column chromatography on a silica gel using petroleum ether/EtOAc (10:1 to 5:1) as the eluent to give the desired product $\mathbf{1 5}$.

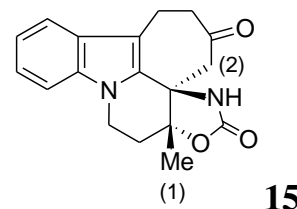

White solid ( $61.4 \mathrm{mg}, 99 \%$ ), mp: 268.2-269.3 ${ }^{\circ} \mathrm{C} .{ }^{1} \mathrm{H}$ NMR (500 MHz, DMSO) $\delta 8.36$ (brs, $1 \mathrm{H}$ ), 7.59 (d, $J=7.9$ $\mathrm{Hz}, 1 \mathrm{H}), 7.45(\mathrm{~d}, J=8.2 \mathrm{~Hz}, 1 \mathrm{H}), 7.20(\mathrm{t}, J=7.4 \mathrm{~Hz}, 1 \mathrm{H}), 7.08(\mathrm{t}, J=7.4 \mathrm{~Hz}, 1 \mathrm{H}), 4.19-4.09(\mathrm{~m}, 2 \mathrm{H}), 3.29(\mathrm{~d}, J$ $=12.4 \mathrm{~Hz}, 1 \mathrm{H}), 3.14-3.01(\mathrm{~m}, 2 \mathrm{H}), 2.85(\mathrm{~d}, J=12.4 \mathrm{~Hz}, 1 \mathrm{H}), 2.82-2.75(\mathrm{~m}, 1 \mathrm{H}), 2.70-2.61(\mathrm{~m}, 1 \mathrm{H}), 2.38-$ $2.31(\mathrm{~m}, 1 \mathrm{H}), 2.09-2.02(\mathrm{~m}, 1 \mathrm{H}), 1.50(\mathrm{~s}, 3 \mathrm{H}) .{ }^{13} \mathrm{C}$ NMR (125 MHz, DMSO) $\delta 208.72,155.93,134.56,131.28$, 126.72, 122.07, 119.25, 118.42, 113.00, 109.61, 82.70, 58.37, 50.23, 43.31, 37.73, 34.76, 20.44, 17.88. HRMS (ESI): calcd. for $\mathrm{C}_{18} \mathrm{H}_{18} \mathrm{~N}_{2} \mathrm{O}_{3}\left([\mathrm{M}+\mathrm{H}]^{+}\right): 311.1390$, found 311.1392 .

Irradiation (transient NOE) at $\delta=1.50 \mathrm{ppm}(\mathrm{H}-1)$ enhances the signals at $\delta=3.29 \mathrm{ppm}(\mathrm{H}-2,0.60 \%), \delta=2.85 \mathrm{ppm}$ $(\mathrm{H}-2,0.87 \%)$.

\section{Proposed Mechanism}

Proposed mechanism was illustrated in Scheme S1 for this Pd-catalyzed cyclization with the exemplification of C-3 substituted indole substrate. In analogy to other processes involving Pd-catalyzed C-H bond functionalization, this catalytic process may involve the following key steps: (1) a direct palladation at the C-3 position of an indole core with [(bpy) $\left.\mathrm{Pd}(\mathrm{OAc})_{2}\right] \mathbf{A}$ gives a palladium complex $\mathbf{B}$, which undergoes a 1,2-migration of palla dium to give the species $\mathbf{C}$; (2) the species $\mathbf{C}$ undergoes the loss of HOAc with generation of 2-indolyl-palladium complex $\mathbf{D}$; (3) the addition of the complex $\mathbf{D}$ across the cyano group in close prox imity to the palladium center, affords an iminopalladium species $\mathbf{E},{ }^{9}$ which reacts with O-ethoxycarbonyl group giving the species $\mathbf{F}$ which would furnish product 2 and regenerating $\mathrm{Pd}(\mathrm{II})$ species. The cyclizations of $\mathrm{N}-1$ substituted indole analogues follow the similar reaction pathway, while C-2 substituted indole analogues may involve a 3-indolyl-palladium complex. Additionally, with respect to the cyclization of substrate $\mathbf{9}$, the reaction between iminopalladium species $\mathbf{E}$ and neighboring O-arylacyl group would led to amide species $\mathbf{G}$. The protonation of hydroxyl group of $\mathbf{G}$ followed by the elimination gives $\mathbf{H}$, which undergoes an aromatization to provide carbazolamine $\mathbf{9}$.

\section{Scheme S1. Proposed Mechanism}

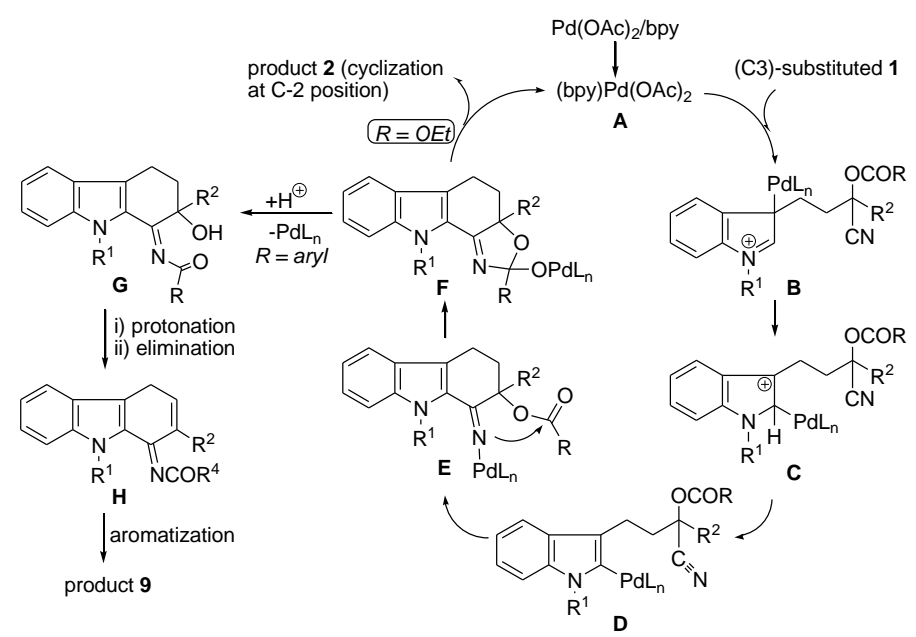




\section{References}

1. N. Azizi, F. Arynasab, M. R. Saidi Org. Biomol. Chem., 2006, 4, 4275.

2. A. Baeza, C. Nájera, M. G. Retamosa, J. M. Sansano Synthesis 2005, 16, 2787.

3. L. Fra, A. Millán, J. A. Souto, K. Muñiz Angew. Chem. Int. Ed., 2014, 53, 7349.

4. G. Zhan, Q. He, X. Yuan, Y.- C. Chen, Org. Lett. 2014, 16, 6000.

5. H. Kuday, F. Sonmez, C. Bilen, E. Yavuz, N. Cencer, M. Kucikislamoglu MioMed Res. Int. 2014, 2014, $594879 / 1$.

6. C.-E. Yeom, M. J. Kim, B. M, Kim Tetrahedron 2007, 63, 904.

7. B. Zhou, Z. Luo, Y. Li Chem. Eur. J. 2013, 19, 4428.

8. N. P. Grimster, C. Gauntlett, C. R. A. Godfrey, M. J. Gaunt, Angew. Chem. Int. Ed. 2005, 44, 3125.

9. The possibility that intermediate $\mathbf{E}$ would undergo protonation to generate imine which subsequently reacts with the tethered OCOR group to give corresponding intermediate also cannot be ruled out. 
VI. Crystal Data and Structure Refinement

1) Compound $\mathbf{2 a}$
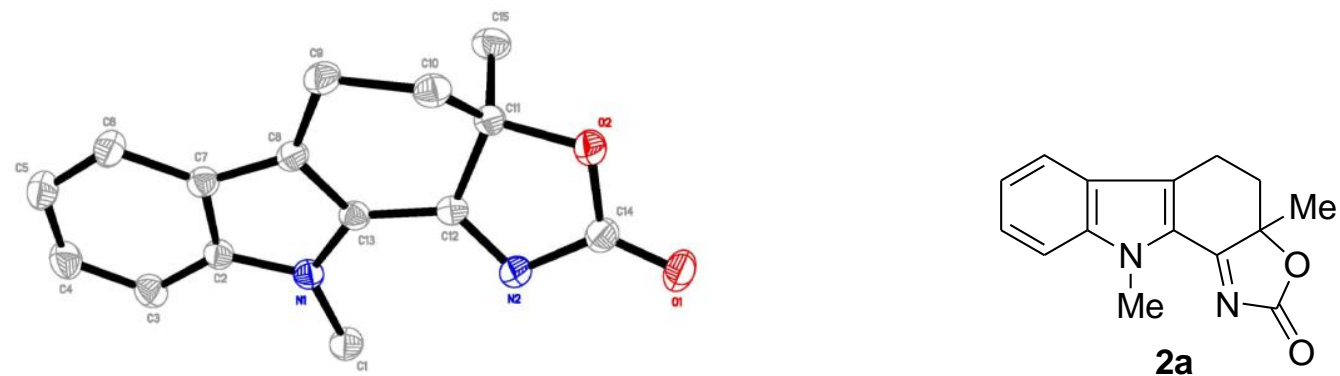

CCDC 1488848

Table S2. Crystal data and structure refinement for $\mathbf{2 a}$.

Identification code

Empirical formula

Formula weight

Temperature

Wavelength

Crystal system, space group

Unit cell dimensions

Volume

Z, Calculated density

Absorption coefficient

$\mathrm{F}(000)$

Crystal size

Theta range for data collection $\quad 3.37$ to $27.48 \mathrm{deg}$

Limiting indices 2a

C15 H14 N2 O2

254.28

293(2) K

$0.71073 \mathrm{~A}$

Monoclinic, P 21/n

$$
\begin{aligned}
& \mathrm{a}=8.5448(17) \text { A } \quad \text { alpha }=90 \mathrm{deg} . \\
& \mathrm{b}=17.593(4) \mathrm{A} \quad \text { beta }=115.74(3) \mathrm{deg} \text {. } \\
& \mathrm{c}=9.2448(18) \text { A } \quad \text { gamma }=90 \mathrm{deg} .
\end{aligned}
$$

$1251.8(4) \mathrm{A}^{\wedge} 3$

4, $\quad 1.349 \mathrm{Mg} / \mathrm{m}^{\wedge} 3$

$0.091 \mathrm{~mm}^{\wedge}-1$

536

$0.13 \times 0.12 \times 0.10 \mathrm{~mm}$

$-11<=\mathrm{h}<=11,-22<=\mathrm{k}<=22,-11<=1<=11$ 
Reflections collected / unique $\quad 11838 / 2833[\mathrm{R}($ int $)=0.0389]$

Completeness to theta $=27.48 \quad 98.9 \%$

Absorption correction Semi-empirical from equivalents

Max. and min. transmission $\quad 0.9909$ and 0.9882

Refinement method $\quad$ Full-matrix least-squares on $\mathrm{F}^{\wedge} 2$

Data / restraints / parameters 2833 / 0 / 173

Goodness-of-fit on $\mathrm{F}^{\wedge} 2$

Final $\mathrm{R}$ indices $[\mathrm{I}>2 \operatorname{sigma}(\mathrm{I})] \quad \mathrm{R} 1=0.0458, \mathrm{wR} 2=0.1260$

$\mathrm{R}$ indices (all data) $\quad \mathrm{R} 1=0.0637, \mathrm{wR} 2=0.1423$

Largest diff. peak and hole $\quad 0.301$ and -0.210 e. $\mathrm{A}^{\wedge}-3$

Table S3. Atomic coordinates ( x 10^4) and equivalent isotropic displacement parameters $\left(\mathrm{A}^{\wedge} 2 \times 10^{\wedge} 3\right)$ for $\mathrm{ZYJ}$.

$\mathrm{U}(\mathrm{eq})$ is defined as one third of the trace of the orthogonalized

Uij tensor.

\begin{tabular}{lrrrr}
\hline & & & & \\
& $\mathrm{X}$ & $\mathrm{Z}$ & $\mathrm{U}(\mathrm{eq})$ \\
\hline & & & & \\
$\mathrm{C}(1)$ & $7494(2)$ & $1741(1)$ & $2132(2)$ & $45(1)$ \\
$\mathrm{C}(2)$ & $10703(2)$ & $1526(1)$ & $3064(2)$ & $35(1)$ \\
$\mathrm{C}(3)$ & $11323(2)$ & $2269(1)$ & $3241(2)$ & $45(1)$ \\
$\mathrm{C}(4)$ & $13090(3)$ & $2364(1)$ & $3846(2)$ & $53(1)$ \\
$\mathrm{C}(5)$ & $14235(2)$ & $1746(1)$ & $4258(2)$ & $53(1)$ \\
$\mathrm{C}(6)$ & $13637(2)$ & $1017(1)$ & $4072(2)$ & $46(1)$ \\
$\mathrm{C}(7)$ & $11829(2)$ & $890(1)$ & $3463(2)$ & $35(1)$ \\
$\mathrm{C}(8)$ & $10780(2)$ & $229(1)$ & $3120(2)$ & $35(1)$ \\
$\mathrm{C}(9)$ & $11274(2)$ & $-596(1)$ & $3367(2)$ & $41(1)$ \\
$\mathrm{C}(10)$ & $9763(2)$ & $-1104(1)$ & $3241(2)$ & $39(1)$ \\
$\mathrm{C}(11)$ & $8082(2)$ & $-848(1)$ & $1845(2)$ & $35(1)$ \\
$\mathrm{C}(12)$ & $7689(2)$ & $-45(1)$ & $2114(2)$ & $32(1)$ \\
$\mathrm{C}(13)$ & $9080(2)$ & $484(1)$ & $2533(2)$ & $33(1)$ \\
& & & &
\end{tabular}




$\begin{array}{lrrrr}\mathrm{C}(14) & 5406(2) & -685(1) & 1782(2) & 41(1) \\ \mathrm{C}(15) & 8078(2) & -962(1) & 209(2) & 47(1) \\ \mathrm{N}(1) & 9023(2) & 1270(1) & 2522(1) & 36(1) \\ \mathrm{N}(2) & 6137(2) & 49(1) & 2005(2) & 39(1) \\ \mathrm{O}(1) & 3965(2) & -842(1) & 1590(2) & 56(1) \\ \mathrm{O}(2) & 6566(2) & -1230(1) & 1822(1) & 43(1)\end{array}$

Table S4. Bond lengths [A] and angles [deg] for ZYJ.

$\begin{array}{ll}\mathrm{C}(1)-\mathrm{N}(1) & 1.454(2) \\ \mathrm{C}(1)-\mathrm{H}(1 \mathrm{~A}) & 0.9600 \\ \mathrm{C}(1)-\mathrm{H}(1 \mathrm{~B}) & 0.9600 \\ \mathrm{C}(1)-\mathrm{H}(1 \mathrm{C}) & 0.9600 \\ \mathrm{C}(2)-\mathrm{N}(1) & 1.374(2) \\ \mathrm{C}(2)-\mathrm{C}(3) & 1.393(2) \\ \mathrm{C}(2)-\mathrm{C}(7) & 1.416(2) \\ \mathrm{C}(3)-\mathrm{C}(4) & 1.374(3) \\ \mathrm{C}(3)-\mathrm{H}(3) & 0.9300 \\ \mathrm{C}(4)-\mathrm{C}(5) & 1.400(3) \\ \mathrm{C}(4)-\mathrm{H}(4) & 0.9300 \\ \mathrm{C}(5)-\mathrm{C}(6) & 1.364(3) \\ \mathrm{C}(5)-\mathrm{H}(5) & 0.9300 \\ \mathrm{C}(6)-\mathrm{C}(7) & 1.413(2) \\ \mathrm{C}(6)-\mathrm{H}(6) & 0.9300 \\ \mathrm{C}(7)-\mathrm{C}(8) & 1.418(2) \\ \mathrm{C}(8)-\mathrm{C}(13) & 1.386(2) \\ \mathrm{C}(8)-\mathrm{C}(9) & 1.501(2) \\ \mathrm{C}(9)-\mathrm{C}(10) & 1.532(2) \\ \mathrm{C}(9)-\mathrm{H}(9 \mathrm{~A}) & 0.9700 \\ \mathrm{C}(9)-\mathrm{H}(9 \mathrm{~B}) & 0.9700 \\ \mathrm{C}(10)-\mathrm{C}(11) & 1.524(2) \\ \mathrm{C}(10)-\mathrm{H}(10 \mathrm{~A}) & 0.9700 \\ \mathrm{C}(10)-\mathrm{H}(10 \mathrm{~B}) & 0.9700 \\ \mathrm{C}(11)-\mathrm{O}(2) & 1.4512(18) \\ \mathrm{C}(11)-\mathrm{C}(12) & 1.498(2) \\ \mathrm{C}(11)-\mathrm{C}(15) & 1.525(2) \\ \mathrm{C}(12)-\mathrm{N}(2) & 1.296(2) \\ \mathrm{C}(12)-\mathrm{C}(13) & 1.424(2) \\ \mathrm{C}(13)-\mathrm{N}(1) & 1.384(2) \\ \mathrm{C}(14)-\mathrm{O}(1) & 1.197(2) \\ \mathrm{C}(14)-\mathrm{O}(2) & 1.368(2) \\ & \\ & \\ & \end{array}$




\begin{tabular}{|c|c|}
\hline $\mathrm{C}(14)-\mathrm{N}(2)$ & $1.410(2)$ \\
\hline $\mathrm{C}(15)-\mathrm{H}(15 \mathrm{~A})$ & 0.9600 \\
\hline C(15)-H(15B) & 0.9600 \\
\hline $\mathrm{C}(15)-\mathrm{H}(15 \mathrm{C})$ & 0.9600 \\
\hline $\mathrm{N}(1)-\mathrm{C}(1)-\mathrm{H}(1 \mathrm{~A})$ & 109.5 \\
\hline $\mathrm{N}(1)-\mathrm{C}(1)-\mathrm{H}(1 \mathrm{~B})$ & 109.5 \\
\hline $\mathrm{H}(1 \mathrm{~A})-\mathrm{C}(1)-\mathrm{H}(1 \mathrm{~B})$ & 109.5 \\
\hline $\mathrm{N}(1)-\mathrm{C}(1)-\mathrm{H}(1 \mathrm{C})$ & 109.5 \\
\hline $\mathrm{H}(1 \mathrm{~A})-\mathrm{C}(1)-\mathrm{H}(1 \mathrm{C})$ & 109.5 \\
\hline $\mathrm{H}(1 \mathrm{~B})-\mathrm{C}(1)-\mathrm{H}(1 \mathrm{C})$ & 109.5 \\
\hline $\mathrm{N}(1)-\mathrm{C}(2)-\mathrm{C}(3)$ & $129.31(15)$ \\
\hline $\mathrm{N}(1)-\mathrm{C}(2)-\mathrm{C}(7)$ & $108.72(14)$ \\
\hline $\mathrm{C}(3)-\mathrm{C}(2)-\mathrm{C}(7)$ & $121.97(15)$ \\
\hline $\mathrm{C}(4)-\mathrm{C}(3)-\mathrm{C}(2)$ & $117.20(17)$ \\
\hline $\mathrm{C}(4)-\mathrm{C}(3)-\mathrm{H}(3)$ & 121.4 \\
\hline $\mathrm{C}(2)-\mathrm{C}(3)-\mathrm{H}(3)$ & 121.4 \\
\hline$C(3)-C(4)-C(5)$ & $122.05(17)$ \\
\hline $\mathrm{C}(3)-\mathrm{C}(4)-\mathrm{H}(4)$ & 119.0 \\
\hline $\mathrm{C}(5)-\mathrm{C}(4)-\mathrm{H}(4)$ & 119.0 \\
\hline$C(6)-C(5)-C(4)$ & $121.11(17)$ \\
\hline $\mathrm{C}(6)-\mathrm{C}(5)-\mathrm{H}(5)$ & 119.4 \\
\hline $\mathrm{C}(4)-\mathrm{C}(5)-\mathrm{H}(5)$ & 119.4 \\
\hline$C(5)-C(6)-C(7)$ & $118.92(17)$ \\
\hline $\mathrm{C}(5)-\mathrm{C}(6)-\mathrm{H}(6)$ & 120.5 \\
\hline$C(7)-C(6)-H(6)$ & 120.5 \\
\hline$C(6)-C(7)-C(2)$ & $118.74(15)$ \\
\hline $\mathrm{C}(6)-\mathrm{C}(7)-\mathrm{C}(8)$ & $133.94(15)$ \\
\hline $\mathrm{C}(2)-\mathrm{C}(7)-\mathrm{C}(8)$ & $107.32(14)$ \\
\hline $\mathrm{C}(13)-\mathrm{C}(8)-\mathrm{C}(7)$ & $105.95(14)$ \\
\hline $\mathrm{C}(13)-\mathrm{C}(8)-\mathrm{C}(9)$ & $123.39(14)$ \\
\hline $\mathrm{C}(7)-\mathrm{C}(8)-\mathrm{C}(9)$ & $130.61(14)$ \\
\hline $\mathrm{C}(8)-\mathrm{C}(9)-\mathrm{C}(10)$ & $112.37(13)$ \\
\hline $\mathrm{C}(8)-\mathrm{C}(9)-\mathrm{H}(9 \mathrm{~A})$ & 109.1 \\
\hline $\mathrm{C}(10)-\mathrm{C}(9)-\mathrm{H}(9 \mathrm{~A})$ & 109.1 \\
\hline $\mathrm{C}(8)-\mathrm{C}(9)-\mathrm{H}(9 \mathrm{~B})$ & 109.1 \\
\hline $\mathrm{C}(10)-\mathrm{C}(9)-\mathrm{H}(9 \mathrm{~B})$ & 109.1 \\
\hline $\mathrm{H}(9 \mathrm{~A})-\mathrm{C}(9)-\mathrm{H}(9 \mathrm{~B})$ & 107.9 \\
\hline $\mathrm{C}(11)-\mathrm{C}(10)-\mathrm{C}(9)$ & $110.19(13)$ \\
\hline $\mathrm{C}(11)-\mathrm{C}(10)-\mathrm{H}(10 \mathrm{~A})$ & 109.6 \\
\hline $\mathrm{C}(9)-\mathrm{C}(10)-\mathrm{H}(10 \mathrm{~A})$ & 109.6 \\
\hline $\mathrm{C}(11)-\mathrm{C}(10)-\mathrm{H}(10 \mathrm{~B})$ & 109.6 \\
\hline $\mathrm{C}(9)-\mathrm{C}(10)-\mathrm{H}(10 \mathrm{~B})$ & 109.6 \\
\hline $\mathrm{H}(10 \mathrm{~A})-\mathrm{C}(10)-\mathrm{H}(10 \mathrm{~B})$ & 108.1 \\
\hline
\end{tabular}




$\begin{array}{lc}\mathrm{O}(2)-\mathrm{C}(11)-\mathrm{C}(12) & 100.17(11) \\ \mathrm{O}(2)-\mathrm{C}(11)-\mathrm{C}(10) & 112.51(13) \\ \mathrm{C}(12)-\mathrm{C}(11)-\mathrm{C}(10) & 109.60(13) \\ \mathrm{O}(2)-\mathrm{C}(11)-\mathrm{C}(15) & 107.93(13) \\ \mathrm{C}(12)-\mathrm{C}(11)-\mathrm{C}(15) & 112.57(13) \\ \mathrm{C}(10)-\mathrm{C}(11)-\mathrm{C}(15) & 113.34(13) \\ \mathrm{N}(2)-\mathrm{C}(12)-\mathrm{C}(13) & 130.04(14) \\ \mathrm{N}(2)-\mathrm{C}(12)-\mathrm{C}(11) & 113.71(14) \\ \mathrm{C}(13)-\mathrm{C}(12)-\mathrm{C}(11) & 116.14(13) \\ \mathrm{N}(1)-\mathrm{C}(13)-\mathrm{C}(8) & 110.76(13) \\ \mathrm{N}(1)-\mathrm{C}(13)-\mathrm{C}(12) & 128.89(13) \\ \mathrm{C}(8)-\mathrm{C}(13)-\mathrm{C}(12) & 120.27(14) \\ \mathrm{O}(1)-\mathrm{C}(14)-\mathrm{O}(2) & 121.90(16) \\ \mathrm{O}(1)-\mathrm{C}(14)-\mathrm{N}(2) & 126.48(16) \\ \mathrm{O}(2)-\mathrm{C}(14)-\mathrm{N}(2) & 111.62(14) \\ \mathrm{C}(11)-\mathrm{C}(15)-\mathrm{H}(15 \mathrm{~A}) & 109.5 \\ \mathrm{C}(11)-\mathrm{C}(15)-\mathrm{H}(15 \mathrm{~B}) & 109.5 \\ \mathrm{H}(15 \mathrm{~A})-\mathrm{C}(15)-\mathrm{H}(15 \mathrm{~B}) & 109.5 \\ \mathrm{C}(11)-\mathrm{C}(15)-\mathrm{H}(15 \mathrm{C}) & 109.5 \\ \mathrm{H}(15 \mathrm{~A})-\mathrm{C}(15)-\mathrm{H}(15 \mathrm{C}) & 109.5 \\ \mathrm{H}(15 \mathrm{~B})-\mathrm{C}(15)-\mathrm{H}(15 \mathrm{C}) & 109.5 \\ \mathrm{C}(2)-\mathrm{N}(1)-\mathrm{C}(13) & 107.22(12) \\ \mathrm{C}(2)-\mathrm{N}(1)-\mathrm{C}(1) & 126.01(14) \\ \mathrm{C}(13)-\mathrm{N}(1)-\mathrm{C}(1) & 126.62(13) \\ \mathrm{C}(12)-\mathrm{N}(2)-\mathrm{C}(14) & 105.57(13) \\ \mathrm{C}(14)-\mathrm{O}(2)-\mathrm{C}(11) & 107.90(12) \\ & \end{array}$

Symmetry transformations used to generate equivalent atoms:

Table S5. Anisotropic displacement parameters ( $\left.\mathrm{A}^{\wedge} 2 \times 10^{\wedge} 3\right)$ for ZYJ.

The anisotropic displacement factor exponent takes the form:

$-2 \mathrm{pi}^{\wedge} 2\left[\mathrm{~h}^{\wedge} 2 \mathrm{a}^{* \wedge} 2 \mathrm{U} 11+\ldots+2 \mathrm{~h} \mathrm{k} \mathrm{a} \mathrm{a}^{*} \mathrm{U} 12\right]$

$\begin{array}{llllll}\text { U11 } & \text { U22 } & \text { U33 } & \text { U23 } & \text { U13 } & \text { U12 }\end{array}$

$\begin{array}{llllrrr}\mathrm{C}(1) & 40(1) & 35(1) & 58(1) & 3(1) & 19(1) & 9(1) \\ \mathrm{C}(2) & 36(1) & 37(1) & 33(1) & -2(1) & 15(1) & -1(1) \\ \mathrm{C}(3) & 51(1) & 37(1) & 47(1) & -2(1) & 22(1) & -3(1) \\ \mathrm{C}(4) & 55(1) & 47(1) & 61(1) & -9(1) & 27(1) & -16(1)\end{array}$




$\begin{array}{llllrrr}\mathrm{C}(5) & 39(1) & 62(1) & 58(1) & -9(1) & 22(1) & -12(1) \\ \mathrm{C}(6) & 33(1) & 53(1) & 48(1) & -2(1) & 16(1) & -2(1) \\ \mathrm{C}(7) & 36(1) & 38(1) & 34(1) & 0(1) & 17(1) & 1(1) \\ \mathrm{C}(8) & 33(1) & 38(1) & 36(1) & -1(1) & 17(1) & 2(1) \\ \mathrm{C}(9) & 35(1) & 38(1) & 49(1) & 2(1) & 18(1) & 7(1) \\ \mathrm{C}(10) & 41(1) & 36(1) & 43(1) & 3(1) & 20(1) & 7(1) \\ \mathrm{C}(11) & 36(1) & 32(1) & 39(1) & -1(1) & 19(1) & -1(1) \\ \mathrm{C}(12) & 34(1) & 33(1) & 30(1) & 2(1) & 14(1) & 4(1) \\ \mathrm{C}(13) & 32(1) & 32(1) & 34(1) & 0(1) & 14(1) & 3(1) \\ \mathrm{C}(14) & 40(1) & 44(1) & 45(1) & 1(1) & 22(1) & 0(1) \\ \mathrm{C}(15) & 51(1) & 51(1) & 40(1) & -7(1) & 21(1) & 5(1) \\ \mathrm{N}(1) & 34(1) & 31(1) & 39(1) & -1(1) & 14(1) & 3(1) \\ \mathrm{N}(2) & 35(1) & 39(1) & 47(1) & -3(1) & 20(1) & 1(1) \\ \mathrm{O}(1) & 41(1) & 55(1) & 80(1) & 0(1) & 33(1) & -7(1) \\ \mathrm{O}(2) & 40(1) & 34(1) & 59(1) & -2(1) & 25(1) & -3(1)\end{array}$

Table S6. Hydrogen coordinates ( x 10^4) and isotropic displacement parameters $\left(\mathrm{A}^{\wedge} 2 \times 10^{\wedge} 3\right)$ for $\mathrm{ZYJ}$.

\begin{tabular}{|c|c|c|c|c|}
\hline & $\mathrm{x}$ & $\mathrm{y}$ & $\mathrm{z}$ & $\mathrm{U}(\mathrm{eq})$ \\
\hline $\mathrm{H}(1 \mathrm{~A})$ & 7747 & 2255 & 1955 & 67 \\
\hline $\mathrm{H}(1 \mathrm{~B})$ & 7172 & 1729 & 3006 & 67 \\
\hline $\mathrm{H}(1 \mathrm{C})$ & 6552 & 1551 & 1177 & 67 \\
\hline $\mathrm{H}(3)$ & 10573 & 2683 & 2961 & 54 \\
\hline $\mathrm{H}(4)$ & 13540 & 2854 & 3985 & 64 \\
\hline $\mathrm{H}(5)$ & 15423 & 1835 & 4667 & 64 \\
\hline $\mathrm{H}(6)$ & 14406 & 610 & 4342 & 55 \\
\hline $\mathrm{H}(9 \mathrm{~A})$ & 12238 & -662 & 4418 & 49 \\
\hline $\mathrm{H}(9 \mathrm{~B})$ & 11659 & -755 & 2569 & 49 \\
\hline $\mathrm{H}(10 \mathrm{~A})$ & 10006 & -1628 & 3078 & 47 \\
\hline $\mathrm{H}(10 \mathrm{~B})$ & 9638 & -1078 & 4233 & 47 \\
\hline $\mathrm{H}(15 \mathrm{~A})$ & 8141 & -1495 & 18 & 71 \\
\hline $\mathrm{H}(15 \mathrm{~B})$ & 9062 & -707 & 189 & 71 \\
\hline $\mathrm{H}(15 \mathrm{C})$ & 7029 & -754 & -612 & 71 \\
\hline
\end{tabular}


Table S7. Torsion angles [deg] for ZYJ.

\begin{tabular}{|c|c|}
\hline $\mathrm{N}(1)-\mathrm{C}(2)-\mathrm{C}(3)-\mathrm{C}(4)$ & $178.75(14)$ \\
\hline $\mathrm{C}(7)-\mathrm{C}(2)-\mathrm{C}(3)-\mathrm{C}(4)$ & $-1.0(2)$ \\
\hline $\mathrm{C}(2)-\mathrm{C}(3)-\mathrm{C}(4)-\mathrm{C}(5)$ & $0.6(3)$ \\
\hline $\mathrm{C}(3)-\mathrm{C}(4)-\mathrm{C}(5)-\mathrm{C}(6)$ & $0.1(3)$ \\
\hline$C(4)-C(5)-C(6)-C(7)$ & $-0.5(3)$ \\
\hline $\mathrm{C}(5)-\mathrm{C}(6)-\mathrm{C}(7)-\mathrm{C}(2)$ & $0.1(2)$ \\
\hline $\mathrm{C}(5)-\mathrm{C}(6)-\mathrm{C}(7)-\mathrm{C}(8)$ & $179.89(16)$ \\
\hline$N(1)-C(2)-C(7)-C(6)$ & $-179.13(13)$ \\
\hline $\mathrm{C}(3)-\mathrm{C}(2)-\mathrm{C}(7)-\mathrm{C}(6)$ & $0.6(2)$ \\
\hline $\mathrm{N}(1)-\mathrm{C}(2)-\mathrm{C}(7)-\mathrm{C}(8)$ & $1.03(15)$ \\
\hline $\mathrm{C}(3)-\mathrm{C}(2)-\mathrm{C}(7)-\mathrm{C}(8)$ & $-179.20(13)$ \\
\hline $\mathrm{C}(6)-\mathrm{C}(7)-\mathrm{C}(8)-\mathrm{C}(13)$ & $-179.75(16)$ \\
\hline$C(2)-C(7)-C(8)-C(13)$ & $0.05(15)$ \\
\hline $\mathrm{C}(6)-\mathrm{C}(7)-\mathrm{C}(8)-\mathrm{C}(9)$ & $3.0(3)$ \\
\hline $\mathrm{C}(2)-\mathrm{C}(7)-\mathrm{C}(8)-\mathrm{C}(9)$ & $-177.22(14)$ \\
\hline $\mathrm{C}(13)-\mathrm{C}(8)-\mathrm{C}(9)-\mathrm{C}(10)$ & $-12.02(19)$ \\
\hline C(7)-C(8)-C(9)-C(10) & $164.85(14)$ \\
\hline $\mathrm{C}(8)-\mathrm{C}(9)-\mathrm{C}(10)-\mathrm{C}(11)$ & 41.94(17) \\
\hline $\mathrm{C}(9)-\mathrm{C}(10)-\mathrm{C}(11)-\mathrm{O}(2)$ & $-170.90(11)$ \\
\hline $\mathrm{C}(9)-\mathrm{C}(10)-\mathrm{C}(11)-\mathrm{C}(12)$ & $-60.39(16)$ \\
\hline $\mathrm{C}(9)-\mathrm{C}(10)-\mathrm{C}(11)-\mathrm{C}(15)$ & $66.29(18)$ \\
\hline $\mathrm{O}(2)-\mathrm{C}(11)-\mathrm{C}(12)-\mathrm{N}(2)$ & $-9.05(16)$ \\
\hline $\mathrm{C}(10)-\mathrm{C}(11)-\mathrm{C}(12)-\mathrm{N}(2)$ & $-127.52(14)$ \\
\hline $\mathrm{C}(15)-\mathrm{C}(11)-\mathrm{C}(12)-\mathrm{N}(2)$ & $105.36(16)$ \\
\hline $\mathrm{O}(2)-\mathrm{C}(11)-\mathrm{C}(12)-\mathrm{C}(13)$ & $167.57(11)$ \\
\hline $\mathrm{C}(10)-\mathrm{C}(11)-\mathrm{C}(12)-\mathrm{C}(13)$ & $49.10(17)$ \\
\hline $\mathrm{C}(15)-\mathrm{C}(11)-\mathrm{C}(12)-\mathrm{C}(13)$ & $-78.01(17)$ \\
\hline $\mathrm{C}(7)-\mathrm{C}(8)-\mathrm{C}(13)-\mathrm{N}(1)$ & $-1.13(16)$ \\
\hline C(9)-C(8)-C(13)-N(1) & $176.40(13)$ \\
\hline $\mathrm{C}(7)-\mathrm{C}(8)-\mathrm{C}(13)-\mathrm{C}(12)$ & $-178.12(12)$ \\
\hline $\mathrm{C}(9)-\mathrm{C}(8)-\mathrm{C}(13)-\mathrm{C}(12)$ & $-0.6(2)$ \\
\hline $\mathrm{N}(2)-\mathrm{C}(12)-\mathrm{C}(13)-\mathrm{N}(1)$ & $-19.0(3)$ \\
\hline $\mathrm{C}(11)-\mathrm{C}(12)-\mathrm{C}(13)-\mathrm{N}(1)$ & $165.02(13)$ \\
\hline $\mathrm{N}(2)-\mathrm{C}(12)-\mathrm{C}(13)-\mathrm{C}(8)$ & $157.36(15)$ \\
\hline $\mathrm{C}(11)-\mathrm{C}(12)-\mathrm{C}(13)-\mathrm{C}(8)$ & $-18.60(19)$ \\
\hline $\mathrm{C}(3)-\mathrm{C}(2)-\mathrm{N}(1)-\mathrm{C}(13)$ & $178.55(15)$ \\
\hline $\mathrm{C}(7)-\mathrm{C}(2)-\mathrm{N}(1)-\mathrm{C}(13)$ & $-1.70(15)$ \\
\hline $\mathrm{C}(3)-\mathrm{C}(2)-\mathrm{N}(1)-\mathrm{C}(1)$ & $-5.7(2)$ \\
\hline $\mathrm{C}(7)-\mathrm{C}(2)-\mathrm{N}(1)-\mathrm{C}(1)$ & $174.00(13)$ \\
\hline $\mathrm{C}(8)-\mathrm{C}(13)-\mathrm{N}(1)-\mathrm{C}(2)$ & $1.78(15)$ \\
\hline
\end{tabular}




$\begin{array}{lr}\mathrm{C}(12)-\mathrm{C}(13)-\mathrm{N}(1)-\mathrm{C}(2) & 178.44(13) \\ \mathrm{C}(8)-\mathrm{C}(13)-\mathrm{N}(1)-\mathrm{C}(1) & -173.89(13) \\ \mathrm{C}(12)-\mathrm{C}(13)-\mathrm{N}(1)-\mathrm{C}(1) & 2.8(2) \\ \mathrm{C}(13)-\mathrm{C}(12)-\mathrm{N}(2)-\mathrm{C}(14) & -171.56(15) \\ \mathrm{C}(11)-\mathrm{C}(12)-\mathrm{N}(2)-\mathrm{C}(14) & 4.48(17) \\ \mathrm{O}(1)-\mathrm{C}(14)-\mathrm{N}(2)-\mathrm{C}(12) & -178.11(16) \\ \mathrm{O}(2)-\mathrm{C}(14)-\mathrm{N}(2)-\mathrm{C}(12) & 2.45(17) \\ \mathrm{O}(1)-\mathrm{C}(14)-\mathrm{O}(2)-\mathrm{C}(11) & 172.17(15) \\ \mathrm{N}(2)-\mathrm{C}(14)-\mathrm{O}(2)-\mathrm{C}(11) & -8.36(16) \\ \mathrm{C}(12)-\mathrm{C}(11)-\mathrm{O}(2)-\mathrm{C}(14) & 9.83(14) \\ \mathrm{C}(10)-\mathrm{C}(11)-\mathrm{O}(2)-\mathrm{C}(14) & 126.13(14) \\ \mathrm{C}(15)-\mathrm{C}(11)-\mathrm{O}(2)-\mathrm{C}(14) & -108.07(14) \\ \end{array}$

Symmetry transformations used to generate equivalent atoms:

Table S8. Hydrogen bonds for ZYJ [A and deg.].
D-H...A
$\mathrm{d}(\mathrm{D}-\mathrm{H})$
$\mathrm{d}(\mathrm{H} \ldots \mathrm{A}) \quad \mathrm{d}(\mathrm{D} \ldots \mathrm{A})$
$<$ (DHA)

2) Compound 10c
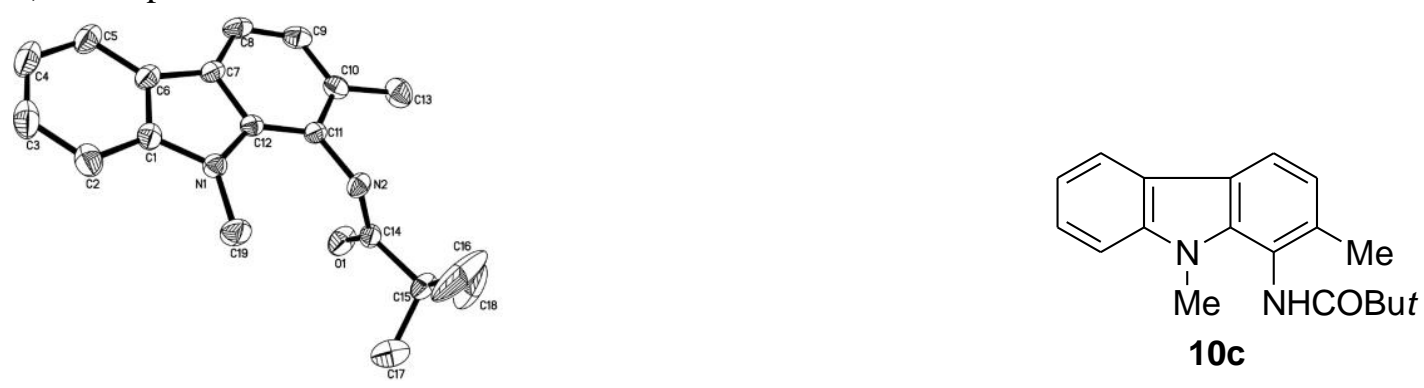

CCDC 1488849

Table S9. Crystal data and structure refinement for 10c.

Identification code

Empirical formula

Formula weight

Temperature 10c

C19 H22 N2 O

294.39

293(2) K 
Wavelength

Crystal system, space group

Unit cell dimensions

Volume

Z, Calculated density

Absorption coefficient

$\mathrm{F}(000)$

Crystal size

Theta range for data collection

Limiting indices

Reflections collected / unique

Completeness to theta $=27.48$

Max. and min. transmission

Refinement method

Data / restraints / parameters

Goodness-of-fit on $\mathrm{F}^{\wedge} 2$

Final $\mathrm{R}$ indices $[\mathrm{I}>2 \operatorname{sigma}(\mathrm{I})]$

$\mathrm{R}$ indices (all data)

Largest diff. peak and hole

\subsection{A}

Monoclinic, $\mathrm{P} 2(1) / \mathrm{c}$

$$
\begin{aligned}
& \mathrm{a}=13.832(3) \mathrm{A} \quad \text { alpha }=90 \mathrm{deg} . \\
& \mathrm{b}=10.231(2) \mathrm{A} \quad \text { beta }=111.27(3) \mathrm{deg} . \\
& \mathrm{c}=12.367(3) \mathrm{A} \quad \text { gamma }=90 \mathrm{deg} .
\end{aligned}
$$

$$
1630.9(6) \mathrm{A}^{\wedge} 3
$$

\section{4, $1.199 \mathrm{Mg} / \mathrm{m}^{\wedge} 3$}

$0.075 \mathrm{~mm}^{\wedge}-1$

632

$0.53 \times 0.45 \times 0.13 \mathrm{~mm}$

3.16 to $27.48 \mathrm{deg}$.

$-17<=\mathrm{h}<=17,-12<=\mathrm{k}<=13,-15<=\mathrm{l}<=14$

$15327 / 3677[\mathrm{R}(\mathrm{int})=0.0369]$

$98.5 \%$

0.9901 and 0.9613

Full-matrix least-squares on $\mathrm{F}^{\wedge} 2$

3677 / 0 / 208

1.097

$\mathrm{R} 1=0.0628, \mathrm{wR} 2=0.1722$

$\mathrm{R} 1=0.0879, \mathrm{wR} 2=0.1858$

0.375 and -0.299 e. $\mathrm{A}^{\wedge}-3$ 
Table S10. Atomic coordinates ( $\times 10^{\wedge} 4$ ) and equivalent isotropic displacement parameters $\left(\mathrm{A}^{\wedge} 2 \times 10^{\wedge} 3\right)$ for gaowei.

$\mathrm{U}(\mathrm{eq})$ is defined as one third of the trace of the orthogonalized

Uij tensor.

\begin{tabular}{lrrrr}
\hline & & & & \\
& $\mathrm{x}$ & $\mathrm{y}$ & $\mathrm{Z}$ & $\mathrm{U}(\mathrm{eq})$ \\
& & & & \\
\hline & & & & \\
$\mathrm{C}(1)$ & $10856(2)$ & $8073(2)$ & $471(2)$ & $41(1)$ \\
$\mathrm{C}(2)$ & $11469(2)$ & $7407(2)$ & $-27(2)$ & $57(1)$ \\
$\mathrm{C}(3)$ & $12525(2)$ & $7602(3)$ & $455(3)$ & $70(1)$ \\
$\mathrm{C}(4)$ & $12971(2)$ & $8428(3)$ & $1393(3)$ & $71(1)$ \\
$\mathrm{C}(5)$ & $12370(2)$ & $9087(2)$ & $1890(2)$ & $58(1)$ \\
$\mathrm{C}(6)$ & $11298(2)$ & $8906(2)$ & $1428(2)$ & $43(1)$ \\
$\mathrm{C}(7)$ & $10453(2)$ & $9407(2)$ & $1728(2)$ & $39(1)$ \\
$\mathrm{C}(8)$ & $10403(2)$ & $10274(2)$ & $2570(2)$ & $51(1)$ \\
$\mathrm{C}(9)$ & $9458(2)$ & $10551(2)$ & $2634(2)$ & $53(1)$ \\
$\mathrm{C}(10)$ & $8541(2)$ & $10005(2)$ & $1866(2)$ & $44(1)$ \\
$\mathrm{C}(11)$ & $8576(1)$ & $9145(2)$ & $1010(2)$ & $36(1)$ \\
$\mathrm{C}(12)$ & $9537(1)$ & $8844(2)$ & $941(2)$ & $34(1)$ \\
$\mathrm{C}(13)$ & $7528(2)$ & $10374(3)$ & $1972(2)$ & $65(1)$ \\
$\mathrm{C}(14)$ & $7062(2)$ & $7688(2)$ & $407(2)$ & $39(1)$ \\
$\mathrm{C}(15)$ & $6023(2)$ & $7352(2)$ & $-550(2)$ & $50(1)$ \\
$\mathrm{C}(16)$ & $5790(3)$ & $8124(6)$ & $-1648(3)$ & $166(3)$ \\
$\mathrm{C}(17)$ & $6062(3)$ & $5912(4)$ & $-832(5)$ & $148(2)$ \\
$\mathrm{C}(18)$ & $5193(3)$ & $7533(5)$ & $-68(4)$ & $128(2)$ \\
$\mathrm{C}(19)$ & $9104(2)$ & $7241(2)$ & $-749(2)$ & $58(1)$ \\
$\mathrm{N}(1)$ & $9792(1)$ & $8041(2)$ & $178(1)$ & $38(1)$ \\
$\mathrm{N}(2)$ & $7630(1)$ & $8647(2)$ & $183(2)$ & $40(1)$ \\
$\mathrm{O}(1)$ & $7360(1)$ & $7109(2)$ & $1330(2)$ & $65(1)$ \\
& & & & \\
\hline
\end{tabular}

Table S11. Bond lengths [A] and angles [deg] for gaowei.

$\begin{array}{ll}\mathrm{C}(1)-\mathrm{N}(1) & 1.382(3) \\ \mathrm{C}(1)-\mathrm{C}(2) & 1.393(3) \\ \mathrm{C}(1)-\mathrm{C}(6) & 1.405(3) \\ \mathrm{C}(2)-\mathrm{C}(3) & 1.378(4) \\ \mathrm{C}(2)-\mathrm{H}(2) & 0.9300\end{array}$




\begin{tabular}{|c|c|}
\hline$C(3)-C(4)$ & $1.386(4)$ \\
\hline $\mathrm{C}(3)-\mathrm{H}(3)$ & 0.9300 \\
\hline$C(4)-C(5)$ & $1.377(4)$ \\
\hline $\mathrm{C}(4)-\mathrm{H}(4)$ & 0.9300 \\
\hline$C(5)-C(6)$ & $1.395(3)$ \\
\hline $\mathrm{C}(5)-\mathrm{H}(5)$ & 0.9300 \\
\hline$C(6)-C(7)$ & $1.441(3)$ \\
\hline $\mathrm{C}(7)-\mathrm{C}(8)$ & $1.389(3)$ \\
\hline$C(7)-C(12)$ & $1.411(3)$ \\
\hline $\mathrm{C}(8)-\mathrm{C}(9)$ & $1.367(3)$ \\
\hline $\mathrm{C}(8)-\mathrm{H}(8)$ & 0.9300 \\
\hline C(9)-C(10) & $1.397(3)$ \\
\hline $\mathrm{C}(9)-\mathrm{H}(9)$ & 0.9300 \\
\hline$C(10)-C(11)$ & $1.391(3)$ \\
\hline$C(10)-C(13)$ & $1.502(3)$ \\
\hline $\mathrm{C}(11)-\mathrm{C}(12)$ & $1.398(3)$ \\
\hline $\mathrm{C}(11)-\mathrm{N}(2)$ & $1.431(2)$ \\
\hline $\mathrm{C}(12)-\mathrm{N}(1)$ & $1.390(2)$ \\
\hline $\mathrm{C}(13)-\mathrm{H}(13 \mathrm{~A})$ & 0.9600 \\
\hline $\mathrm{C}(13)-\mathrm{H}(13 \mathrm{~B})$ & 0.9600 \\
\hline $\mathrm{C}(13)-\mathrm{H}(13 \mathrm{C})$ & 0.9600 \\
\hline $\mathrm{C}(14)-\mathrm{O}(1)$ & $1.217(2)$ \\
\hline $\mathrm{C}(14)-\mathrm{N}(2)$ & $1.347(2)$ \\
\hline$C(14)-C(15)$ & $1.533(3)$ \\
\hline $\mathrm{C}(15)-\mathrm{C}(18)$ & $1.485(4)$ \\
\hline$C(15)-C(16)$ & $1.502(4)$ \\
\hline C(15)-C(17) & $1.519(4)$ \\
\hline$C(16)-H(16 A)$ & 0.9600 \\
\hline $\mathrm{C}(16)-\mathrm{H}(16 \mathrm{~B})$ & 0.9600 \\
\hline $\mathrm{C}(16)-\mathrm{H}(16 \mathrm{C})$ & 0.9600 \\
\hline $\mathrm{C}(17)-\mathrm{H}(17 \mathrm{~A})$ & 0.9600 \\
\hline $\mathrm{C}(17)-\mathrm{H}(17 \mathrm{~B})$ & 0.9600 \\
\hline $\mathrm{C}(17)-\mathrm{H}(17 \mathrm{C})$ & 0.9600 \\
\hline $\mathrm{C}(18)-\mathrm{H}(18 \mathrm{~A})$ & 0.9600 \\
\hline $\mathrm{C}(18)-\mathrm{H}(18 \mathrm{~B})$ & 0.9600 \\
\hline $\mathrm{C}(18)-\mathrm{H}(18 \mathrm{C})$ & 0.9600 \\
\hline $\mathrm{C}(19)-\mathrm{N}(1)$ & $1.448(3)$ \\
\hline $\mathrm{C}(19)-\mathrm{H}(19 \mathrm{~A})$ & 0.9600 \\
\hline $\mathrm{C}(19)-\mathrm{H}(19 \mathrm{~B})$ & 0.9600 \\
\hline C(19)-H(19C) & 0.9600 \\
\hline $\mathrm{N}(2)-\mathrm{H}(1)$ & $0.83(3)$ \\
\hline $\mathrm{N}(1)-\mathrm{C}(1)-\mathrm{C}(2)$ & $129.2(2)$ \\
\hline $\mathrm{N}(1)-\mathrm{C}(1)-\mathrm{C}(6)$ & $109.37(17)$ \\
\hline
\end{tabular}




\begin{tabular}{|c|c|}
\hline$C(2)-C(1)-C(6)$ & $121.4(2)$ \\
\hline $\mathrm{C}(3)-\mathrm{C}(2)-\mathrm{C}(1)$ & $117.2(3)$ \\
\hline $\mathrm{C}(3)-\mathrm{C}(2)-\mathrm{H}(2)$ & 121.4 \\
\hline $\mathrm{C}(1)-\mathrm{C}(2)-\mathrm{H}(2)$ & 121.4 \\
\hline $\mathrm{C}(2)-\mathrm{C}(3)-\mathrm{C}(4)$ & $122.0(2)$ \\
\hline $\mathrm{C}(2)-\mathrm{C}(3)-\mathrm{H}(3)$ & 119.0 \\
\hline $\mathrm{C}(4)-\mathrm{C}(3)-\mathrm{H}(3)$ & 119.0 \\
\hline $\mathrm{C}(5)-\mathrm{C}(4)-\mathrm{C}(3)$ & $121.0(2)$ \\
\hline $\mathrm{C}(5)-\mathrm{C}(4)-\mathrm{H}(4)$ & 119.5 \\
\hline $\mathrm{C}(3)-\mathrm{C}(4)-\mathrm{H}(4)$ & 119.5 \\
\hline$C(4)-C(5)-C(6)$ & $118.4(3)$ \\
\hline $\mathrm{C}(4)-\mathrm{C}(5)-\mathrm{H}(5)$ & 120.8 \\
\hline $\mathrm{C}(6)-\mathrm{C}(5)-\mathrm{H}(5)$ & 120.8 \\
\hline$C(5)-C(6)-C(1)$ & $119.9(2)$ \\
\hline$C(5)-C(6)-C(7)$ & $133.4(2)$ \\
\hline $\mathrm{C}(1)-\mathrm{C}(6)-\mathrm{C}(7)$ & $106.67(17)$ \\
\hline $\mathrm{C}(8)-\mathrm{C}(7)-\mathrm{C}(12)$ & $120.07(18)$ \\
\hline $\mathrm{C}(8)-\mathrm{C}(7)-\mathrm{C}(6)$ & $133.29(19)$ \\
\hline $\mathrm{C}(12)-\mathrm{C}(7)-\mathrm{C}(6)$ & $106.64(17)$ \\
\hline C(9)-C(8)-C(7) & 119.01(19) \\
\hline $\mathrm{C}(9)-\mathrm{C}(8)-\mathrm{H}(8)$ & 120.5 \\
\hline $\mathrm{C}(7)-\mathrm{C}(8)-\mathrm{H}(8)$ & 120.5 \\
\hline C(8)-C(9)-C(10) & $121.96(19)$ \\
\hline C(8)-C(9)-H(9) & 119.0 \\
\hline $\mathrm{C}(10)-\mathrm{C}(9)-\mathrm{H}(9)$ & 119.0 \\
\hline C(11)-C(10)-C(9) & $119.81(19)$ \\
\hline $\mathrm{C}(11)-\mathrm{C}(10)-\mathrm{C}(13)$ & $121.1(2)$ \\
\hline C(9)-C(10)-C(13) & $119.1(2)$ \\
\hline $\mathrm{C}(10)-\mathrm{C}(11)-\mathrm{C}(12)$ & $118.84(18)$ \\
\hline $\mathrm{C}(10)-\mathrm{C}(11)-\mathrm{N}(2)$ & $119.62(17)$ \\
\hline $\mathrm{C}(12)-\mathrm{C}(11)-\mathrm{N}(2)$ & $121.44(16)$ \\
\hline $\mathrm{N}(1)-\mathrm{C}(12)-\mathrm{C}(11)$ & $130.80(17)$ \\
\hline $\mathrm{N}(1)-\mathrm{C}(12)-\mathrm{C}(7)$ & $108.89(16)$ \\
\hline $\mathrm{C}(11)-\mathrm{C}(12)-\mathrm{C}(7)$ & $120.31(17)$ \\
\hline $\mathrm{C}(10)-\mathrm{C}(13)-\mathrm{H}(13 \mathrm{~A})$ & 109.5 \\
\hline C(10)-C(13)-H(13B) & 109.5 \\
\hline $\mathrm{H}(13 \mathrm{~A})-\mathrm{C}(13)-\mathrm{H}(13 \mathrm{~B})$ & 109.5 \\
\hline $\mathrm{C}(10)-\mathrm{C}(13)-\mathrm{H}(13 \mathrm{C})$ & 109.5 \\
\hline $\mathrm{H}(13 \mathrm{~A})-\mathrm{C}(13)-\mathrm{H}(13 \mathrm{C})$ & 109.5 \\
\hline $\mathrm{H}(13 \mathrm{~B})-\mathrm{C}(13)-\mathrm{H}(13 \mathrm{C})$ & 109.5 \\
\hline $\mathrm{O}(1)-\mathrm{C}(14)-\mathrm{N}(2)$ & $121.55(19)$ \\
\hline $\mathrm{O}(1)-\mathrm{C}(14)-\mathrm{C}(15)$ & $121.20(18)$ \\
\hline $\mathrm{N}(2)-\mathrm{C}(14)-\mathrm{C}(15)$ & $117.26(17)$ \\
\hline$C(18)-C(15)-C(16)$ & $110.7(3)$ \\
\hline
\end{tabular}




$\begin{array}{lc}\mathrm{C}(18)-\mathrm{C}(15)-\mathrm{C}(17) & 108.0(3) \\ \mathrm{C}(16)-\mathrm{C}(15)-\mathrm{C}(17) & 108.4(4) \\ \mathrm{C}(18)-\mathrm{C}(15)-\mathrm{C}(14) & 107.9(2) \\ \mathrm{C}(16)-\mathrm{C}(15)-\mathrm{C}(14) & 114.4(2) \\ \mathrm{C}(17)-\mathrm{C}(15)-\mathrm{C}(14) & 107.1(2) \\ \mathrm{C}(15)-\mathrm{C}(16)-\mathrm{H}(16 \mathrm{~A}) & 109.5 \\ \mathrm{C}(15)-\mathrm{C}(16)-\mathrm{H}(16 \mathrm{~B}) & 109.5 \\ \mathrm{H}(16 \mathrm{~A})-\mathrm{C}(16)-\mathrm{H}(16 \mathrm{~B}) & 109.5 \\ \mathrm{C}(15)-\mathrm{C}(16)-\mathrm{H}(16 \mathrm{C}) & 109.5 \\ \mathrm{H}(16 \mathrm{~A})-\mathrm{C}(16)-\mathrm{H}(16 \mathrm{C}) & 109.5 \\ \mathrm{H}(16 \mathrm{~B})-\mathrm{C}(16)-\mathrm{H}(16 \mathrm{C}) & 109.5 \\ \mathrm{C}(15)-\mathrm{C}(17)-\mathrm{H}(17 \mathrm{~A}) & 109.5 \\ \mathrm{C}(15)-\mathrm{C}(17)-\mathrm{H}(17 \mathrm{~B}) & 109.5 \\ \mathrm{H}(17 \mathrm{~A})-\mathrm{C}(17)-\mathrm{H}(17 \mathrm{~B}) & 109.5 \\ \mathrm{C}(15)-\mathrm{C}(17)-\mathrm{H}(17 \mathrm{C}) & 109.5 \\ \mathrm{H}(17 \mathrm{~A})-\mathrm{C}(17)-\mathrm{H}(17 \mathrm{C}) & 109.5 \\ \mathrm{H}(17 \mathrm{~B})-\mathrm{C}(17)-\mathrm{H}(17 \mathrm{C}) & 109.5 \\ \mathrm{C}(15)-\mathrm{C}(18)-\mathrm{H}(18 \mathrm{~A}) & 109.5 \\ \mathrm{C}(15)-\mathrm{C}(18)-\mathrm{H}(18 \mathrm{~B}) & 109.5 \\ \mathrm{H}(18 \mathrm{~A})-\mathrm{C}(18)-\mathrm{H}(18 \mathrm{~B}) & 109.5 \\ \mathrm{C}(15)-\mathrm{C}(18)-\mathrm{H}(18 \mathrm{C}) & 109.5 \\ \mathrm{H}(18 \mathrm{~A})-\mathrm{C}(18)-\mathrm{H}(18 \mathrm{C}) & 109.5 \\ \mathrm{H}(18 \mathrm{~B})-\mathrm{C}(18)-\mathrm{H}(18 \mathrm{C}) & 109.5 \\ \text { N(1)-C(19)-H(19A) } & 109.5 \\ \text { N(1)-C(19)-H(19B)} & 109.5 \\ \mathrm{H}(19 \mathrm{~A})-\mathrm{C}(19)-\mathrm{H}(19 \mathrm{~B}) & 109.5 \\ \mathrm{~N}(1)-\mathrm{C}(19)-\mathrm{H}(19 \mathrm{C}) & 109.5 \\ \mathrm{H}(19 \mathrm{~A})-\mathrm{C}(19)-\mathrm{H}(19 \mathrm{C}) & 109.5 \\ \mathrm{H}(19 \mathrm{~B})-\mathrm{C}(19)-\mathrm{H}(19 \mathrm{C}) & 109.5 \\ \mathrm{C}(1)-\mathrm{N}(1)-\mathrm{C}(12) & 108.42(16) \\ \mathrm{C}(1)-\mathrm{N}(1)-\mathrm{C}(19) & 123.58(17) \\ \mathrm{C}(12)-\mathrm{N}(1)-\mathrm{C}(19) & 127.95(17) \\ \mathrm{C}(14)-\mathrm{N}(2)-\mathrm{C}(11) & 124.23(17) \\ \mathrm{C}(14)-\mathrm{N}(2)-\mathrm{H}(1) & 118.4(18) \\ \mathrm{C}(11)-\mathrm{N}(2)-\mathrm{H}(1) & 117.1(18) \\ & \\ & \end{array}$

Symmetry transformations used to generate equivalent atoms:

Table S12. Anisotropic displacement parameters $\left(\mathrm{A}^{\wedge} 2 \times 10^{\wedge} 3\right)$ for gaowei.

The anisotropic displacement factor exponent takes the form:

$-2 \mathrm{pi}^{\wedge} 2\left[\mathrm{~h}^{\wedge} 2 \mathrm{a}^{* \wedge} 2 \mathrm{U} 11+\ldots+2 \mathrm{~h} \mathrm{k} \mathrm{a*} \mathrm{b}^{*} \mathrm{U} 12\right]$ 


\begin{tabular}{lrrrrrr}
\hline & & & & & & \\
$\mathrm{C}(1)$ & $40(1)$ & $40(1)$ & $45(1)$ & $13(1)$ & $16(1)$ & $3(1)$ \\
$\mathrm{C}(2)$ & $59(2)$ & $52(1)$ & $67(2)$ & $11(1)$ & $32(1)$ & $8(1)$ \\
$\mathrm{C}(3)$ & $55(2)$ & $68(2)$ & $99(2)$ & $32(2)$ & $45(2)$ & $20(1)$ \\
$\mathrm{C}(4)$ & $36(1)$ & $75(2)$ & $97(2)$ & $36(2)$ & $20(1)$ & $5(1)$ \\
$\mathrm{C}(5)$ & $38(1)$ & $59(1)$ & $66(1)$ & $23(1)$ & $7(1)$ & $-5(1)$ \\
$\mathrm{C}(6)$ & $37(1)$ & $41(1)$ & $45(1)$ & $14(1)$ & $7(1)$ & $-4(1)$ \\
$\mathrm{C}(7)$ & $37(1)$ & $37(1)$ & $37(1)$ & $5(1)$ & $6(1)$ & $-7(1)$ \\
$\mathrm{C}(8)$ & $56(1)$ & $45(1)$ & $41(1)$ & $-5(1)$ & $5(1)$ & $-16(1)$ \\
$\mathrm{C}(9)$ & $69(2)$ & $43(1)$ & $44(1)$ & $-11(1)$ & $18(1)$ & $-8(1)$ \\
$\mathrm{C}(10)$ & $53(1)$ & $38(1)$ & $44(1)$ & $2(1)$ & $19(1)$ & $0(1)$ \\
$\mathrm{C}(11)$ & $36(1)$ & $33(1)$ & $34(1)$ & $4(1)$ & $8(1)$ & $-2(1)$ \\
$\mathrm{C}(12)$ & $38(1)$ & $31(1)$ & $31(1)$ & $3(1)$ & $8(1)$ & $-2(1)$ \\
$\mathrm{C}(13)$ & $69(2)$ & $65(2)$ & $72(2)$ & $-4(1)$ & $39(1)$ & $9(1)$ \\
$\mathrm{C}(14)$ & $34(1)$ & $40(1)$ & $43(1)$ & $4(1)$ & $13(1)$ & $1(1)$ \\
$\mathrm{C}(15)$ & $36(1)$ & $60(1)$ & $50(1)$ & $1(1)$ & $10(1)$ & $-11(1)$ \\
$\mathrm{C}(16)$ & $113(3)$ & $243(6)$ & $75(2)$ & $66(3)$ & $-45(2)$ & $-111(4)$ \\
$\mathrm{C}(17)$ & $97(3)$ & $104(3)$ & $195(5)$ & $-75(3)$ & $-4(3)$ & $-14(2)$ \\
$\mathrm{C}(18)$ & $45(2)$ & $224(5)$ & $112(3)$ & $-29(3)$ & $24(2)$ & $-4(2)$ \\
$\mathrm{C}(19)$ & $54(1)$ & $64(2)$ & $52(1)$ & $-24(1)$ & $15(1)$ & $-9(1)$ \\
$\mathrm{N}(1)$ & $37(1)$ & $39(1)$ & $36(1)$ & $-1(1)$ & $11(1)$ & $-1(1)$ \\
$\mathrm{N}(2)$ & $34(1)$ & $47(1)$ & $36(1)$ & $7(1)$ & $7(1)$ & $-4(1)$ \\
$\mathrm{O}(1)$ & $52(1)$ & $67(1)$ & $62(1)$ & $28(1)$ & $4(1)$ & $-9(1)$ \\
& & & & & & \\
\hline
\end{tabular}

Table S13. Hydrogen coordinates ( $\left.\times 10^{\wedge} 4\right)$ and isotropic displacement parameters $\left(\mathrm{A}^{\wedge} 2 \times 10^{\wedge} 3\right)$ for gaowei.

\begin{tabular}{lcccc}
\hline & & & & \\
& $x$ & $y$ & $z$ & $U(e q)$ \\
\hline & & & & \\
$H(2)$ & 11177 & 6853 & -658 & 68 \\
$\mathrm{H}(3)$ & 12952 & 7167 & 140 & 83 \\
$\mathrm{H}(4)$ & 13687 & 8538 & 1691 & 85 \\
$\mathrm{H}(5)$ & 12672 & 9640 & 2520 & 69 \\
$\mathrm{H}(8)$ & 11003 & 10660 & 3084 & 61 \\
$\mathrm{H}(9)$ & 9425 & 11121 & 3206 & 63 \\
$\mathrm{H}(13 \mathrm{~A})$ & 7062 & 10702 & 1242 & 97 \\
& & & &
\end{tabular}




\begin{tabular}{llrrr}
$\mathrm{H}(13 \mathrm{~B})$ & 7646 & 11036 & 2556 & 97 \\
$\mathrm{H}(13 \mathrm{C})$ & 7227 & 9618 & 2188 & 97 \\
$\mathrm{H}(16 \mathrm{~A})$ & 5131 & 7857 & -2203 & 249 \\
$\mathrm{H}(16 \mathrm{~B})$ & 6322 & 7970 & -1961 & 249 \\
$\mathrm{H}(16 \mathrm{C})$ & 5768 & 9038 & -1484 & 249 \\
$\mathrm{H}(17 \mathrm{~A})$ & 5379 & 5619 & -1304 & 222 \\
$\mathrm{H}(17 \mathrm{~B})$ & 6306 & 5416 & -125 & 222 \\
$\mathrm{H}(17 \mathrm{C})$ & 6524 & 5790 & -1245 & 222 \\
$\mathrm{H}(18 \mathrm{~A})$ & 5137 & 8443 & 90 & 192 \\
$\mathrm{H}(18 \mathrm{~B})$ & 5361 & 7042 & 639 & 192 \\
$\mathrm{H}(18 \mathrm{C})$ & 4545 & 7230 & -619 & 192 \\
$\mathrm{H}(19 \mathrm{~A})$ & 9498 & 6792 & -1133 & 87 \\
$\mathrm{H}(19 \mathrm{~B})$ & 8594 & 7787 & -1296 & 87 \\
$\mathrm{H}(19 \mathrm{C})$ & 8764 & 6614 & -431 & 87 \\
$\mathrm{H}(1)$ & $7390(20)$ & $9020(30)$ & $-460(20)$ & $64(8)$ \\
& & & & \\
\hline
\end{tabular}

Table S14. Torsion angles [deg] for gaowei. 